\title{
Iron-Catalyzed Direct Julia-type Olefination of Alcohols
}

Vinod G. Landge, ${ }^{\dagger}$ Reshma Babu, ${ }^{\dagger}$ Vinita Yadav,, Murugan Subaramanian, ${ }^{\dagger}$ Virendrakumar ${\text { Gupta, }{ }^{\Upsilon^{*}} \text { and Ekambaram Balaraman }}^{{ }^{* *}}$

'Department of Chemistry, Indian Institute of Science Education and Research (IISER) Tirupati, Tirupati - 517507, India. E-mail: eb.raman@iisertirupati.ac.in

Organic Chemistry Division, Dr. Homi Bhabha Road, CSIR-National Chemical Laboratory (CSIR-NCL), Pune - 411008, India.

"Polymer Synthesis \& Catalysis, Reliance Research \& Development Centre, Reliance Industries Limited, Ghansoli 400701, Navi Mumbai, India. Email: virendrakumar.gupta@ril.com

\section{Contents}

1. Mechanistic studies

S2-S9

2. Copy of NMR Spectra

S10-S94 


\section{MECHANISTIC STUDIES}

\section{Unsuccessful substrates:}

The following alcohols are failed to yield the desired olefin under our Fe-catalyzed conditions.
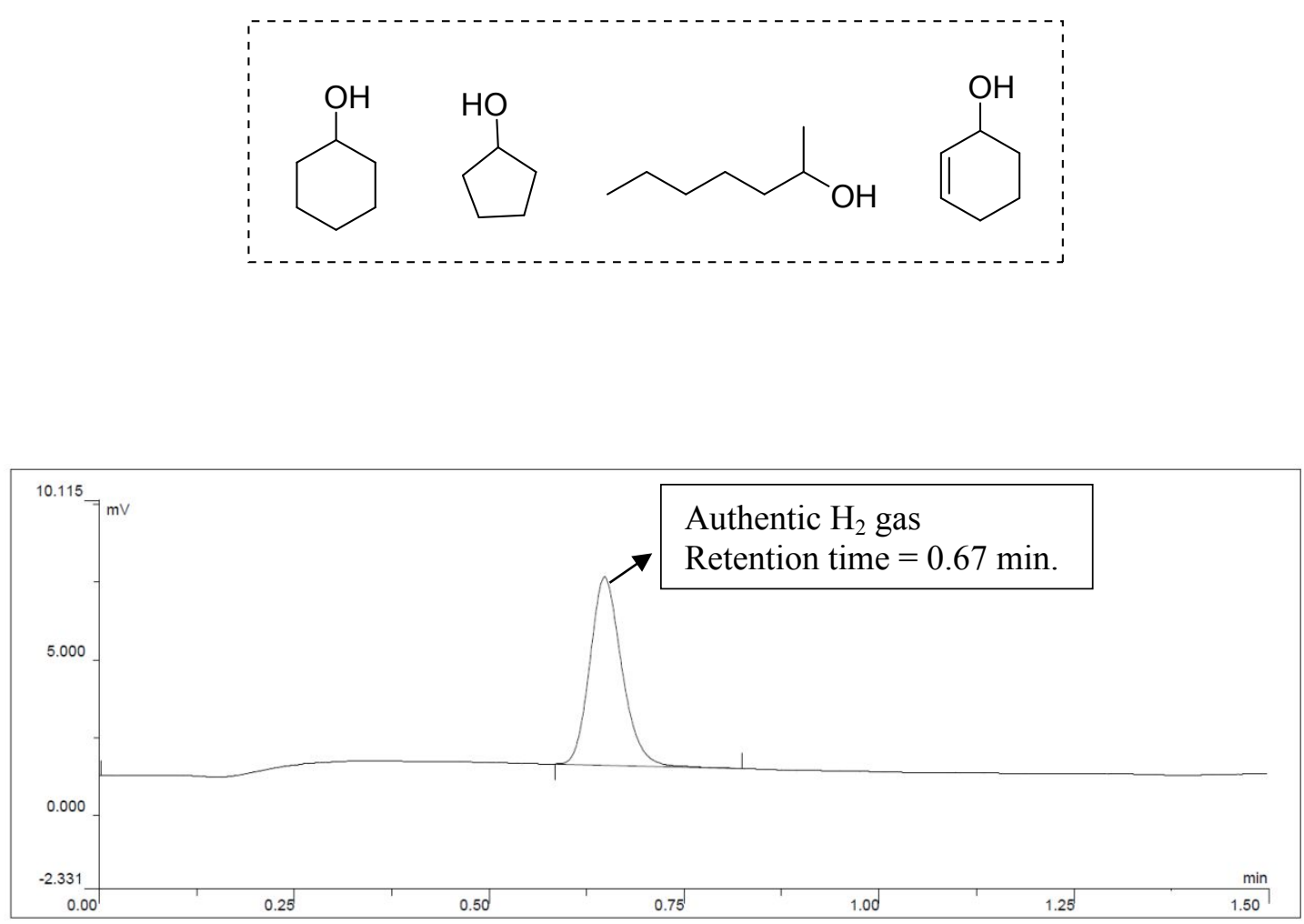

Figure S1A. GC chromatogram of authentic hydrogen gas.

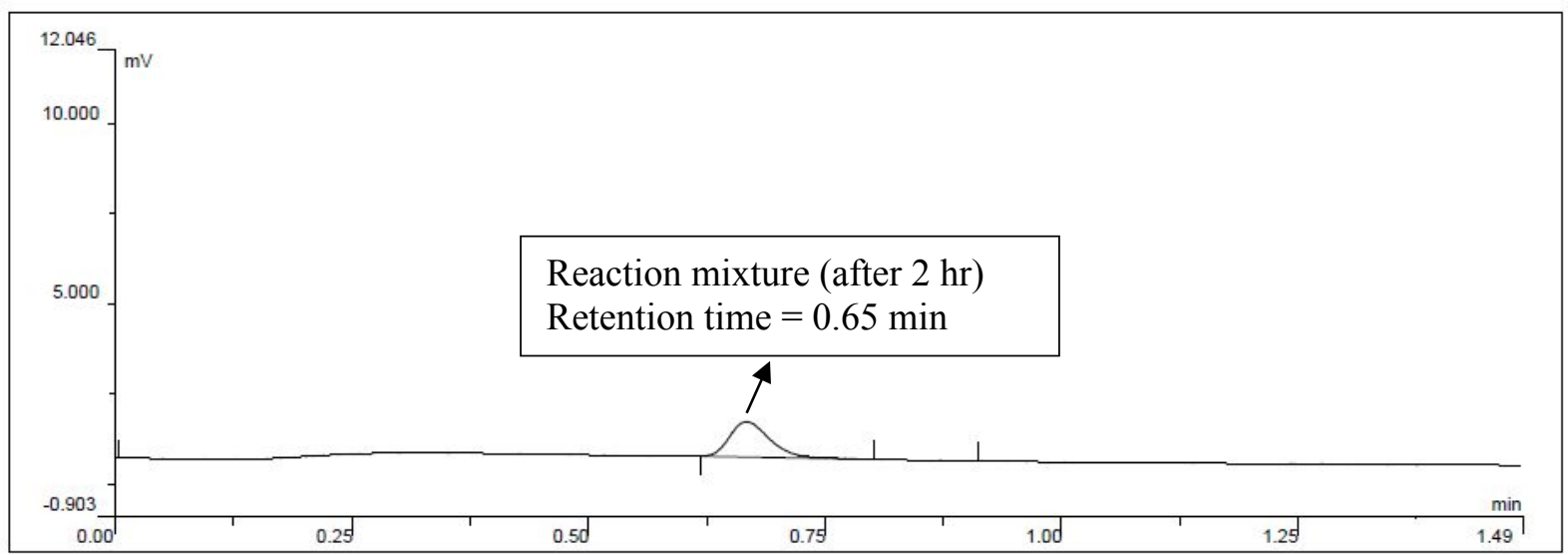

Figure S1B. GC chromatogram of gaseous sample of reaction mixture (after $2 \mathrm{hr}$ ). 

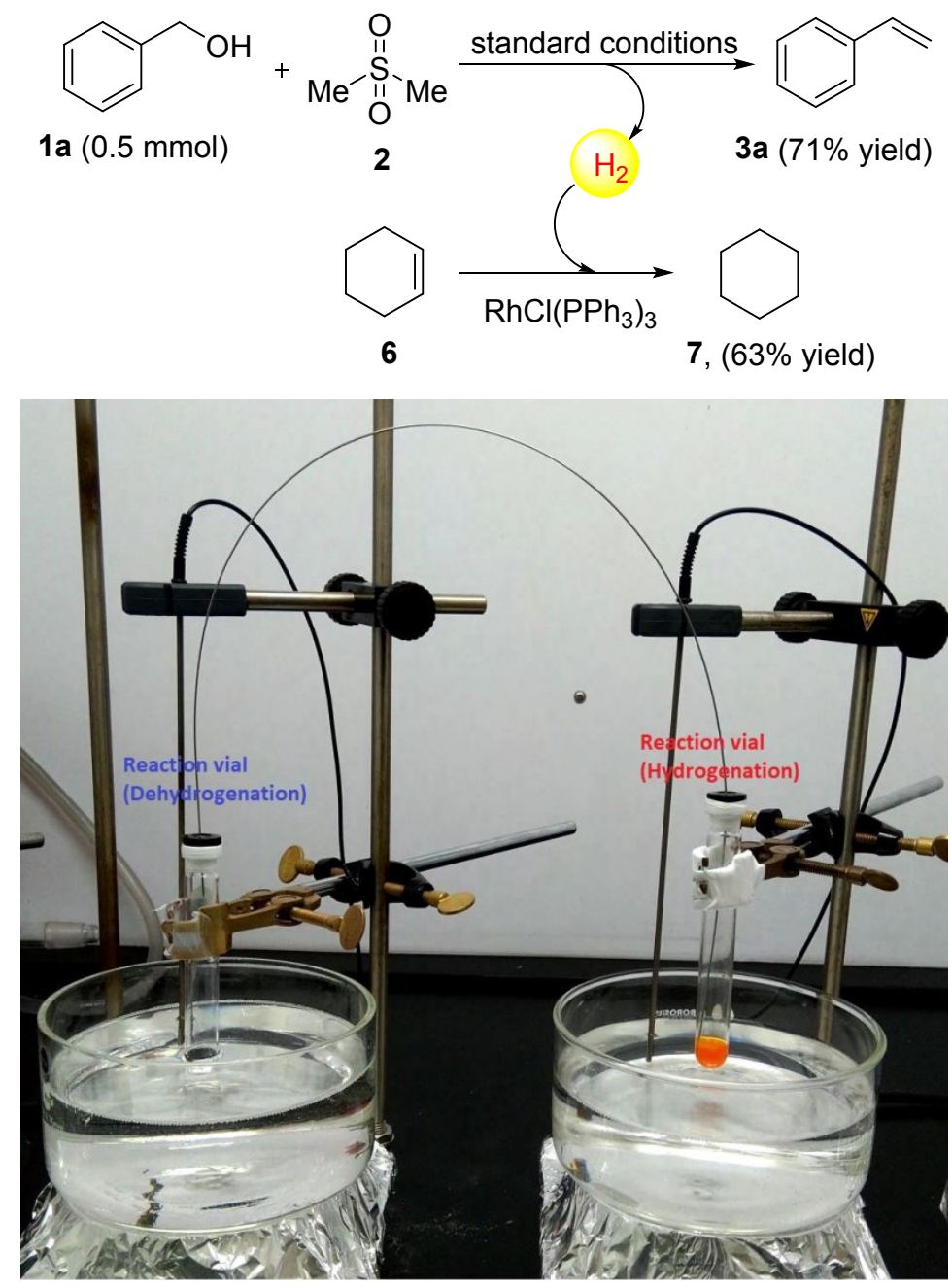

Figure S2. A picture of the apparatus used for the reaction. 


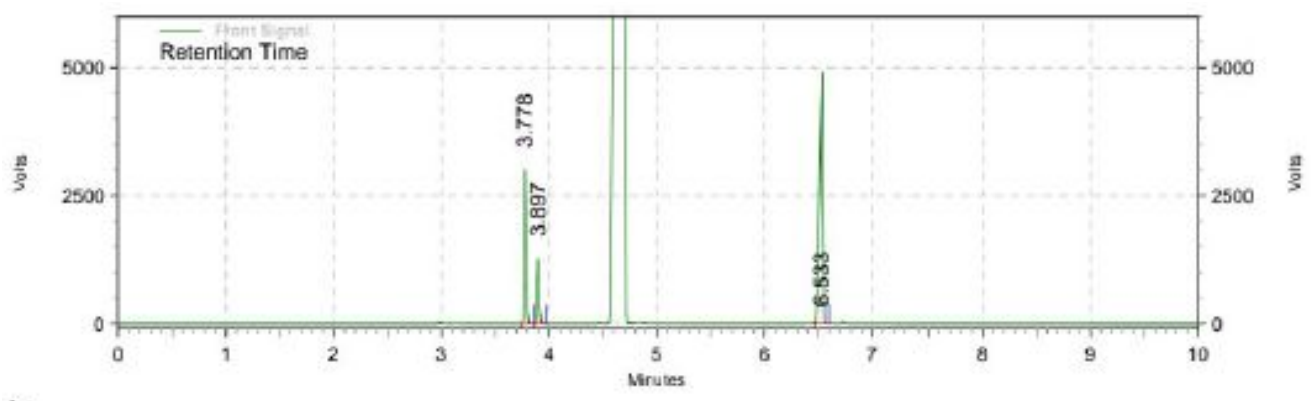

is

$\begin{aligned} & \text { Front Signal } \\
& \text { Results } \\
& \text { Retention Time }\end{aligned}$
\begin{tabular}{rrrrrrr} 
\\
3.778 & 30205498 & 25.28 & Height & Height \% & \\
\hline 3.897 & 13420568 & 11.23 & 22989272 & 32.65 & Cyclohexane \\
6.533 & 75845235 & 63.48 & 9649840 & 13.70 & Cyclohexene \\
& & 37773780 & 53.65 & m-xylene (Int.std) \\
\hline Totals & 119471301 & 100.00 & 70412892 & 100.00 \\
\hline
\end{tabular}
GC Condition:
Column $\quad:$ HP- $5(30 \mathrm{M} \mathrm{X} 0.32 \mathrm{~mm}$ X0.25um ID)
Carrier gas $\quad$ : Nitrogen, Flow : ( $1 \mathrm{ml} / \mathrm{min})$ (split ratio: 100:1)
Injection volume : $0.5 \mathrm{uL}$
Detector : FID, temp : $300^{\circ} \mathrm{C}$
Injector temp. $\quad: 250^{\circ} \mathrm{C}$
Temp program $\quad: 50^{\circ} \mathrm{C}(2 \mathrm{~min}$. hold $)-20^{\circ} \mathrm{C} / \mathrm{min}-280^{\circ} \mathrm{C}(0 \mathrm{~min}$.hold $)$

Figure S3A. GC traces for the reaction mixture (with internal standard).

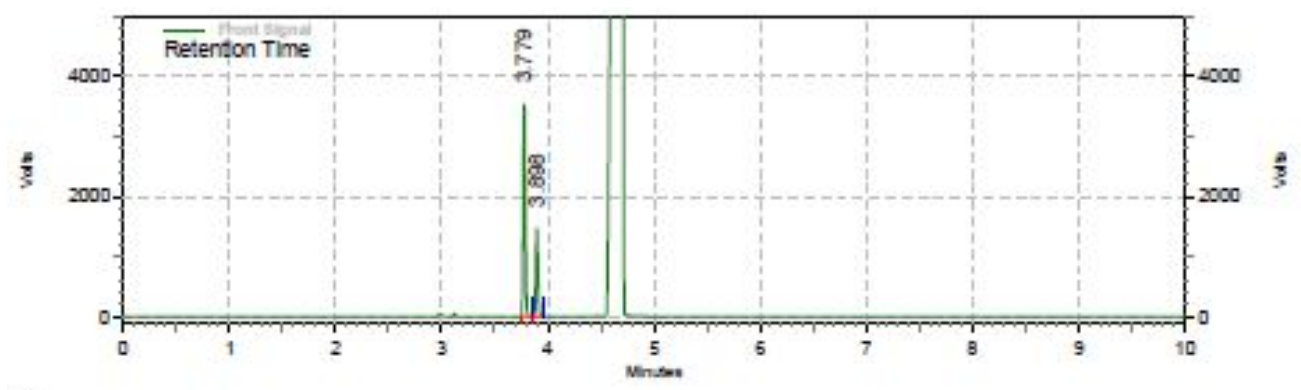

in

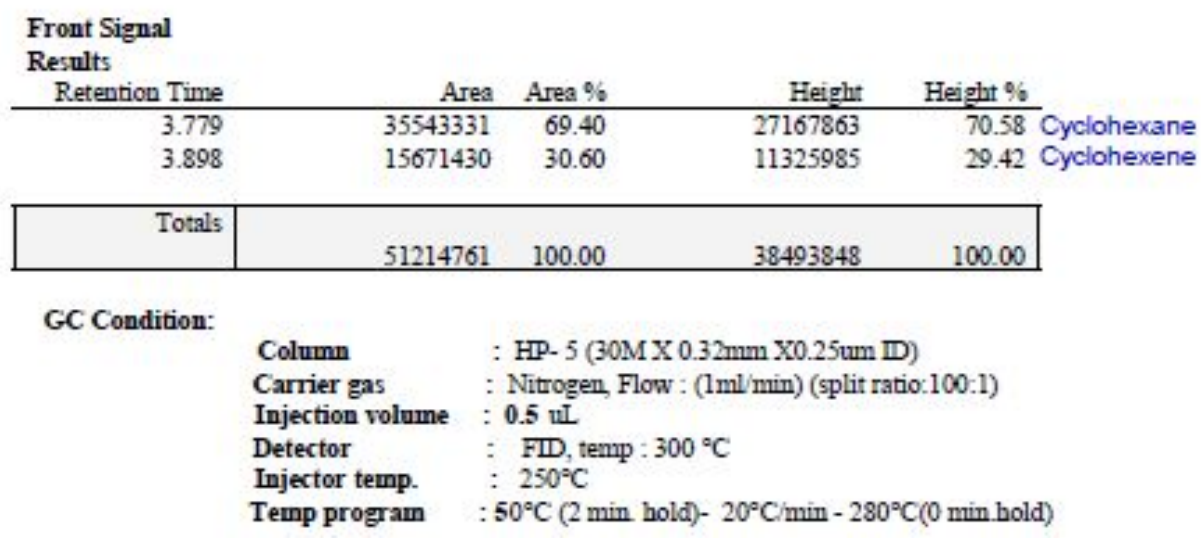

Figure S3B. GC traces for the reaction mixture (without internal standard). 

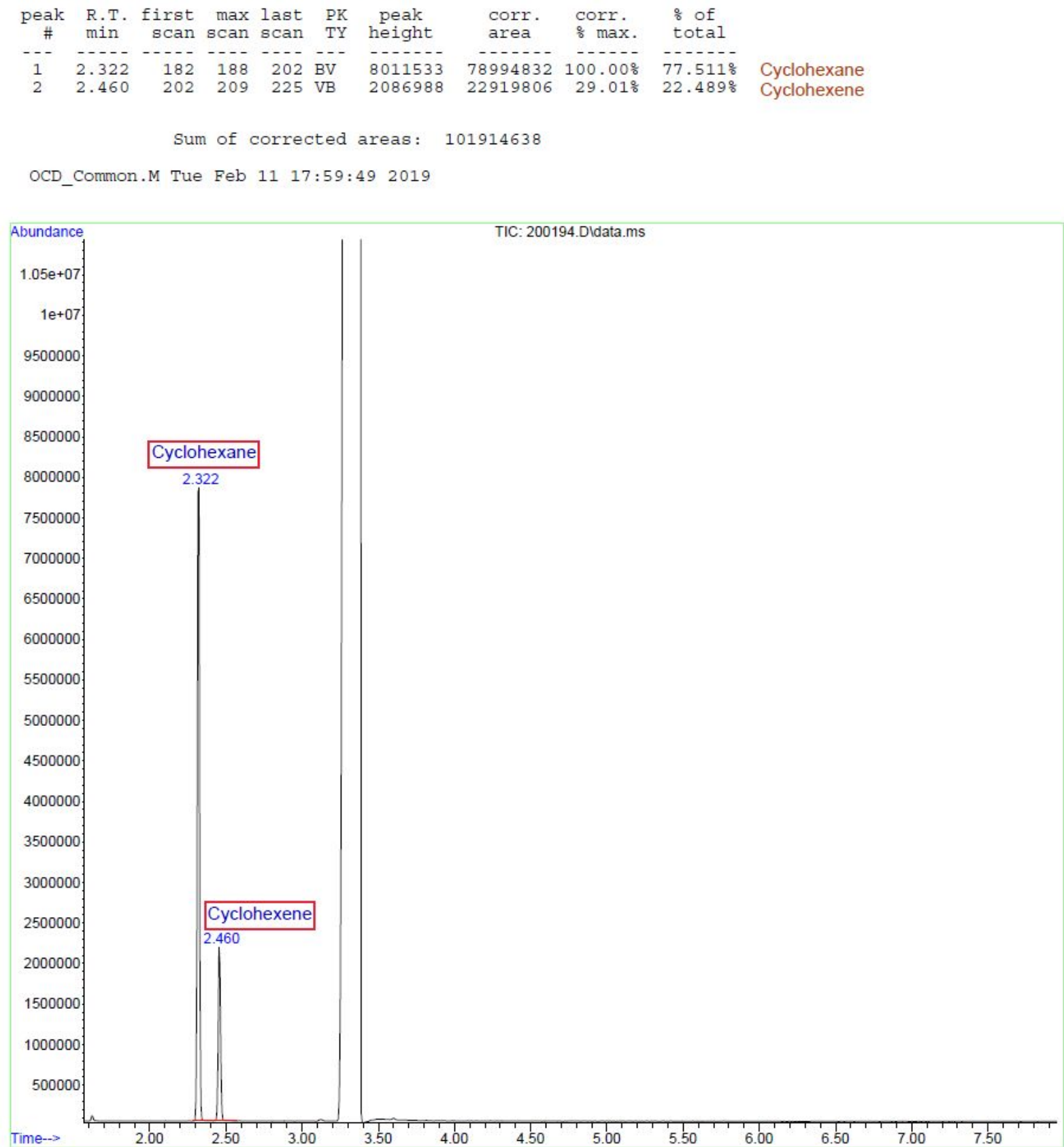

Figure S4A. GC-MS traces for the reaction mixture. 


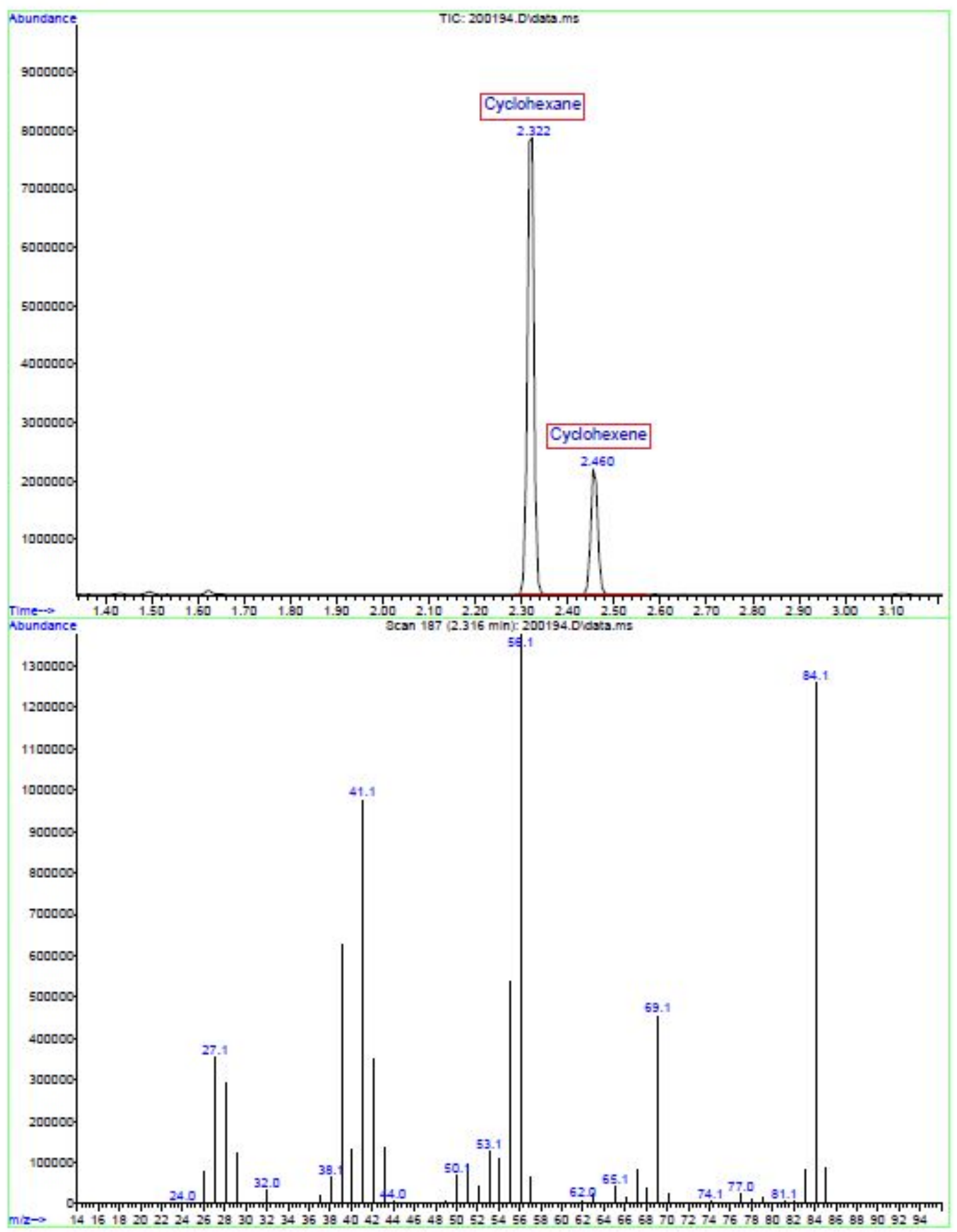

Figure S4B. GC-MS traces for the reaction mixture. 


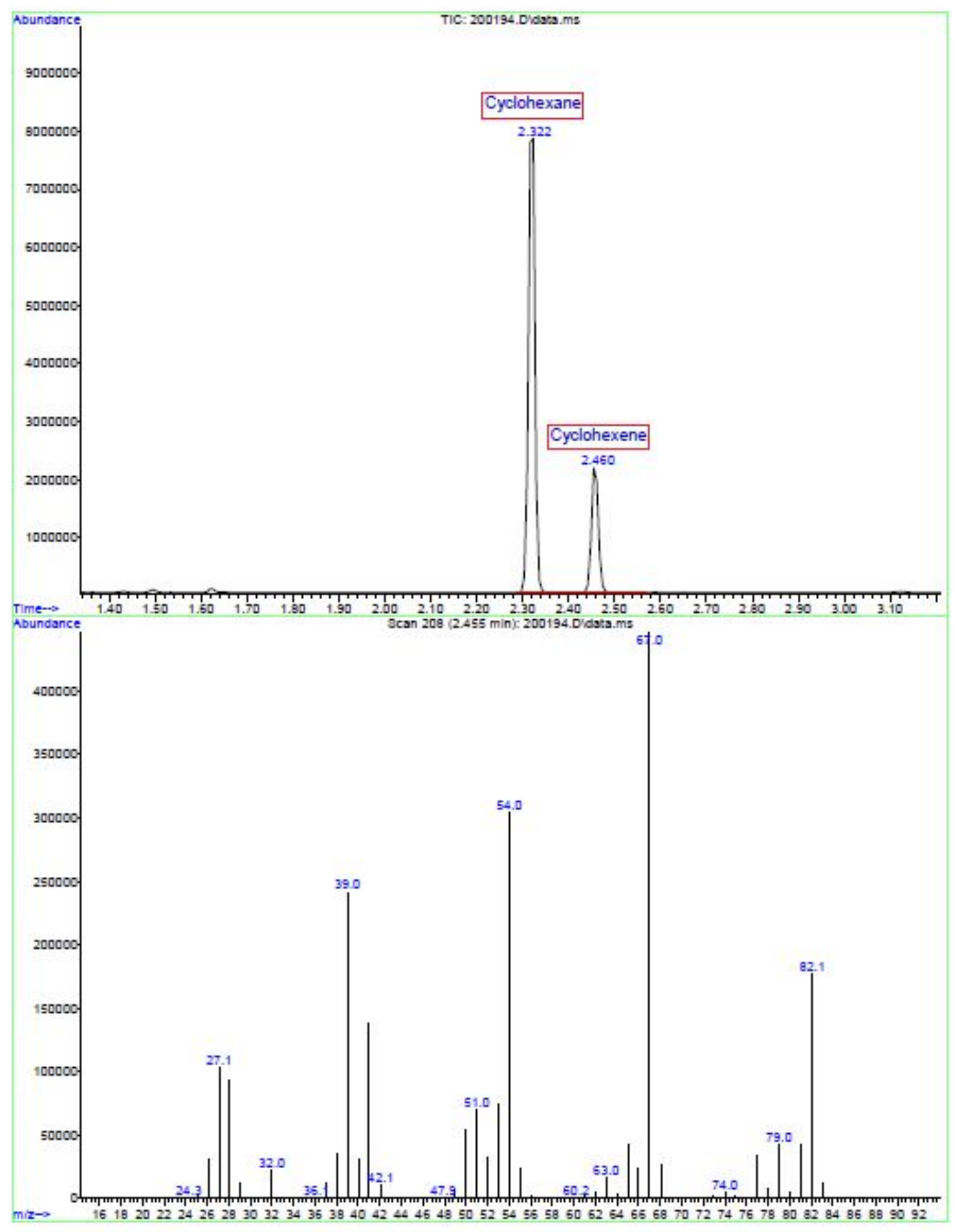

Figure S4C. GC-MS traces for the reaction mixture. 


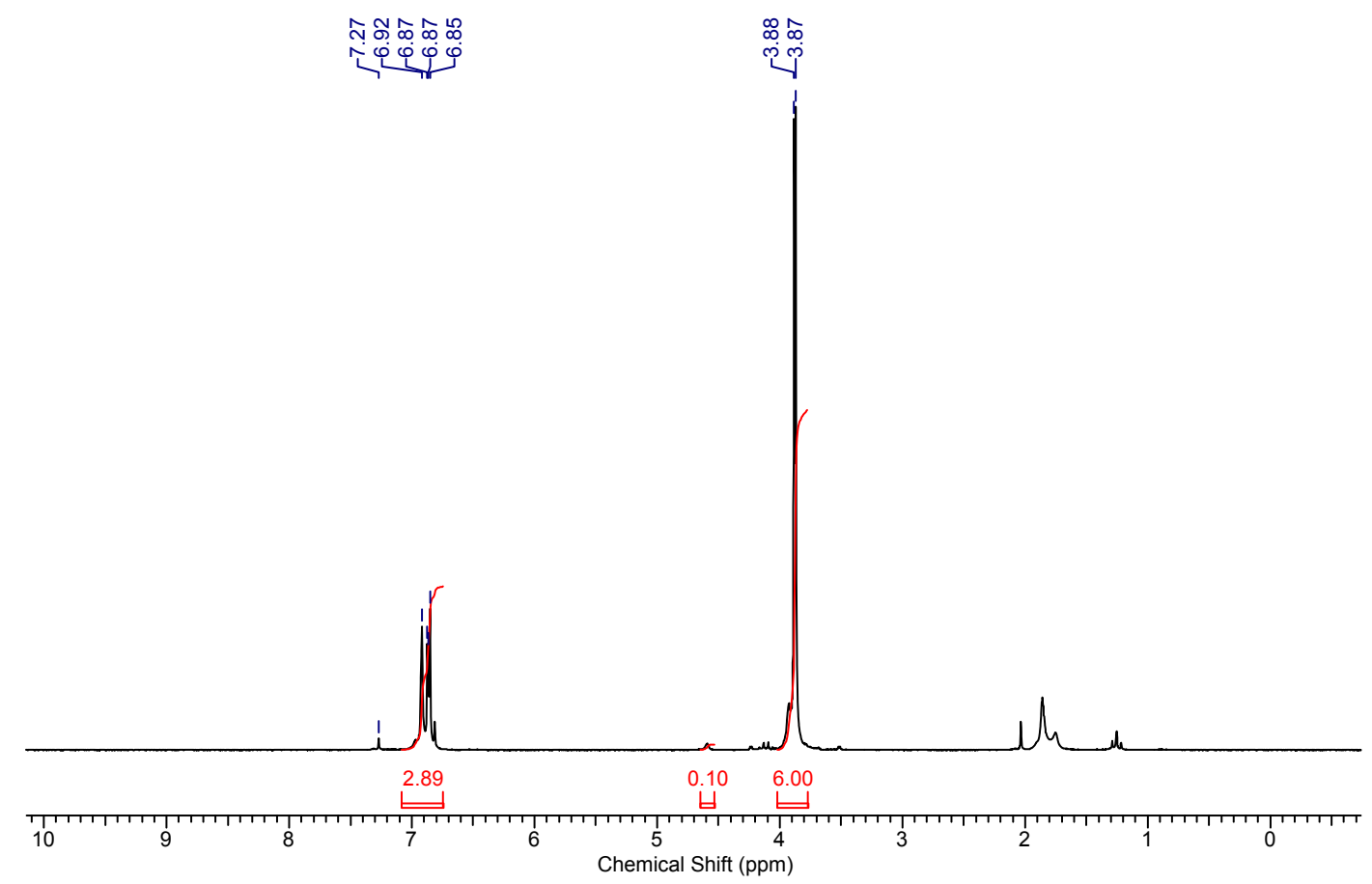

Figure S5. ${ }^{1} \mathrm{H}$ NMR of $1 r-[D]\left(400 \mathrm{MHz}, \mathrm{CDCl}_{3}\right)$

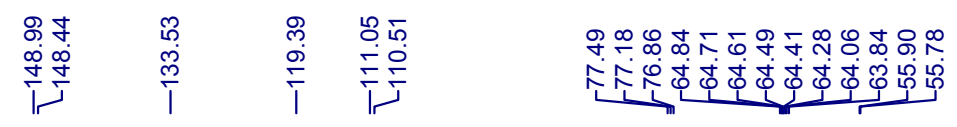

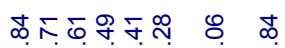

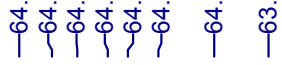

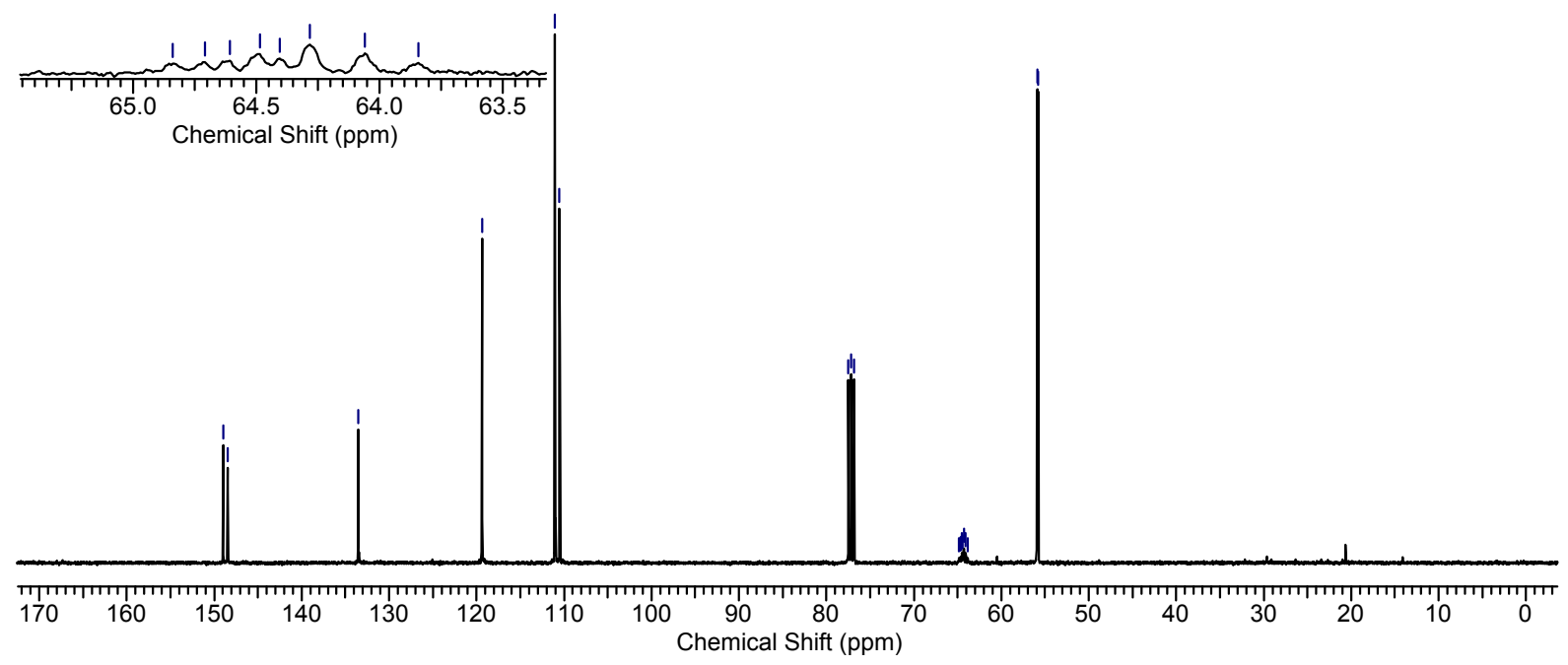

Figure S6. ${ }^{13} \mathrm{C}\left\{{ }^{1} \mathrm{H}\right\} \mathrm{NMR}$ of $\mathbf{1 r}-[\mathbf{D}]\left(101 \mathrm{MHz}, \mathrm{CDCl}_{3}\right)$ 


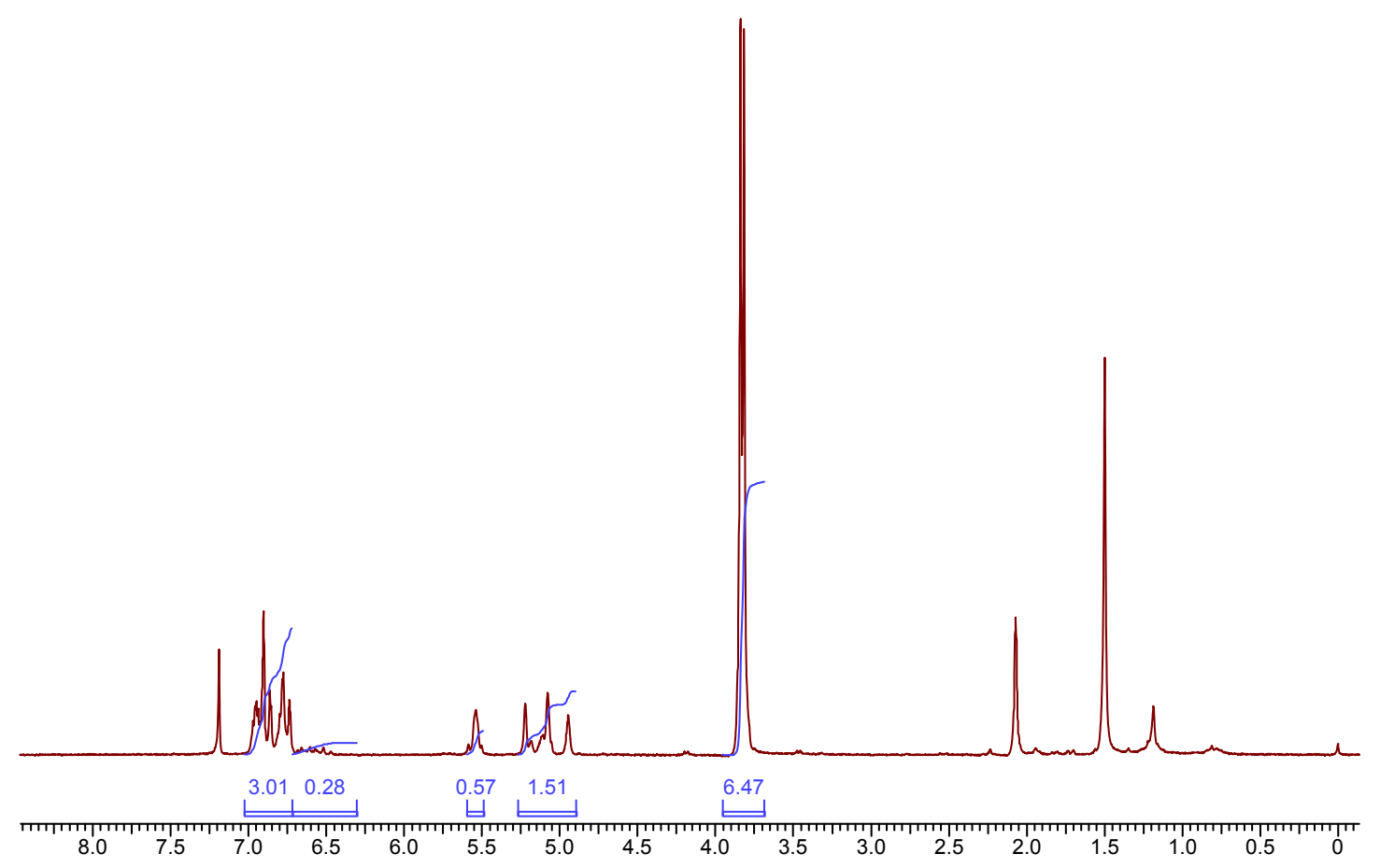

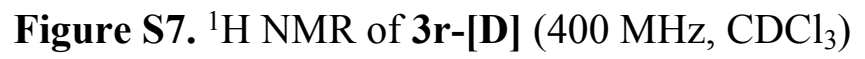
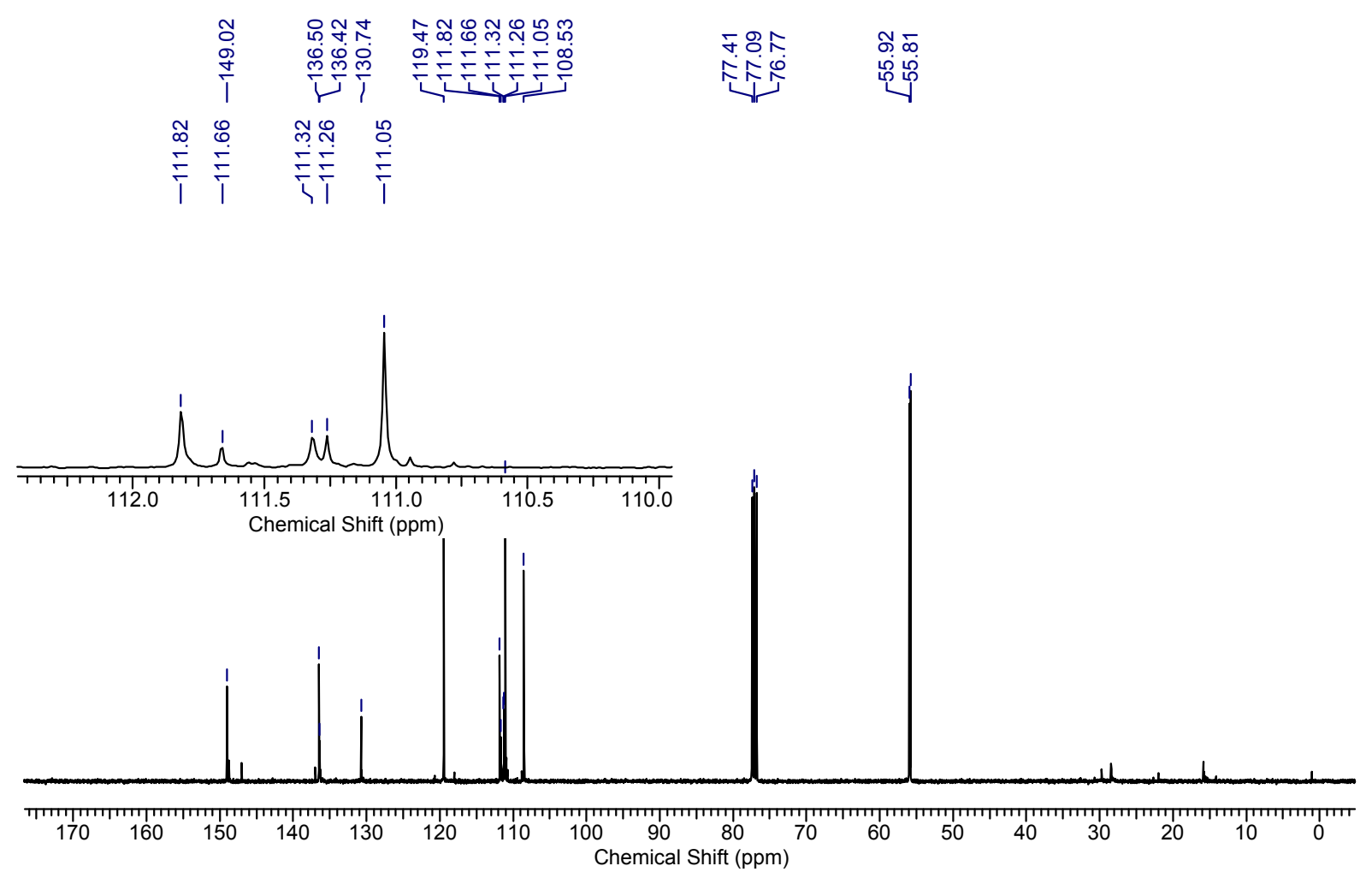

Figure S8. ${ }^{13} \mathrm{C}\left\{{ }^{1} \mathrm{H}\right\} \mathrm{NMR}$ of $\mathbf{3 r}-[\mathbf{D}]\left(101 \mathrm{MHz}, \mathrm{CDCl}_{3}\right)$ 


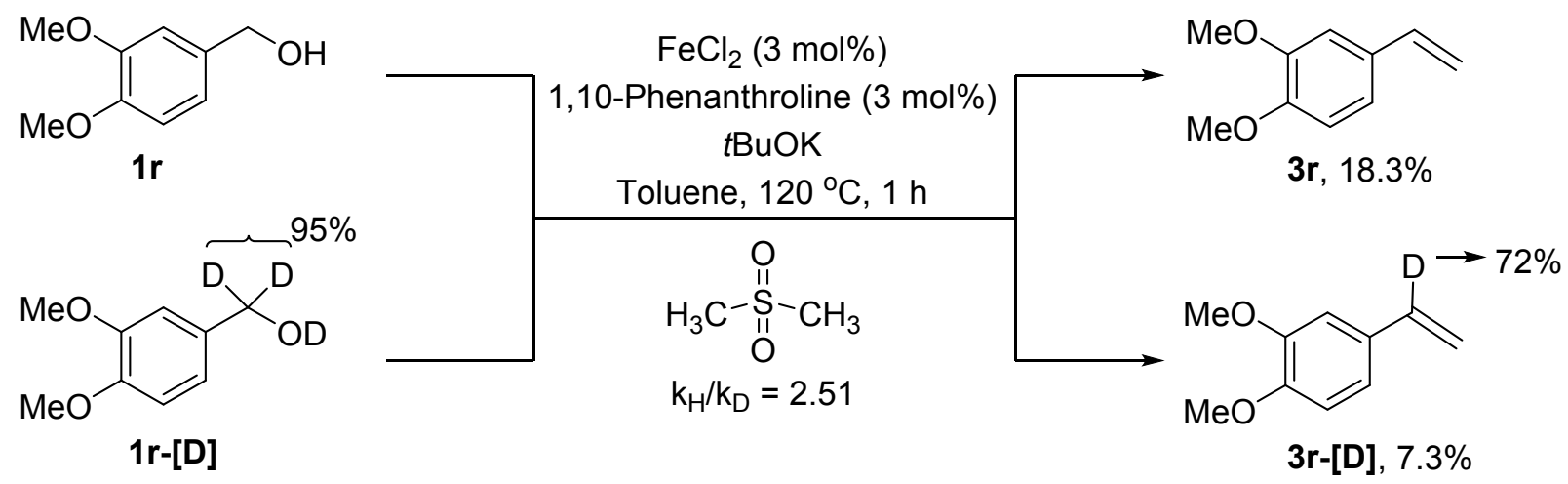

Scheme S1. Competitive KIE experiment: Parallel reaction 


\section{COPY OF NMR}

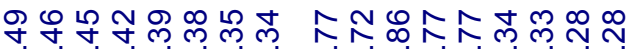

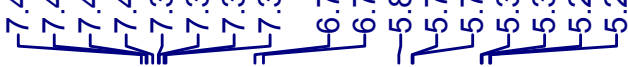

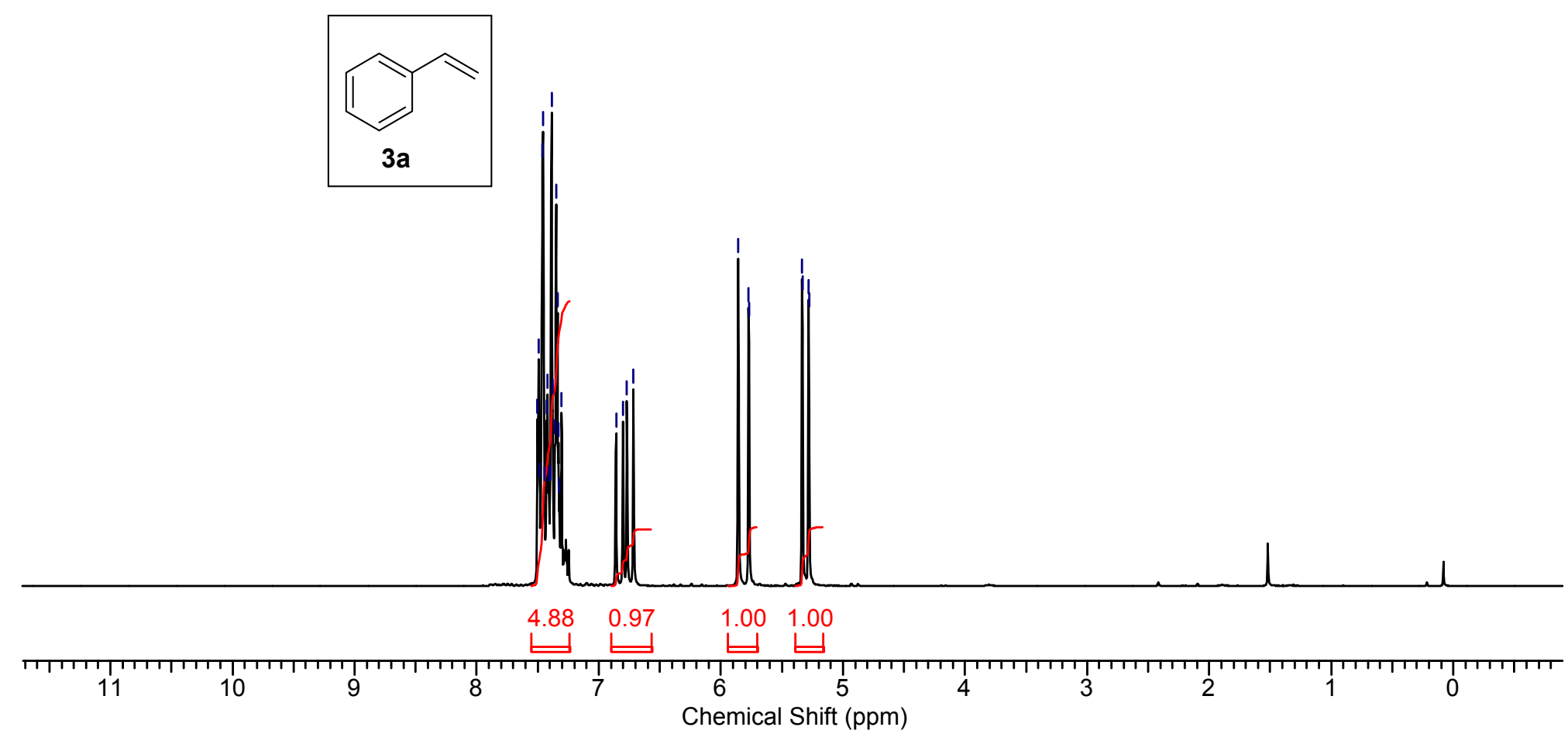

${ }^{1} \mathrm{H}$ NMR of 3a $\left(200 \mathrm{MHz}, \mathrm{CDCl}_{3}\right)$ 


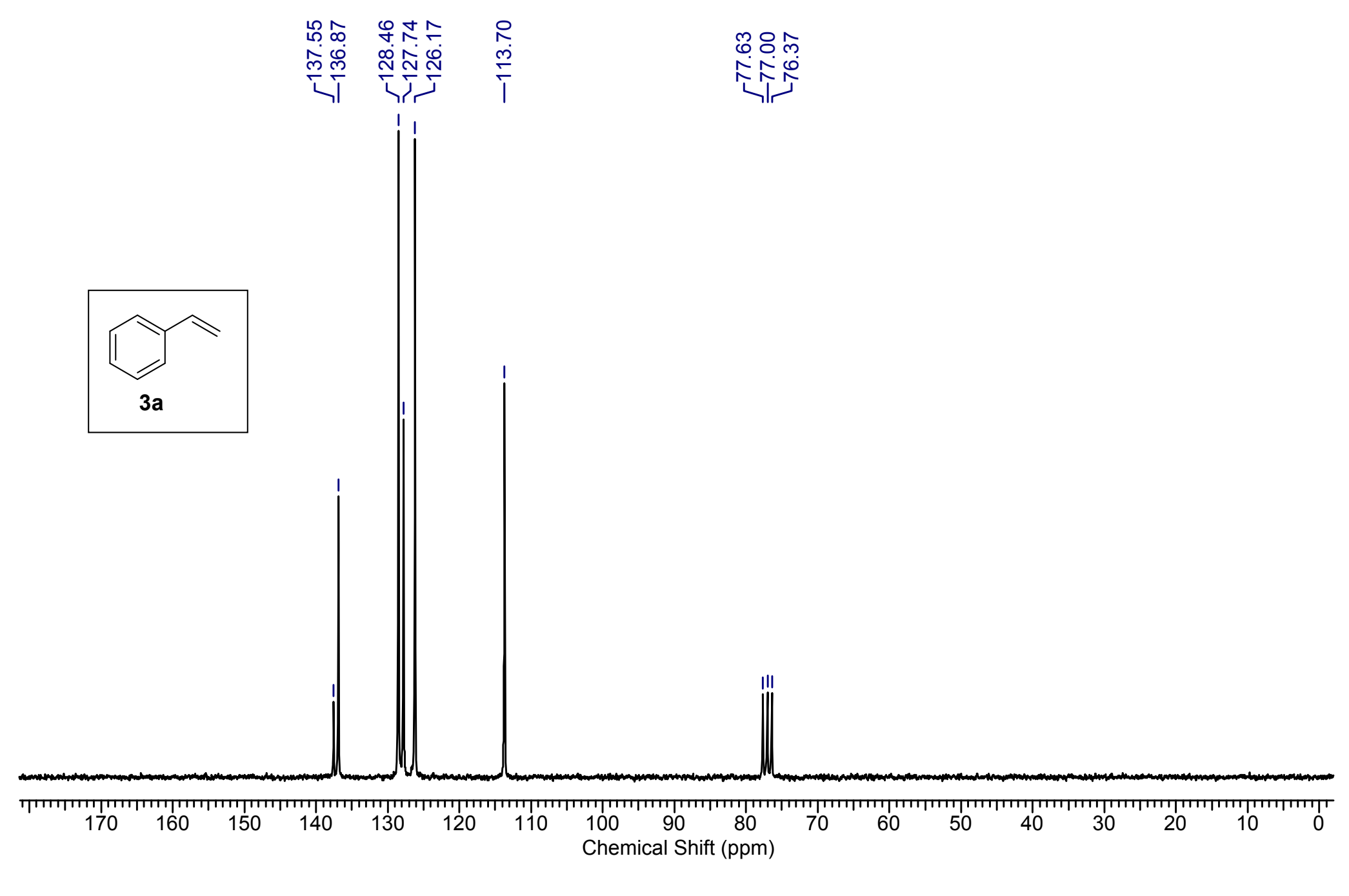

${ }^{13} \mathrm{C}\left\{{ }^{1} \mathrm{H}\right\}$ NMR of $\mathbf{3 a}\left(50 \mathrm{MHz}, \mathrm{CDCl}_{3}\right)$ 


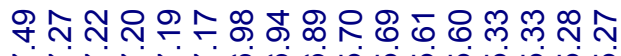

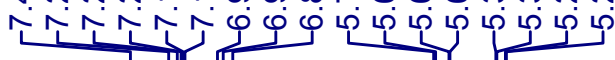

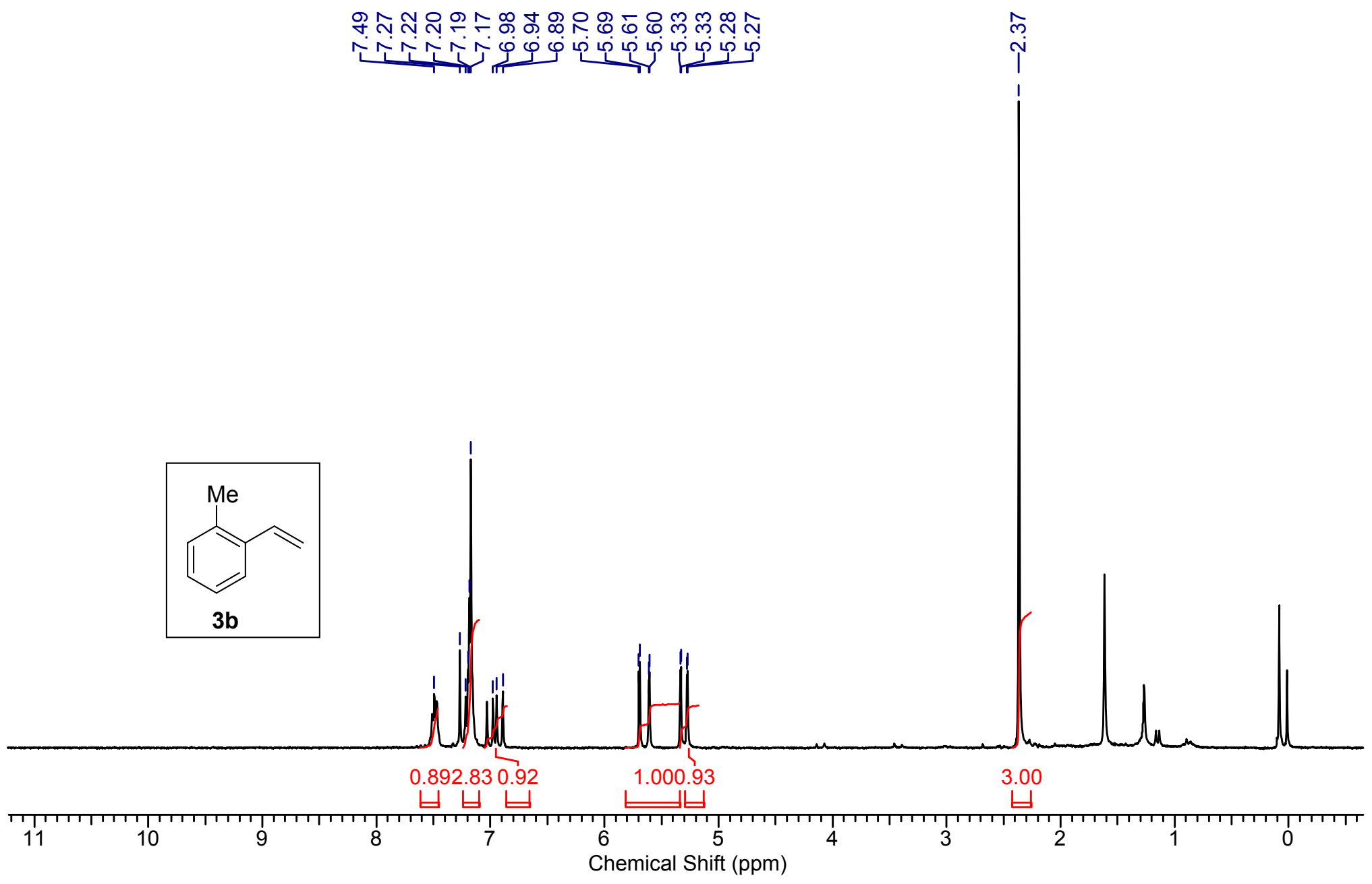

${ }^{1} \mathrm{H}$ NMR of $\mathbf{3 b}\left(200 \mathrm{MHz}, \mathrm{CDCl}_{3}\right)$ 


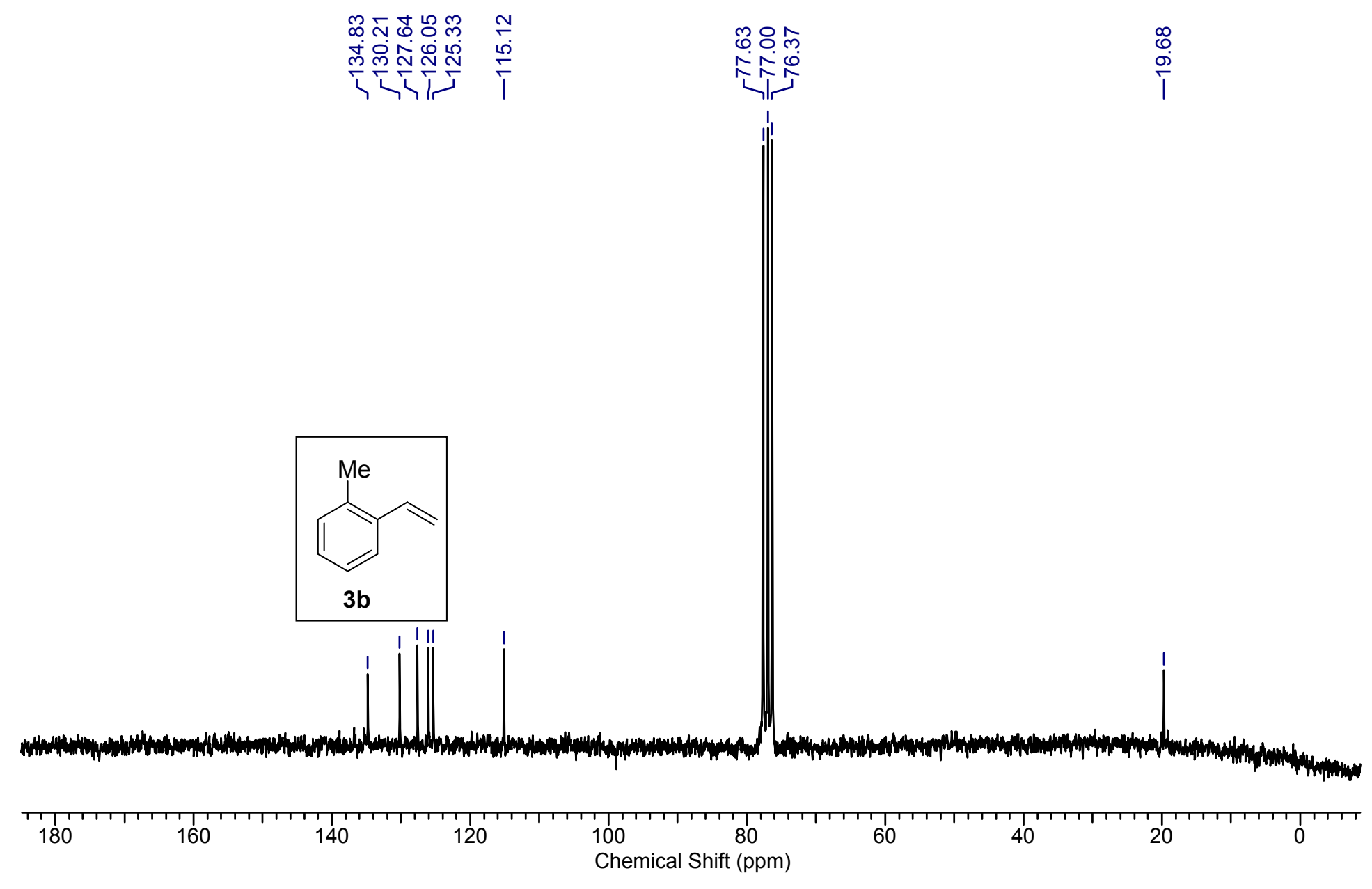

${ }^{13} \mathrm{C}\left\{{ }^{1} \mathrm{H}\right\}$ NMR of $\mathbf{3 b}\left(50 \mathrm{MHz}, \mathrm{CDCl}_{3}\right)$ 


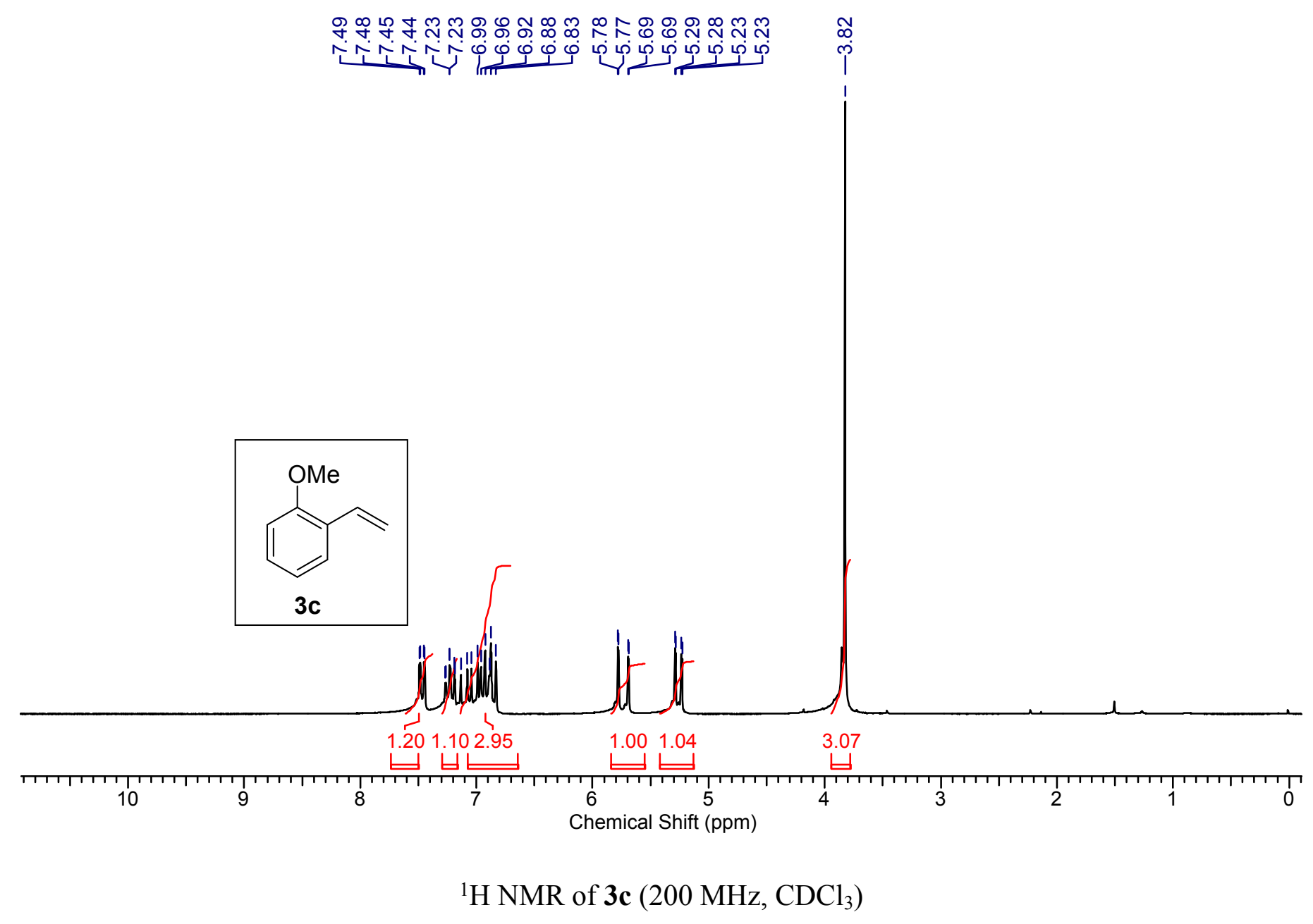




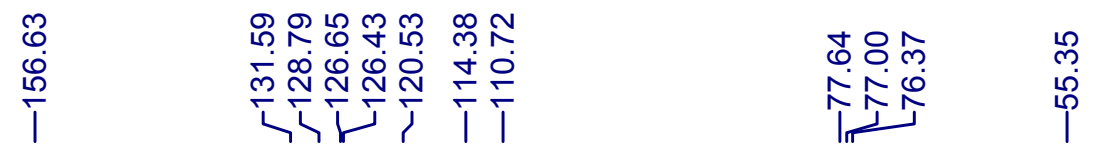
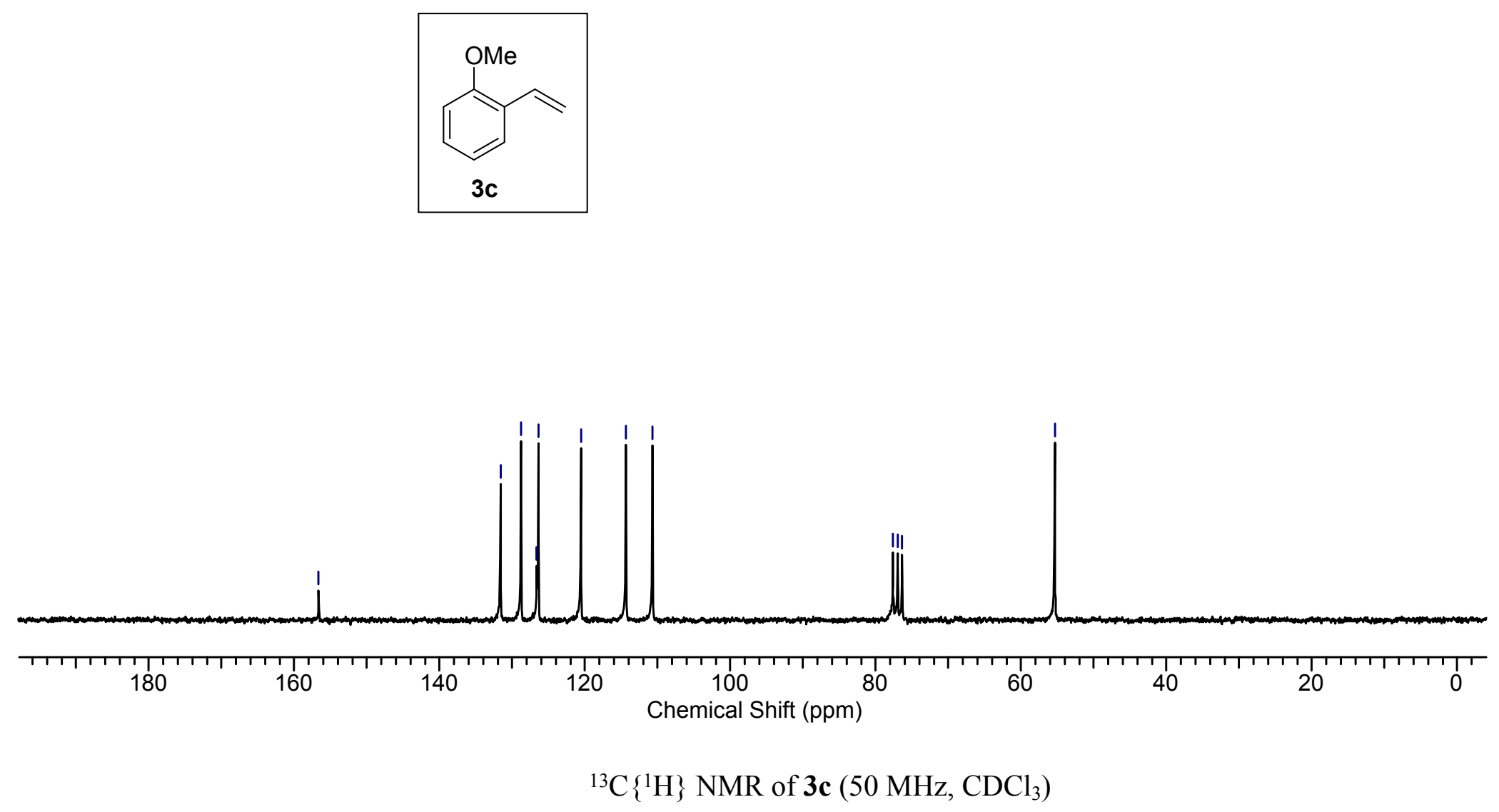


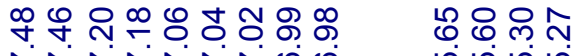

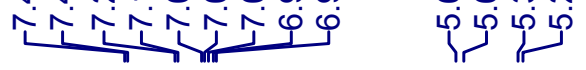
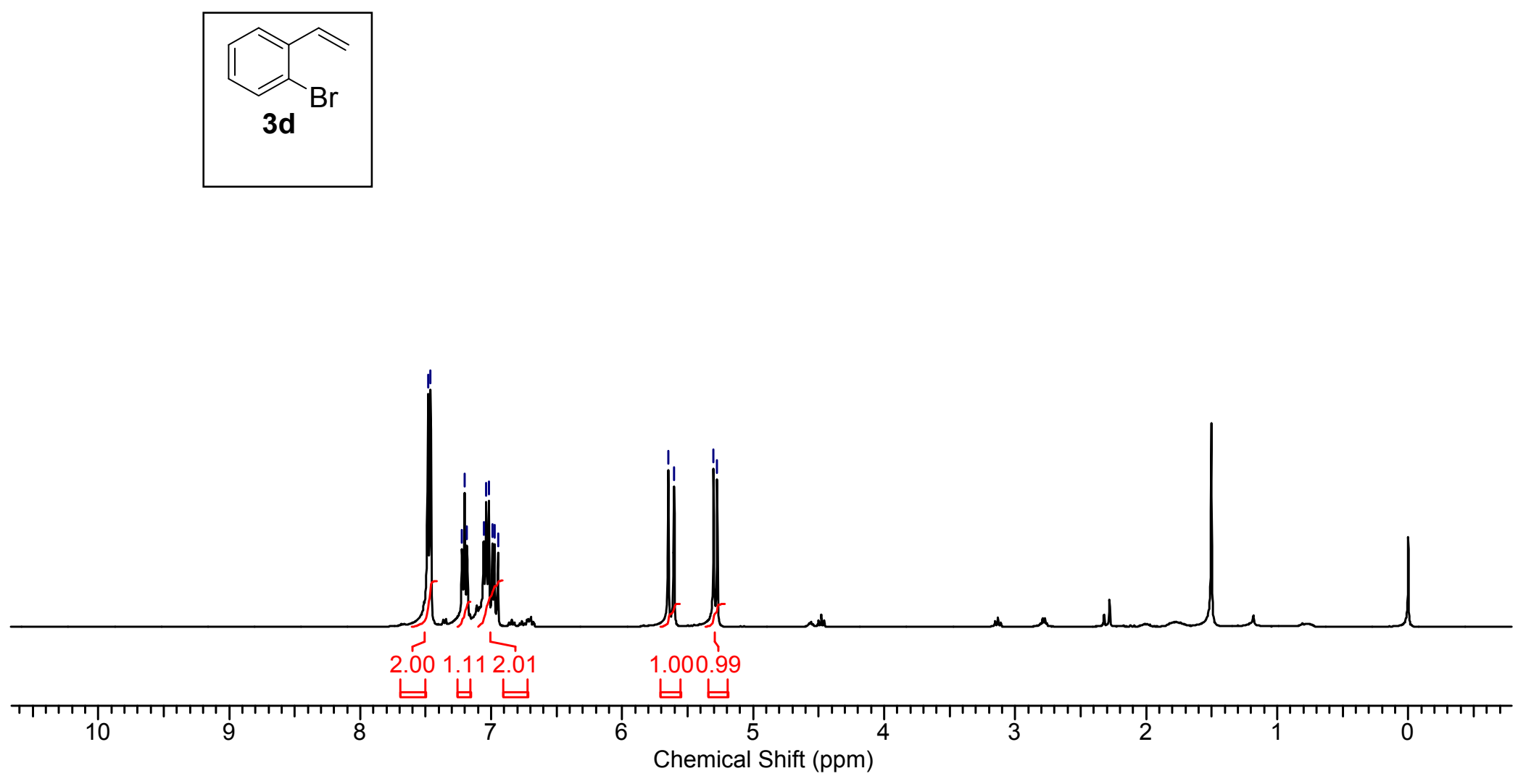

${ }^{1} \mathrm{H}$ NMR of $3 \mathbf{d}\left(400 \mathrm{MHz}, \mathrm{CDCl}_{3}\right)$ 


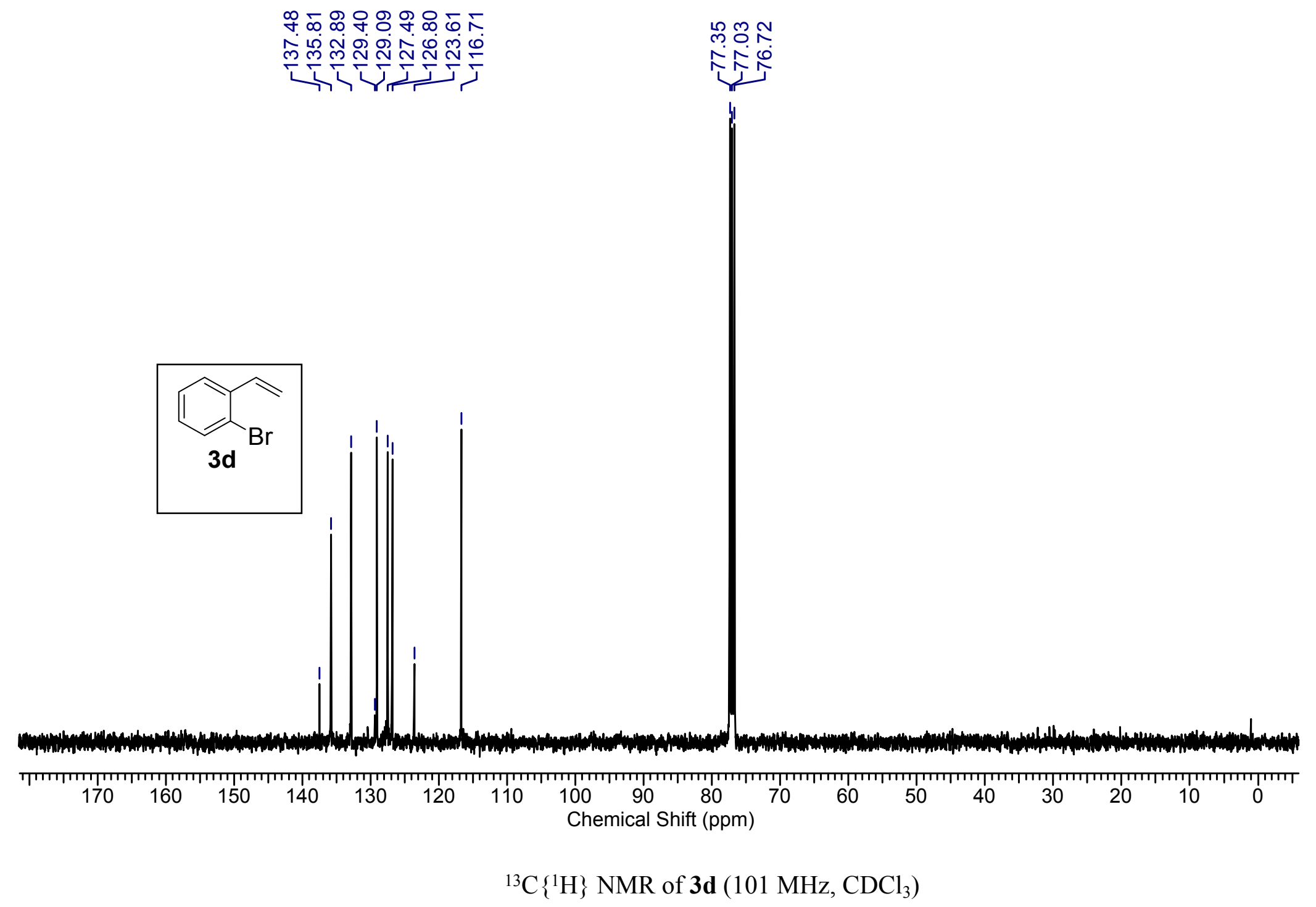




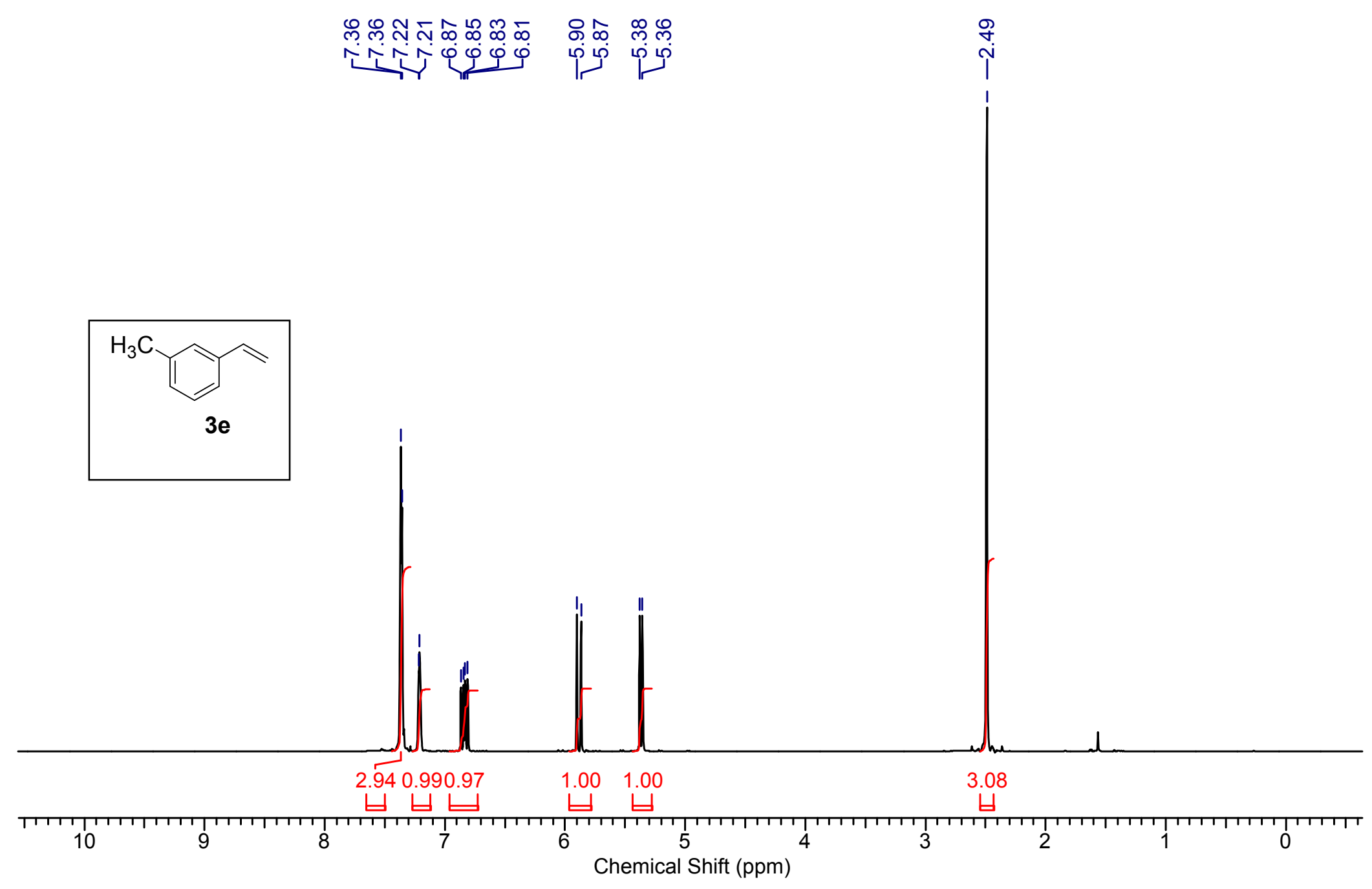

${ }^{1} \mathrm{H}$ NMR of $3 \mathbf{e}\left(500 \mathrm{MHz}, \mathrm{CDCl}_{3}\right)$ 


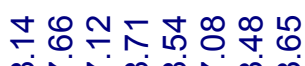
m্

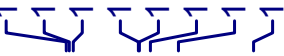

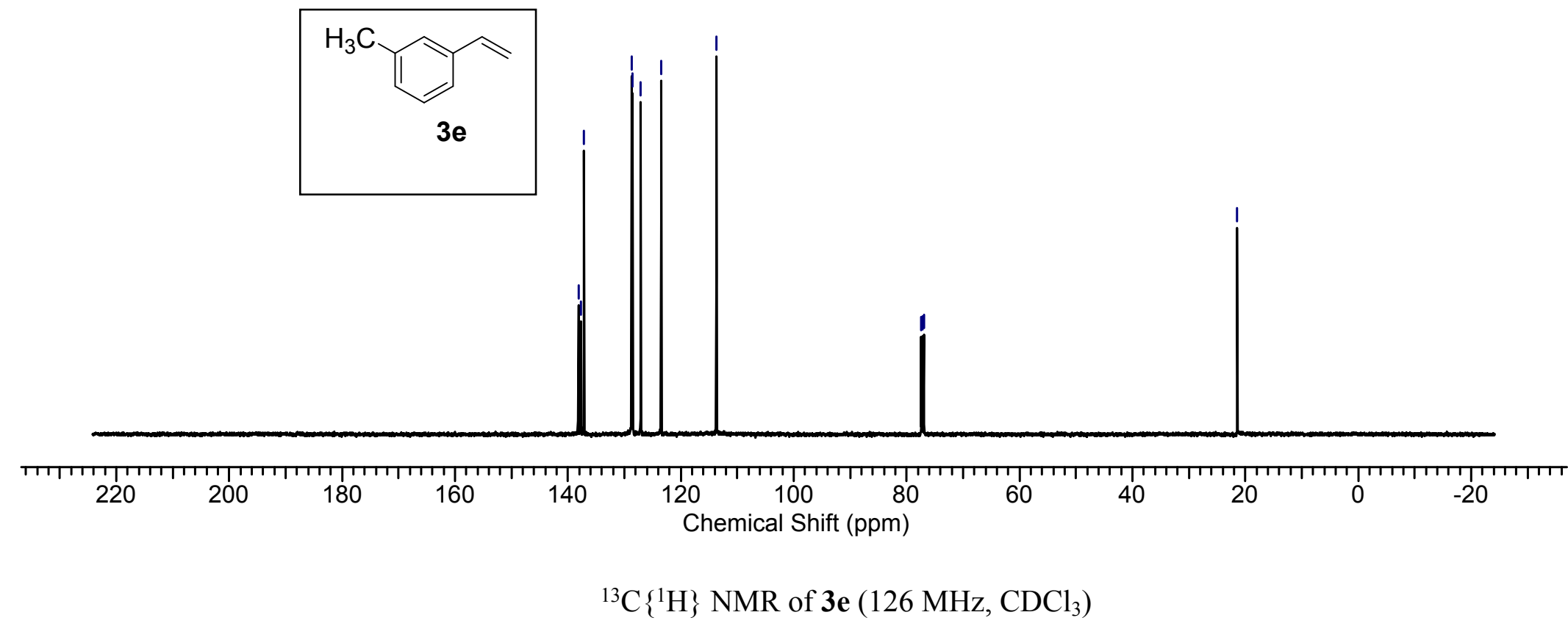




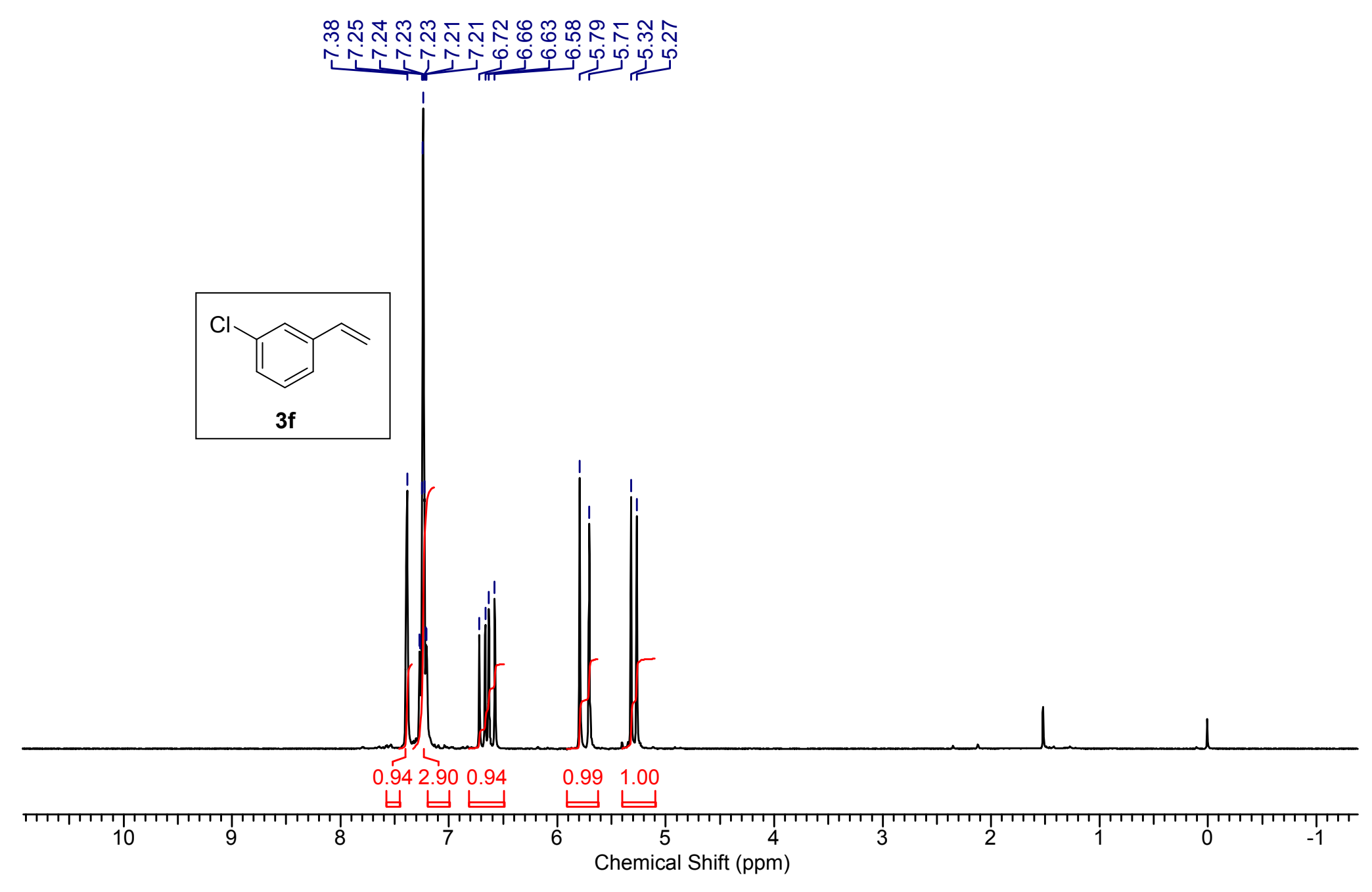

${ }^{1} \mathrm{H}$ NMR of $\mathbf{3 f}\left(200 \mathrm{MHz}, \mathrm{CDCl}_{3}\right)$ 


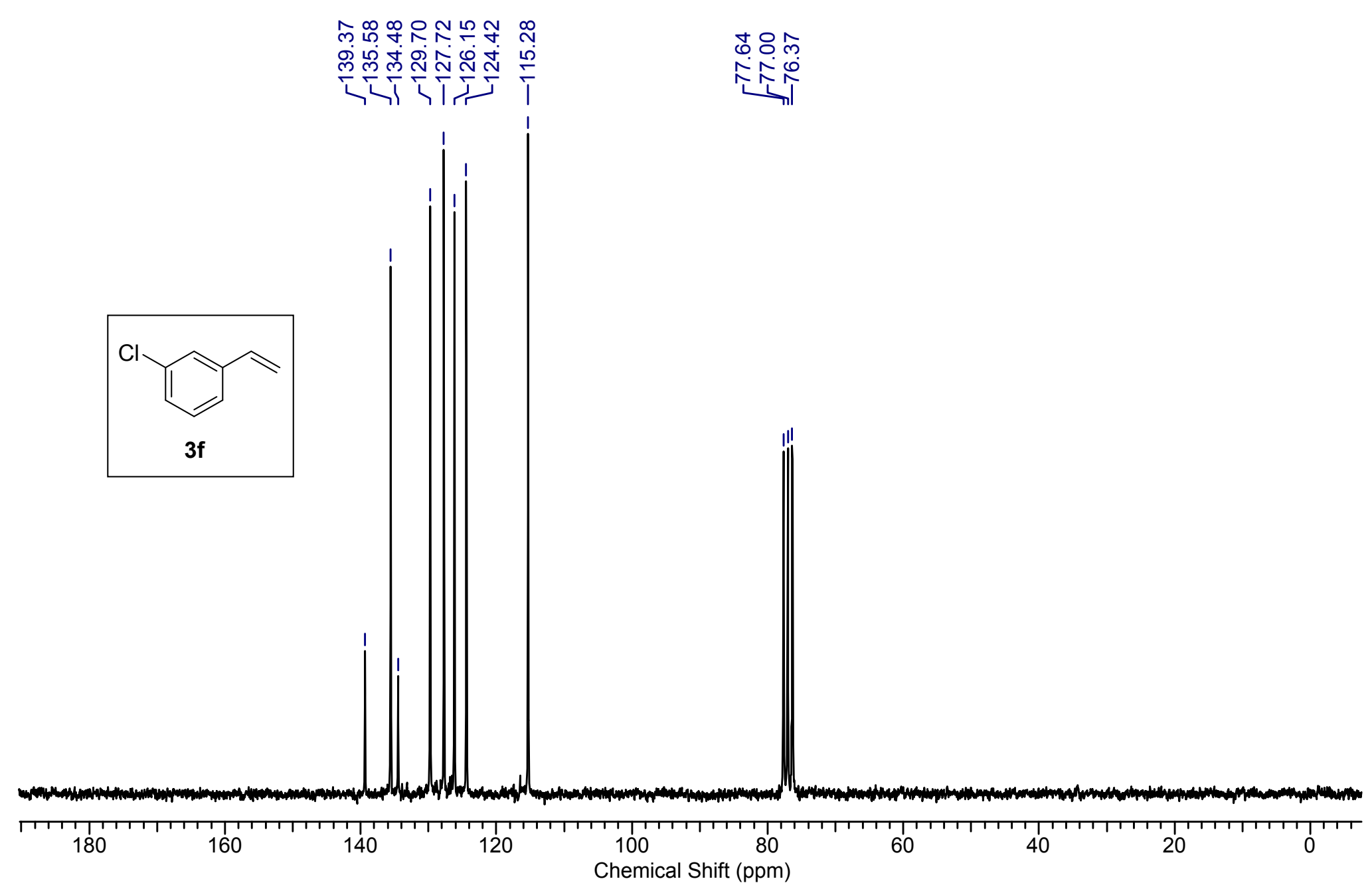

${ }^{13} \mathrm{C}\left\{{ }^{1} \mathrm{H}\right\} \mathrm{NMR}$ of $\mathbf{3 f}\left(50 \mathrm{MHz}, \mathrm{CDCl}_{3}\right)$ 

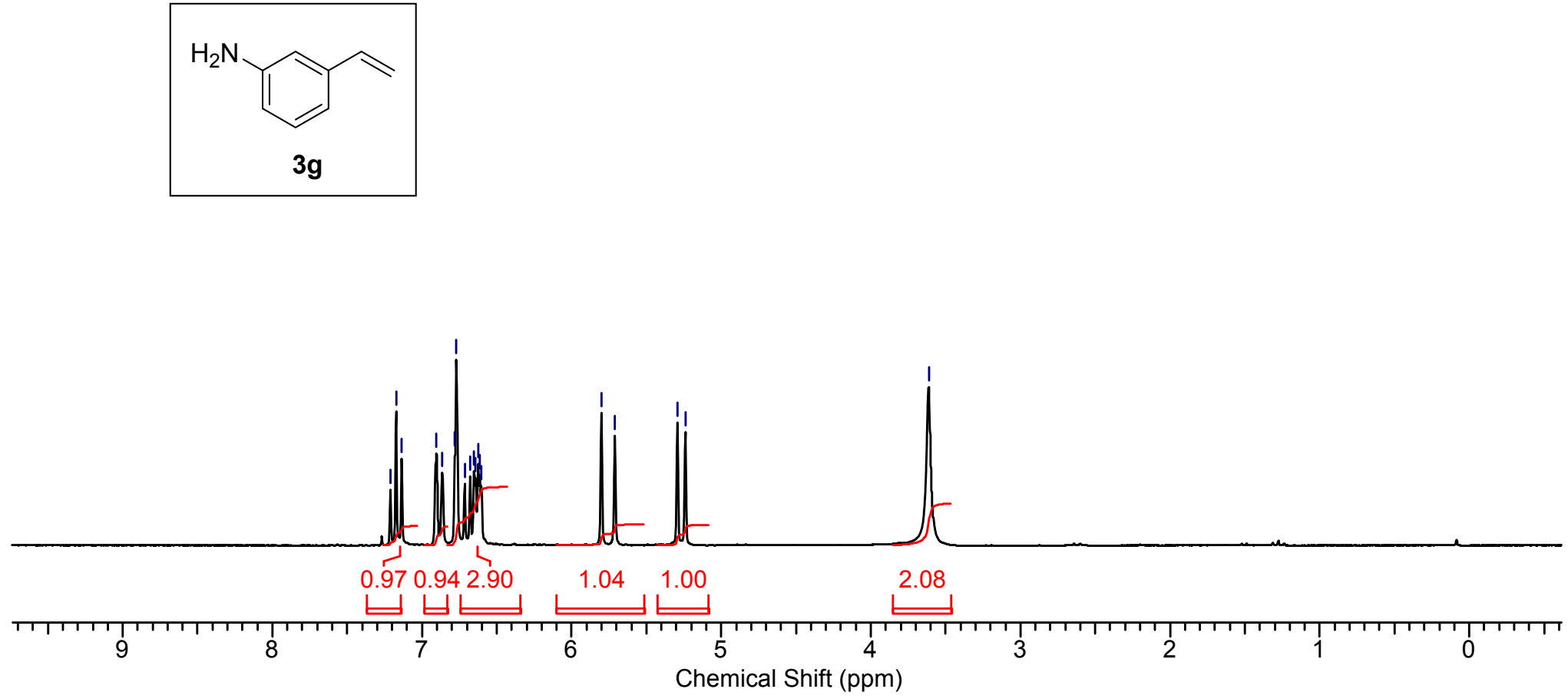

${ }^{1} \mathrm{H}$ NMR of $\mathbf{3 g}\left(200 \mathrm{MHz}, \mathrm{CDCl}_{3}\right)$ 

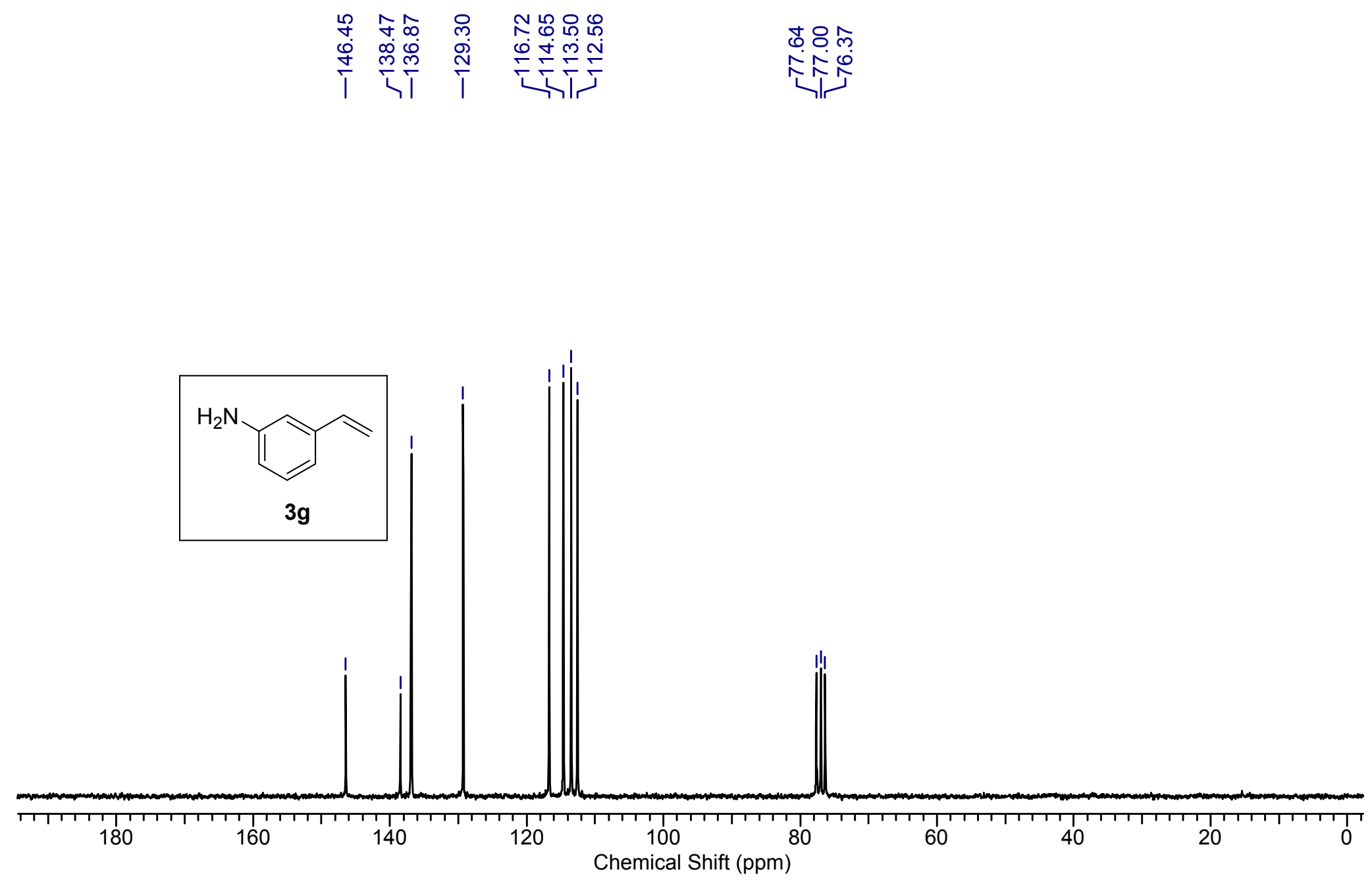

${ }^{13} \mathrm{C}\left\{{ }^{1} \mathrm{H}\right\}$ NMR of $\mathbf{3 g}\left(50 \mathrm{MHz}, \mathrm{CDCl}_{3}\right)$ 


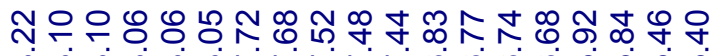

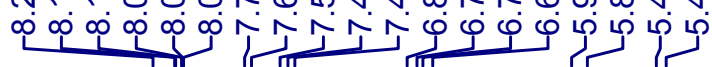
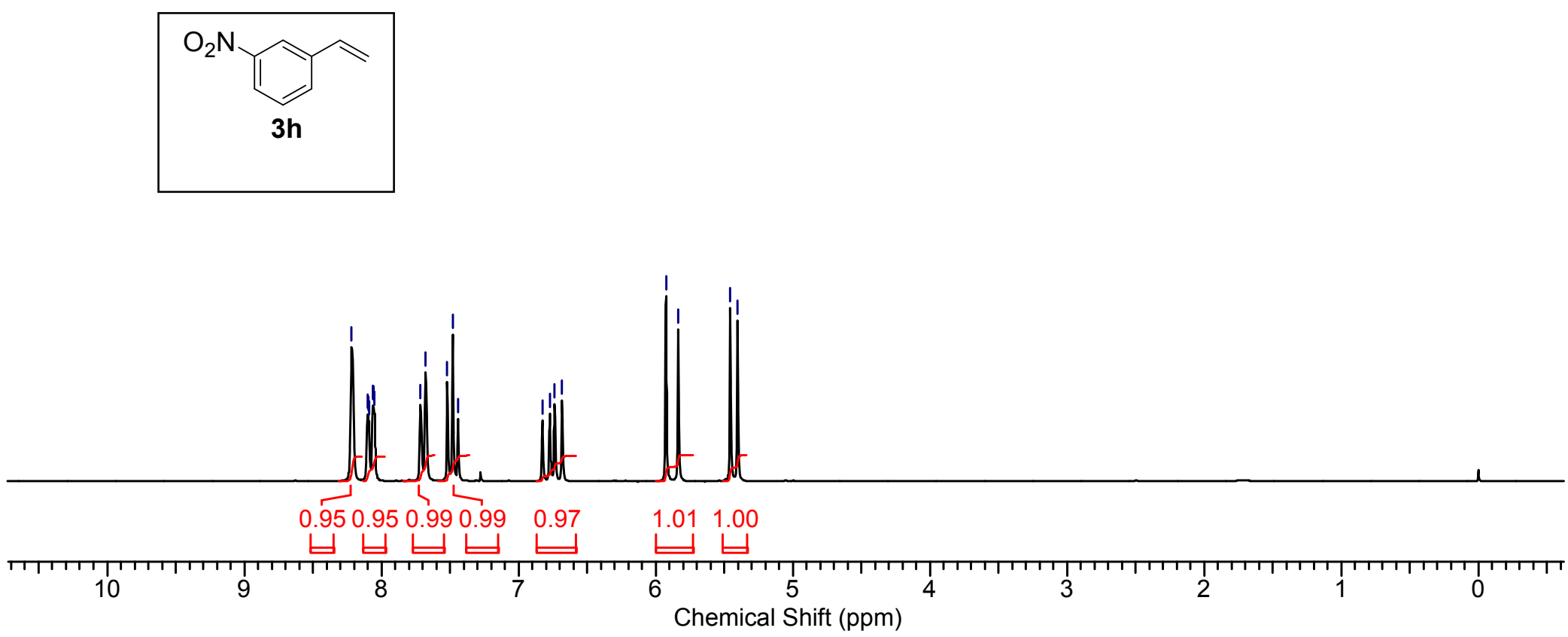

${ }^{1} \mathrm{H}$ NMR of $\mathbf{3 h}\left(200 \mathrm{MHz}, \mathrm{CDCl}_{3}\right)$ 


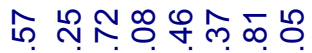

㐫

1

N寸8

손ำ

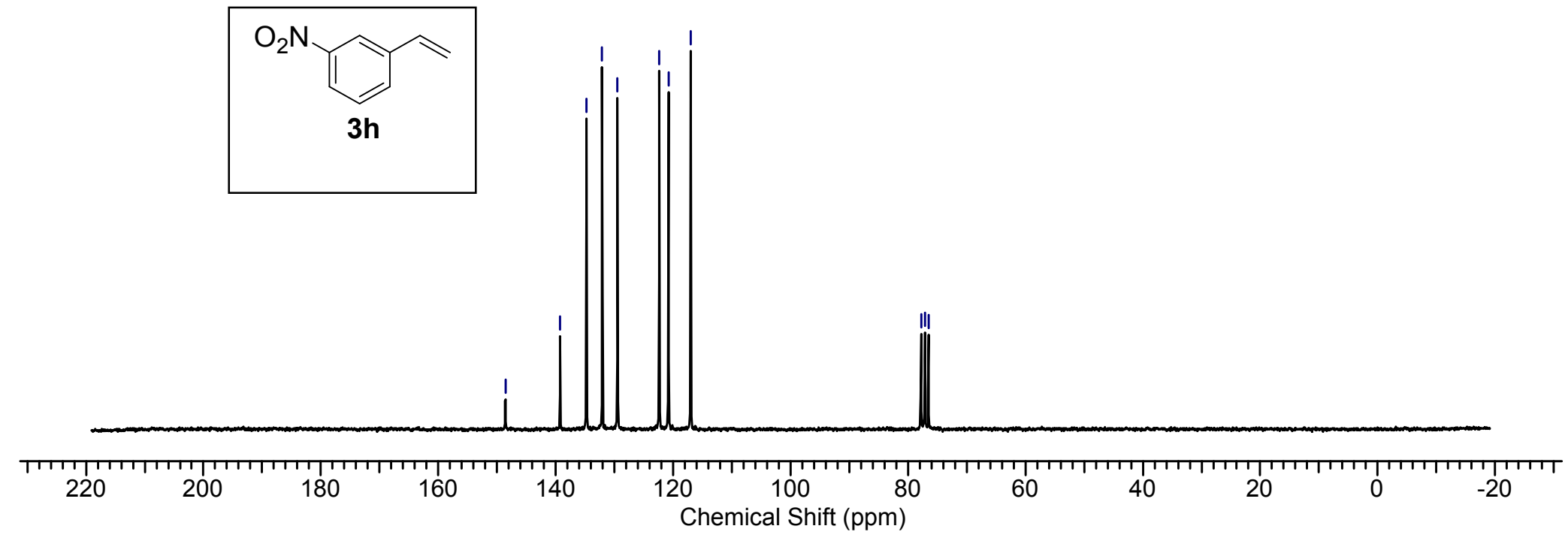

${ }^{13} \mathrm{C}\left\{{ }^{1} \mathrm{H}\right\}$ NMR of $\mathbf{3 h}\left(50 \mathrm{MHz}, \mathrm{CDCl}_{3}\right)$ 


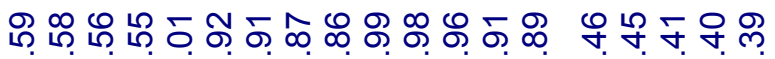

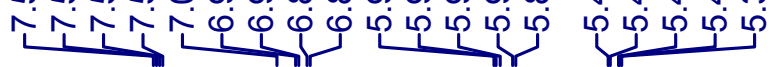

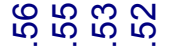
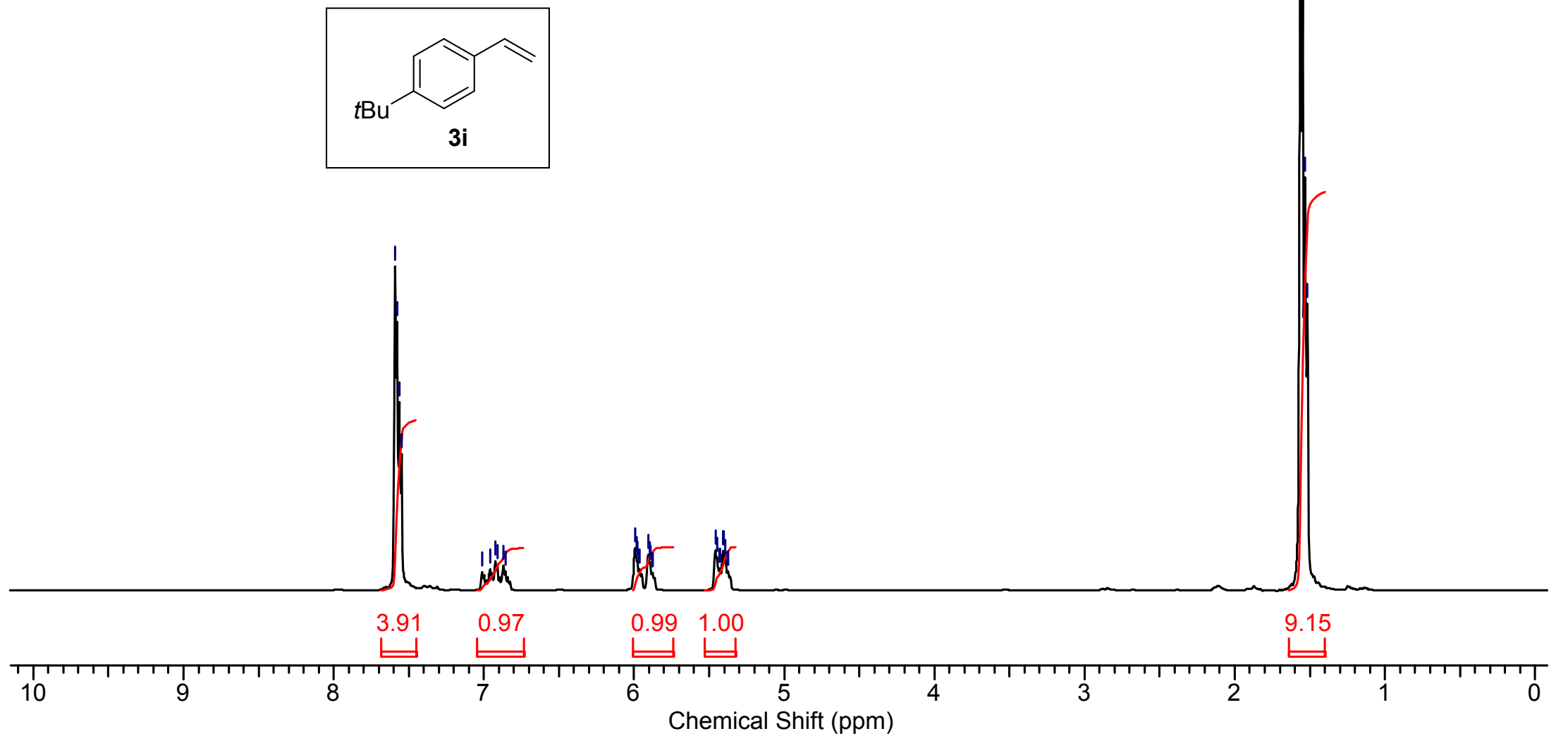

${ }^{1} \mathrm{H}$ NMR of $\mathbf{3 i}\left(200 \mathrm{MHz}, \mathrm{CDCl}_{3}\right)$ 


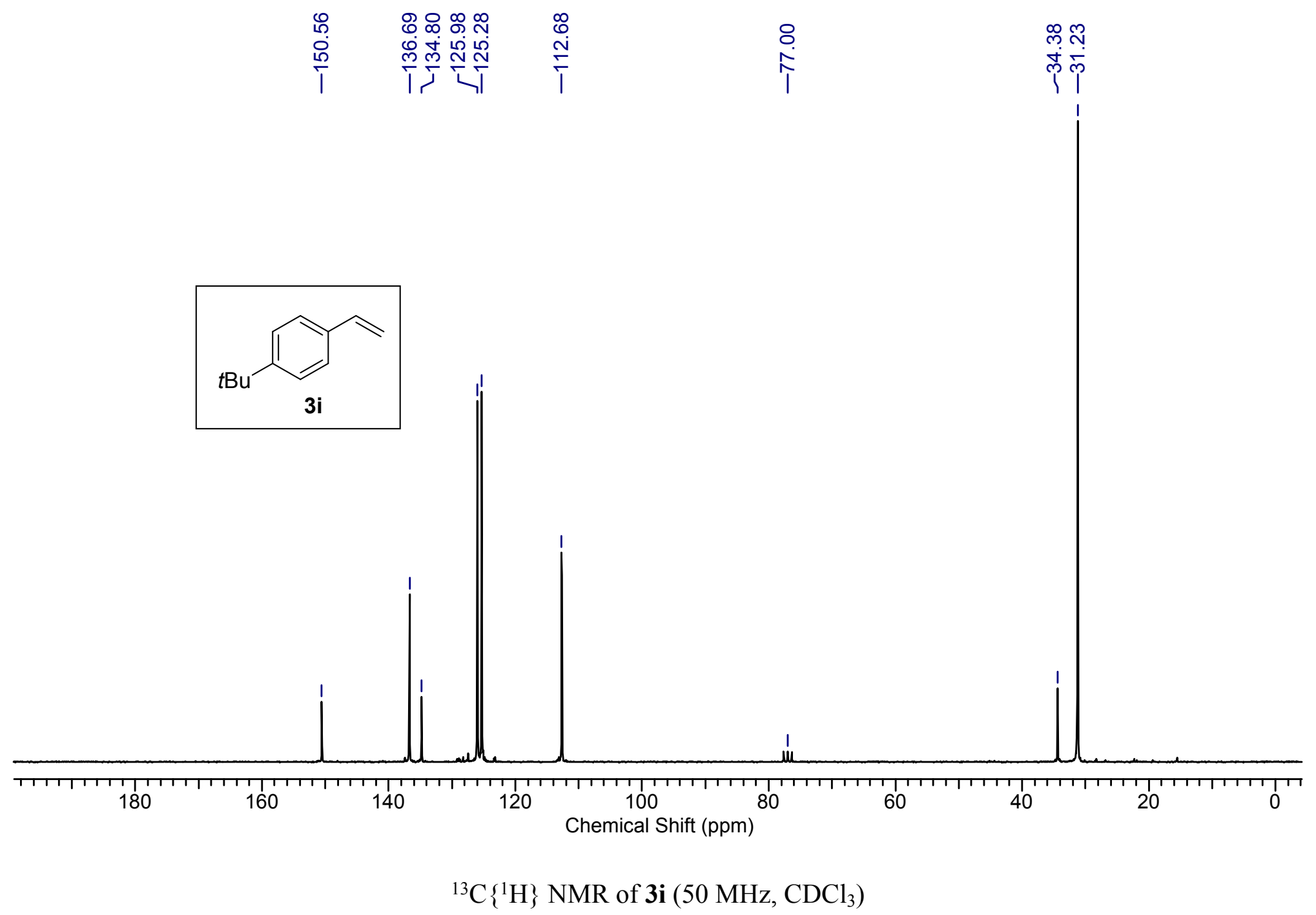




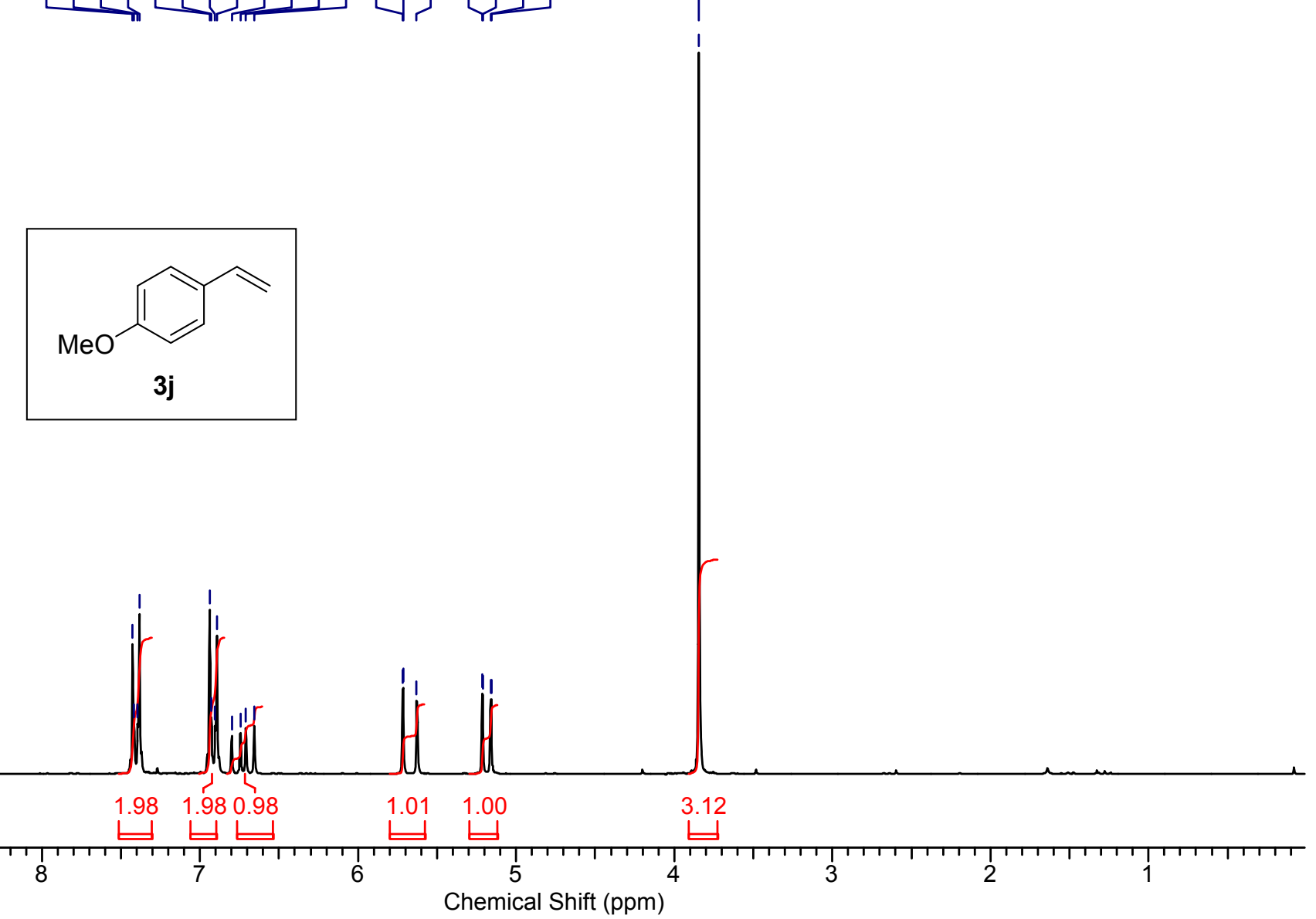

${ }^{1} \mathrm{H}$ NMR of $\mathbf{3 j}\left(200 \mathrm{MHz}, \mathrm{CDCl}_{3}\right)$ 


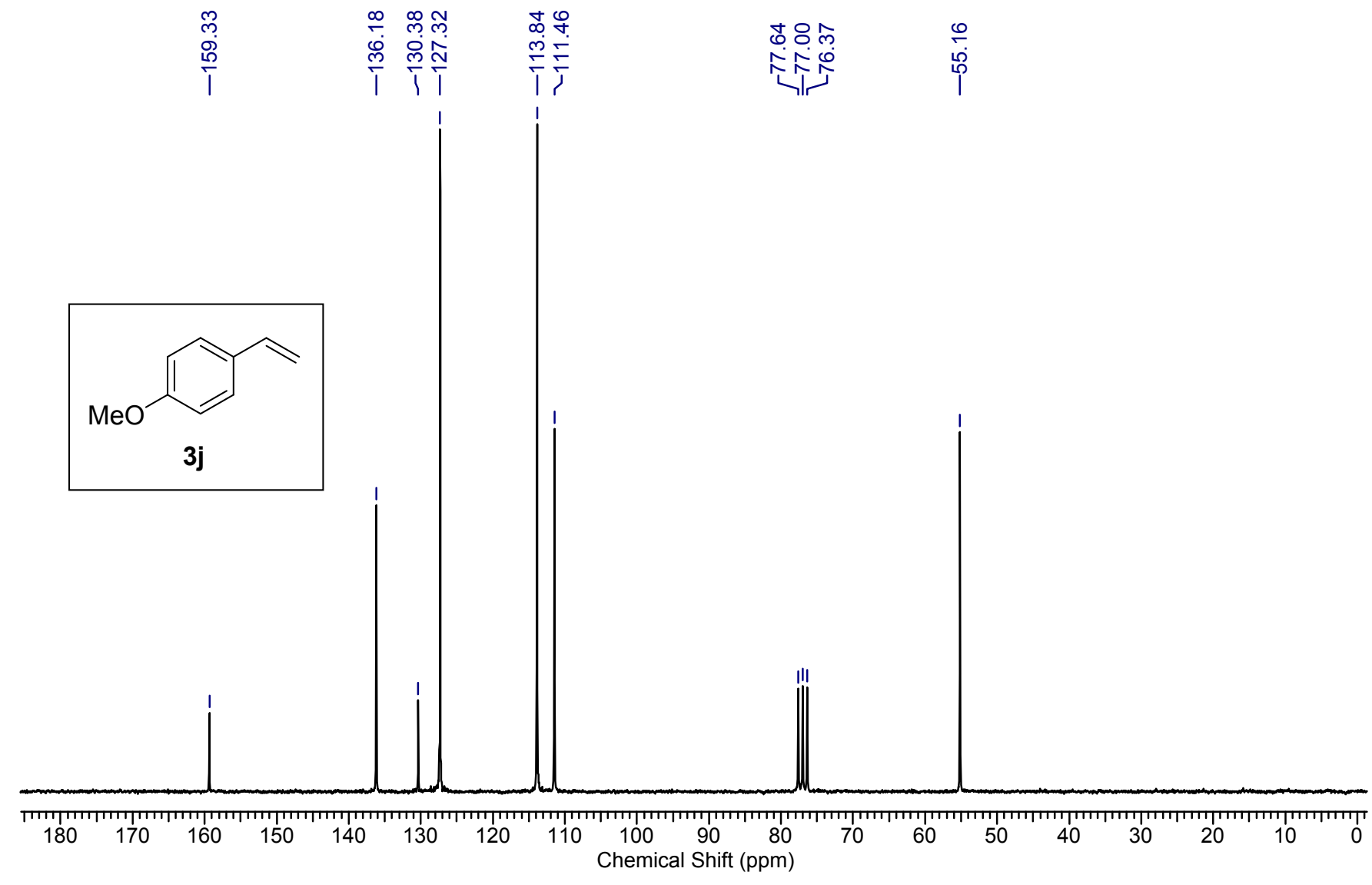

${ }^{13} \mathrm{C}\left\{{ }^{1} \mathrm{H}\right\} \mathrm{NMR}$ of $\mathbf{3 j}\left(50 \mathrm{MHz}, \mathrm{CDCl}_{3}\right)$ 

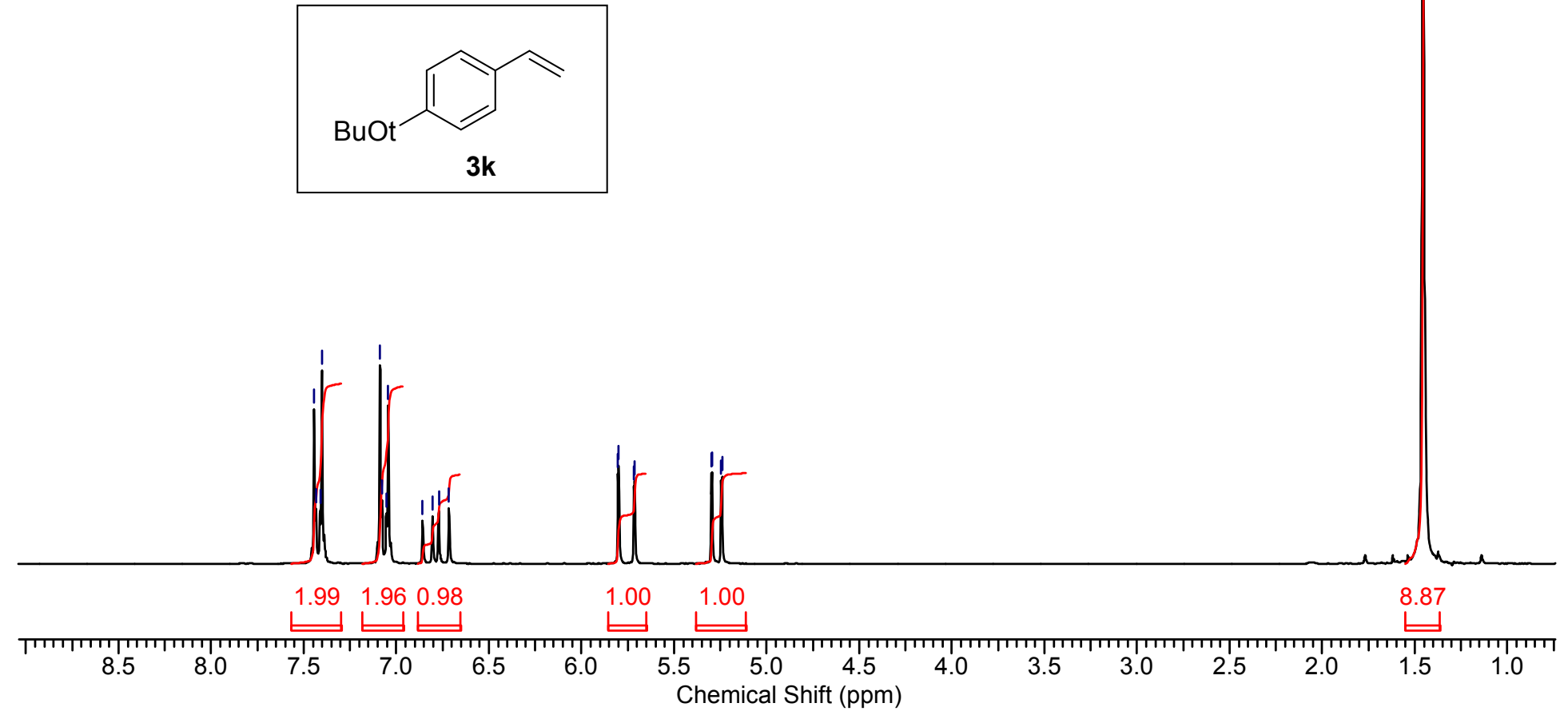

${ }^{1} \mathrm{H}$ NMR of 3k $\left(200 \mathrm{MHz}, \mathrm{CDCl}_{3}\right)$ 


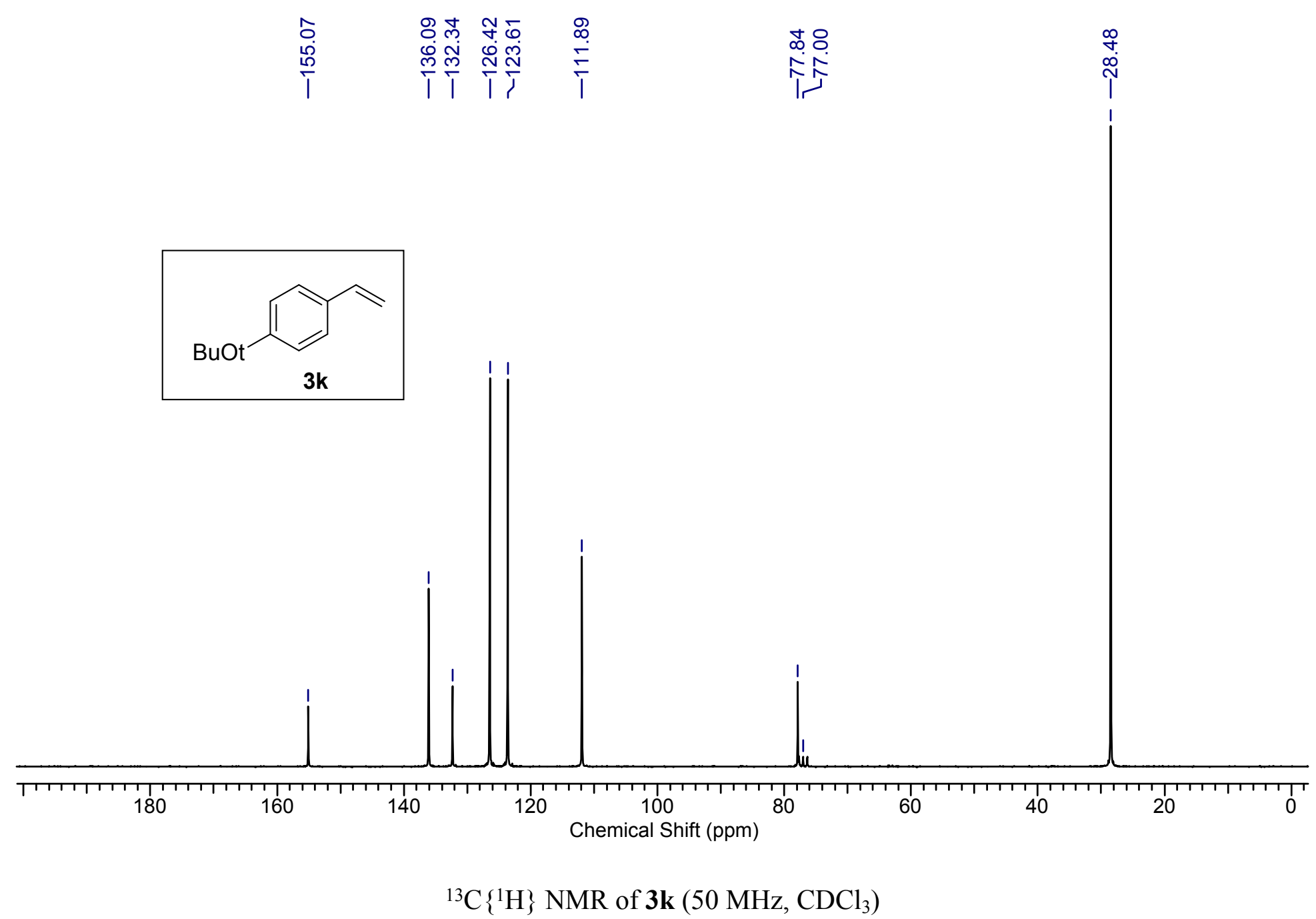




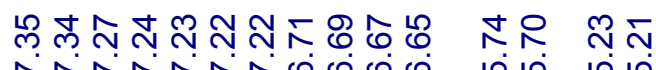

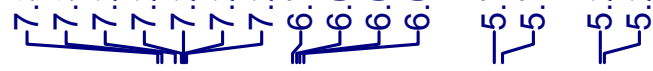
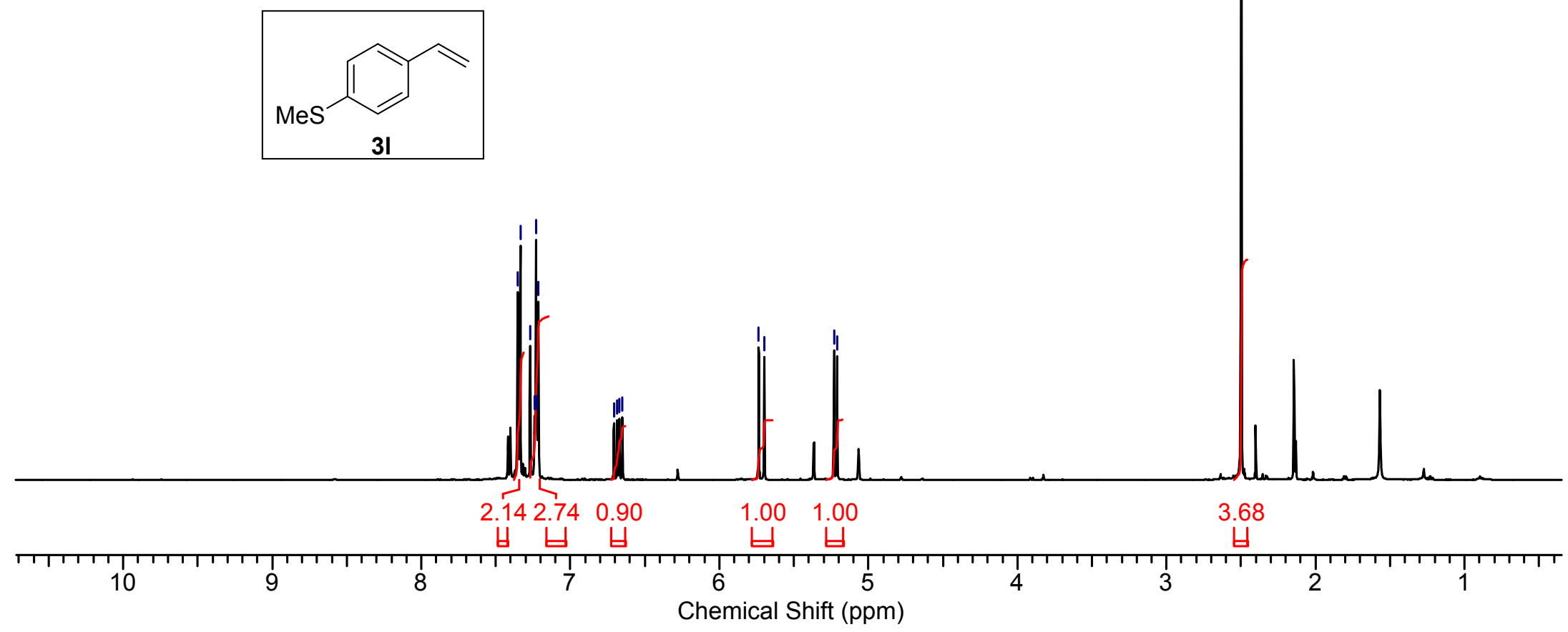

${ }^{1} \mathrm{H}$ NMR of 31 (500 MHz, $\left.\mathrm{CDCl}_{3}\right)$ 


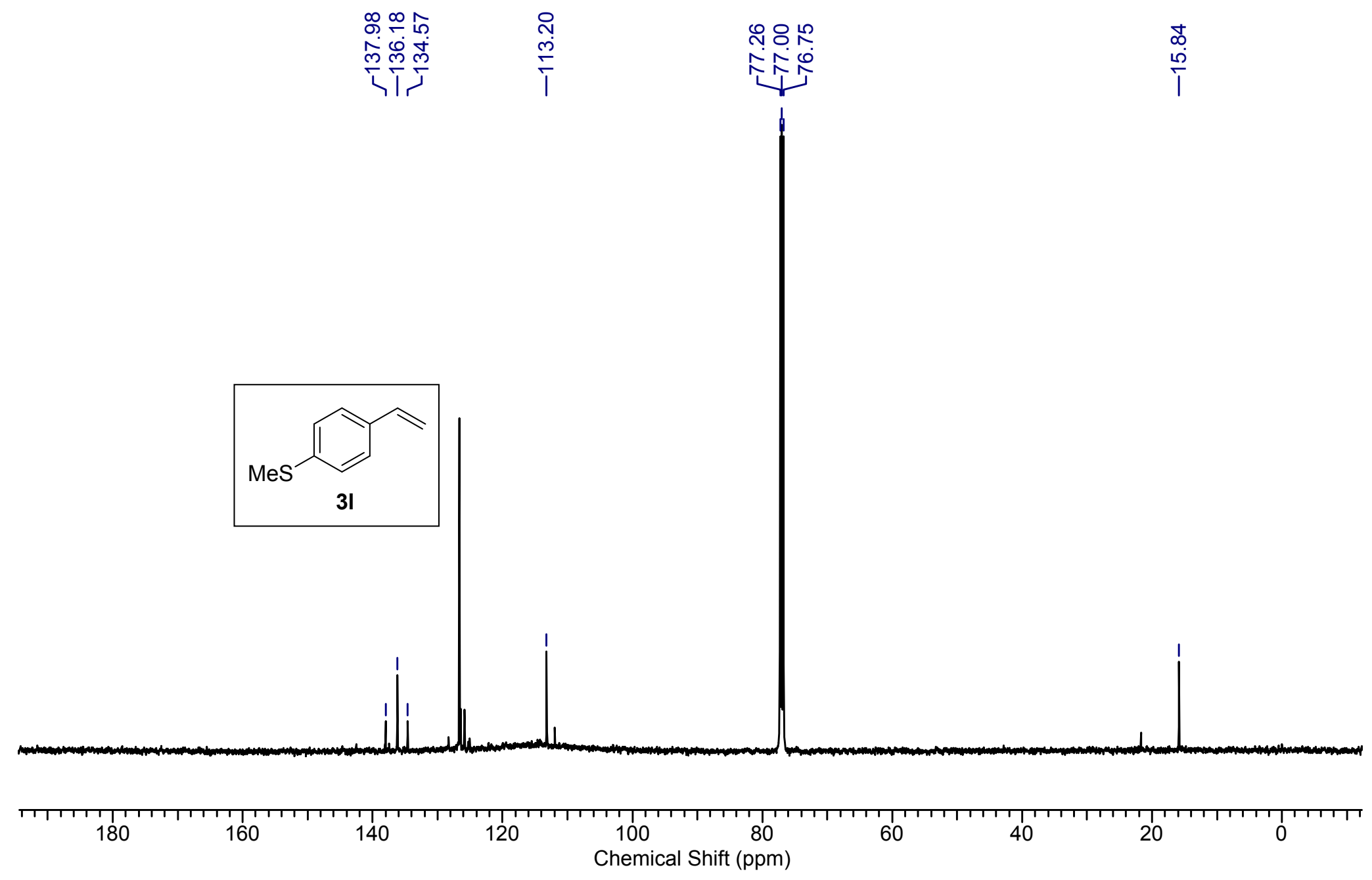

${ }^{13} \mathrm{C}\left\{{ }^{1} \mathrm{H}\right\}$ NMR of 31 (126 MHz, $\left.\mathrm{CDCl}_{3}\right)$ 


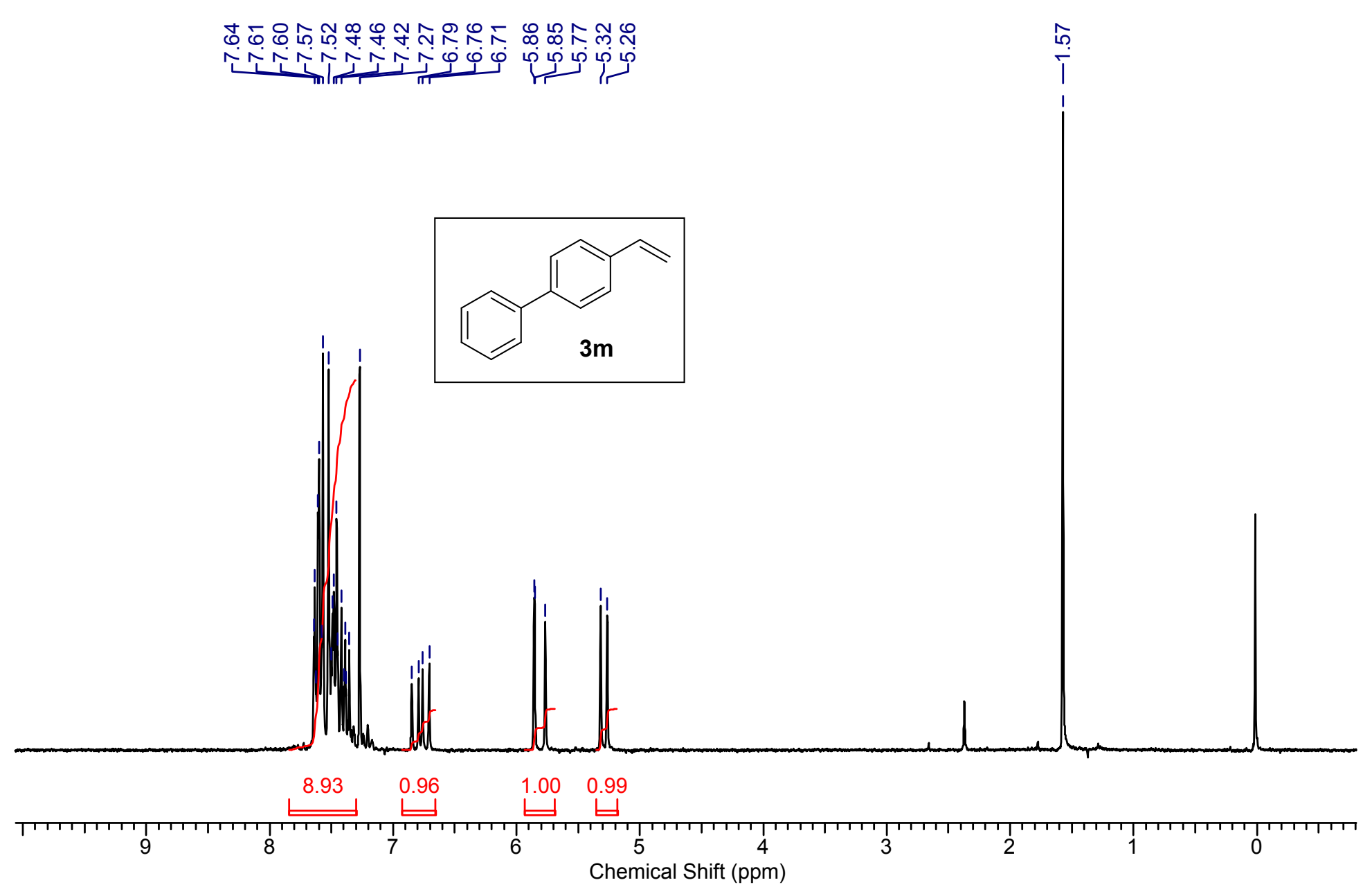

${ }^{1} \mathrm{H}$ NMR of $\mathbf{3 m}\left(200 \mathrm{MHz}, \mathrm{CDCl}_{3}\right)$ 


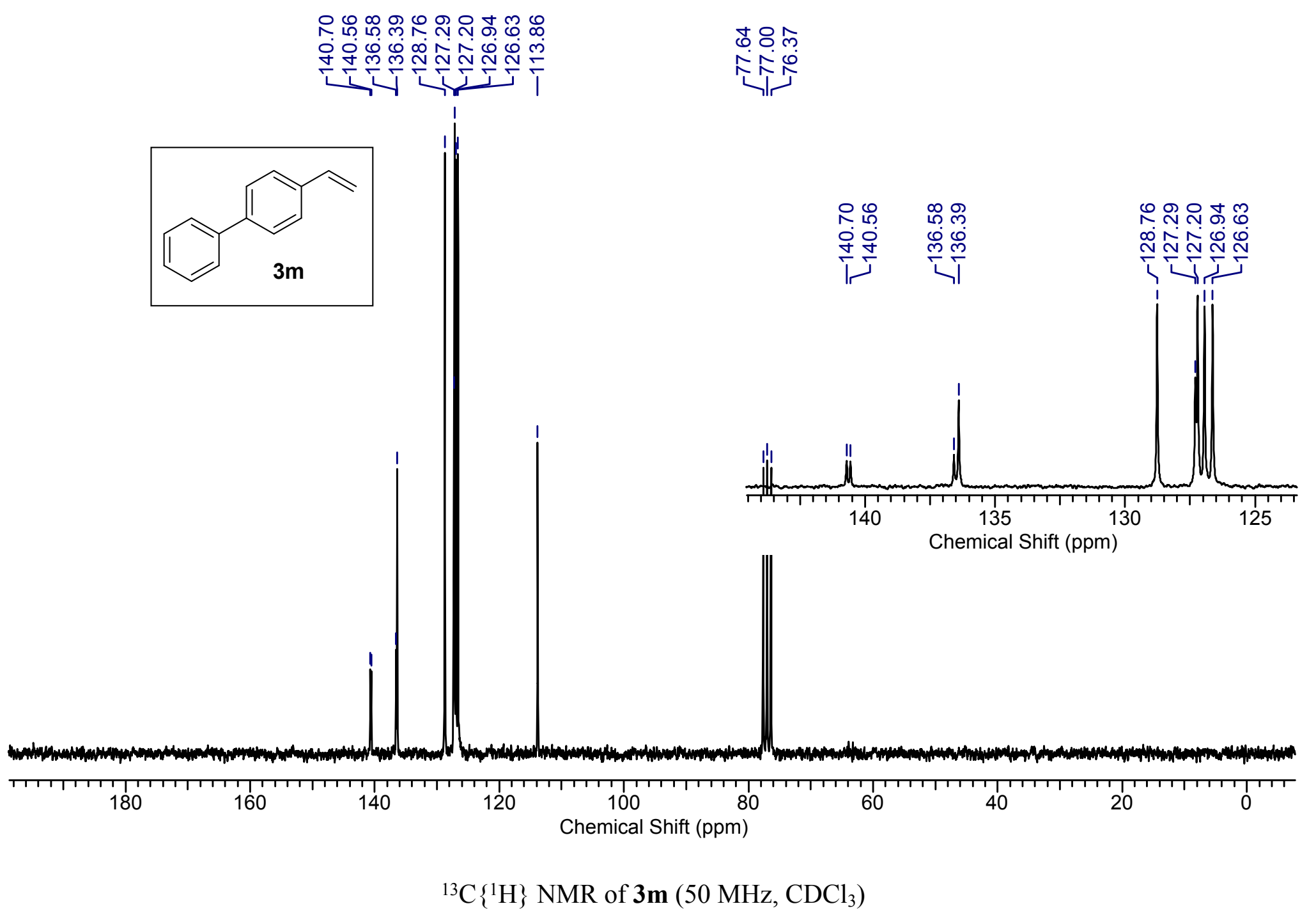




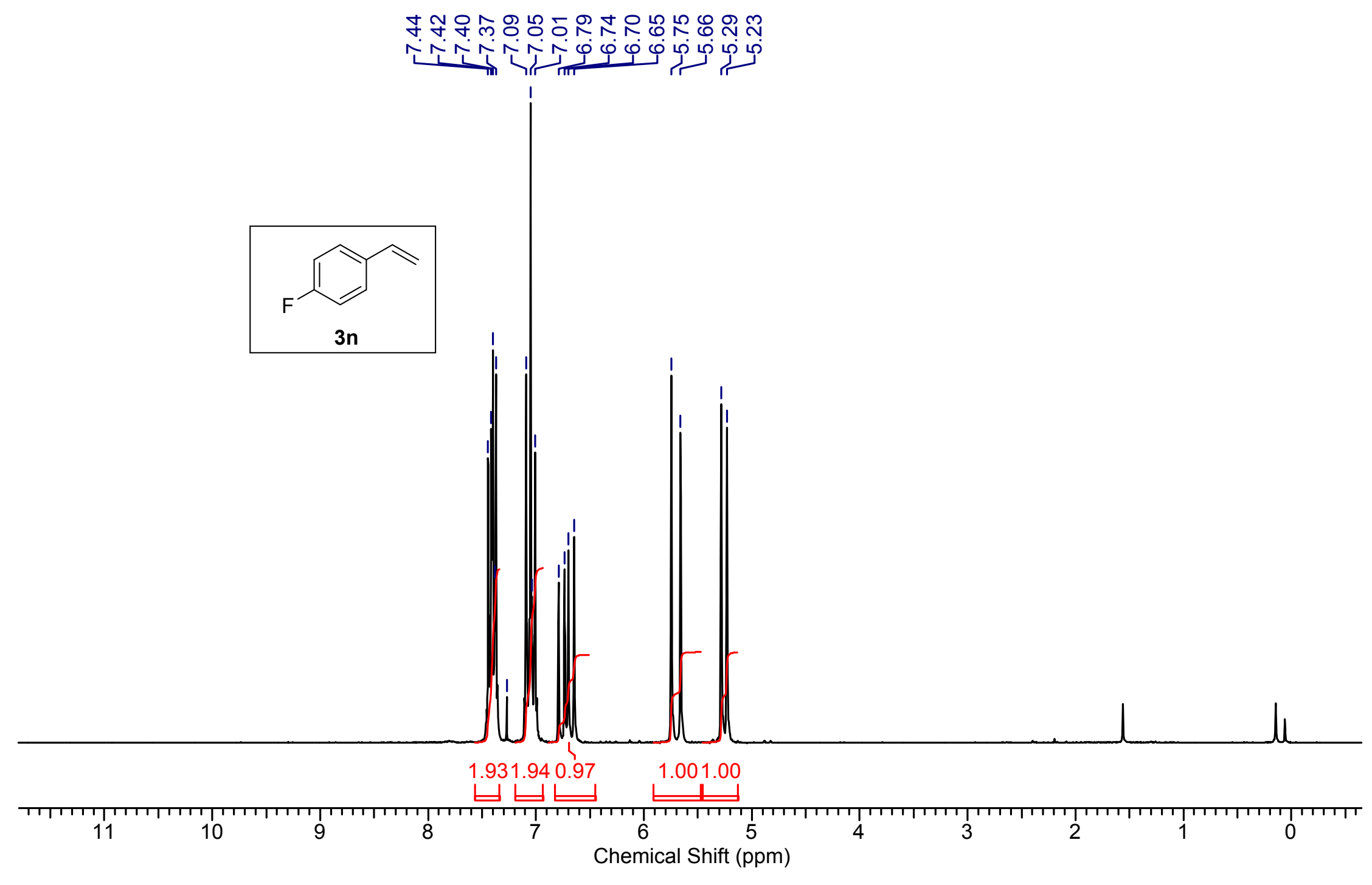

${ }^{1} \mathrm{H}$ NMR of $3 \mathbf{n}\left(200 \mathrm{MHz}, \mathrm{CDCl}_{3}\right)$ 


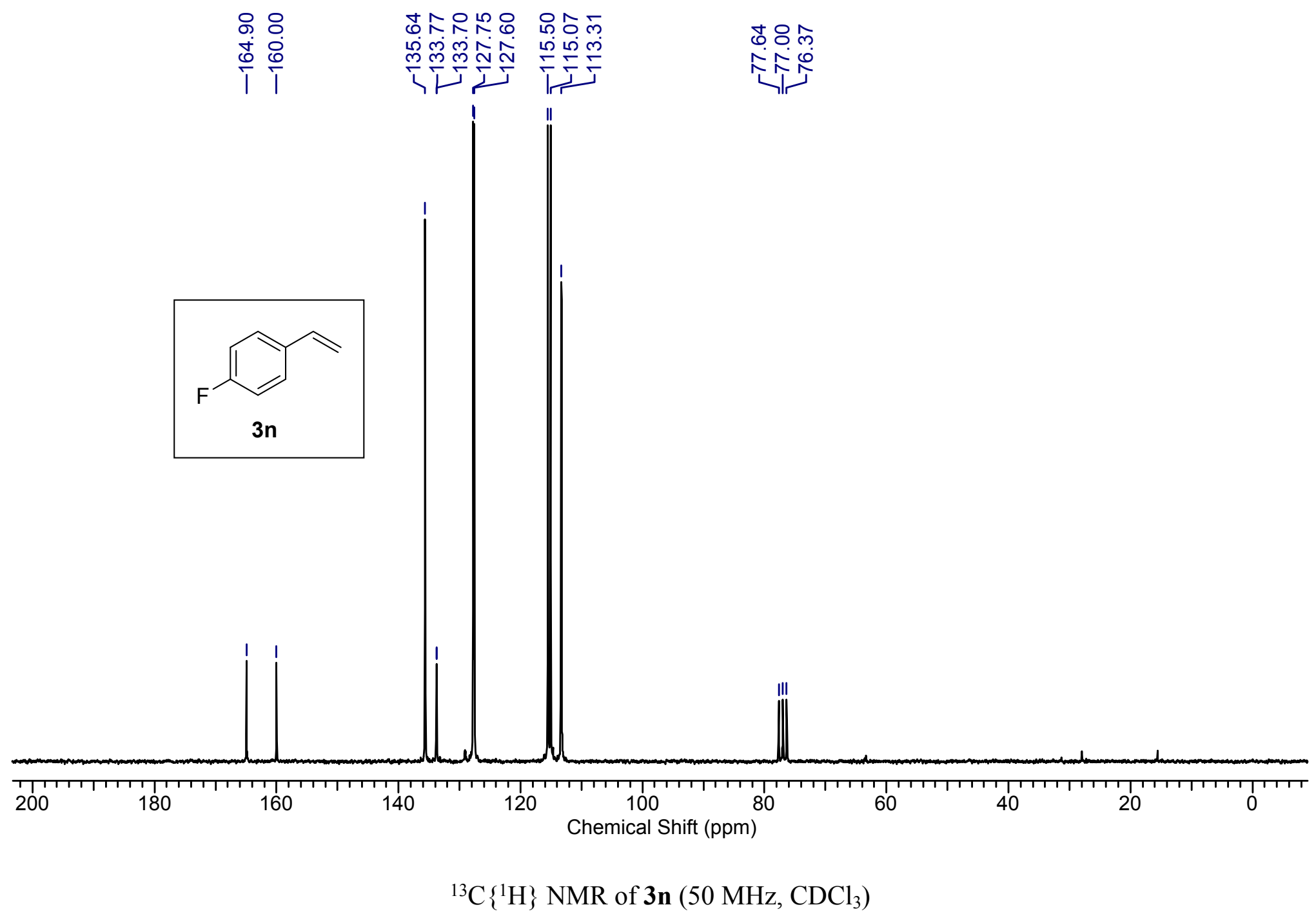




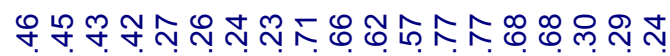

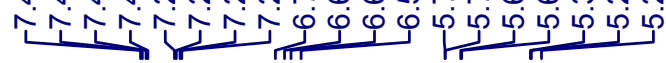

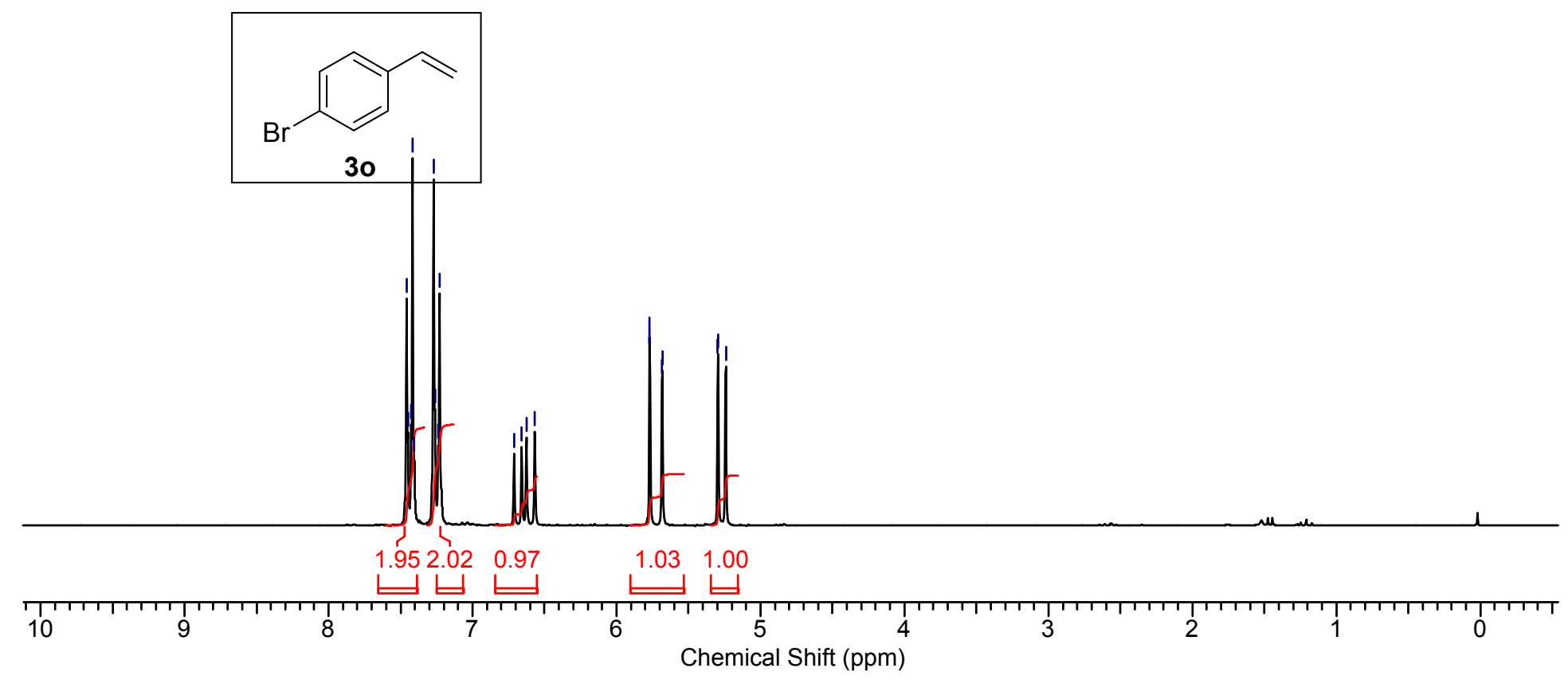

${ }^{1} \mathrm{H}$ NMR of $\mathbf{3 o}\left(200 \mathrm{MHz}, \mathrm{CDCl}_{3}\right)$ 


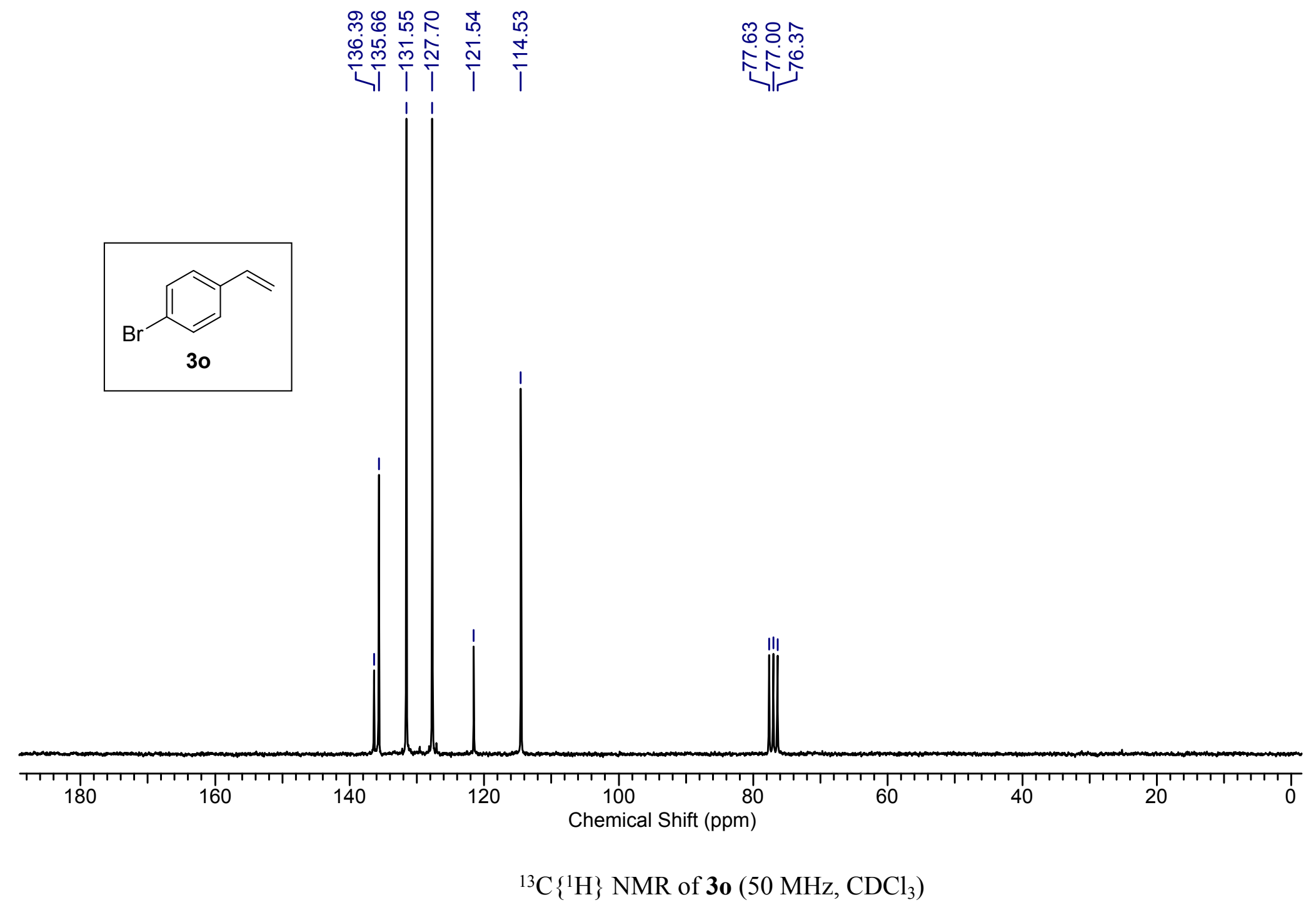




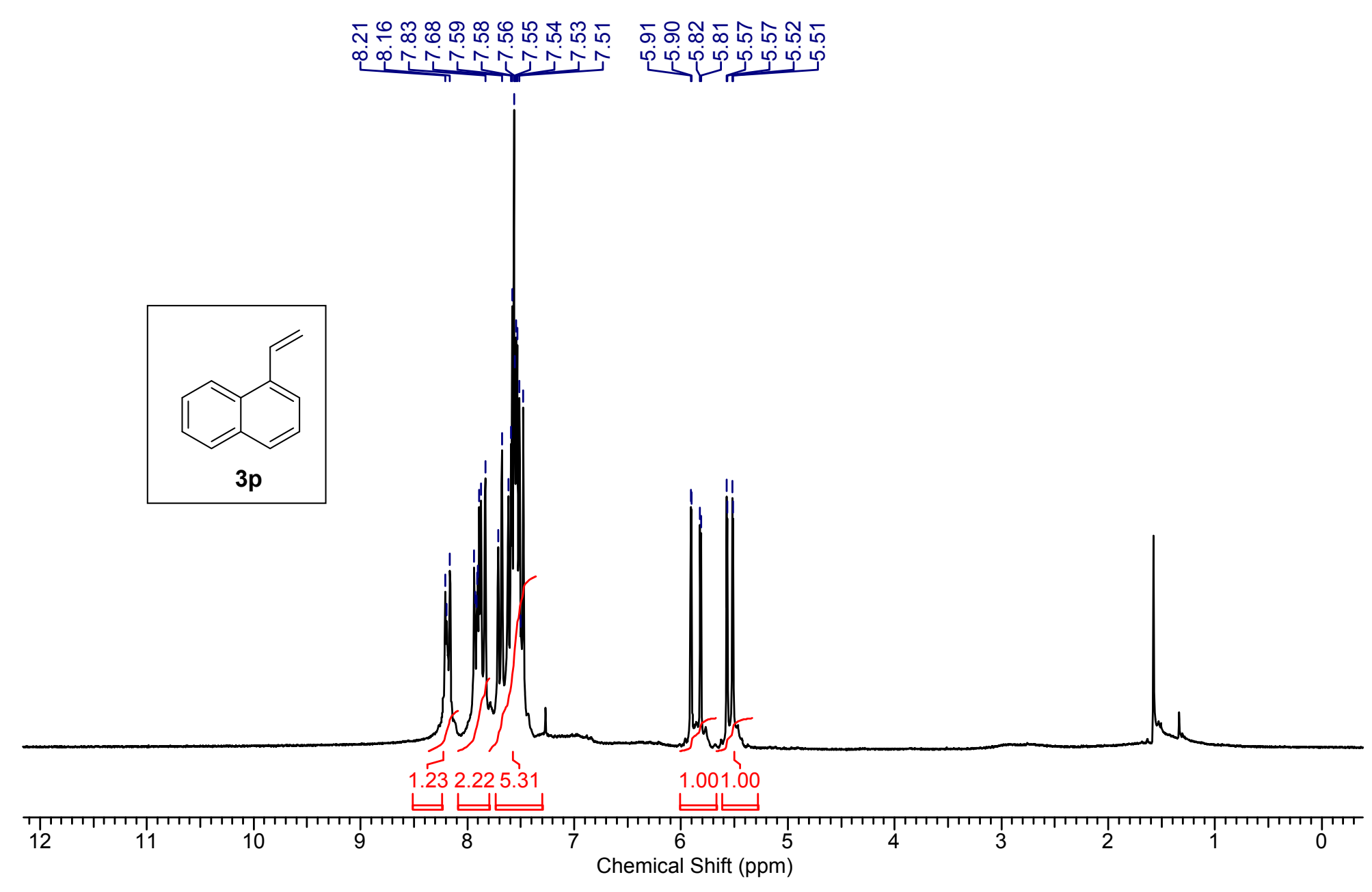

${ }^{1} \mathrm{H}$ NMR of $\mathbf{3 p}\left(200 \mathrm{MHz}, \mathrm{CDCl}_{3}\right)$ 

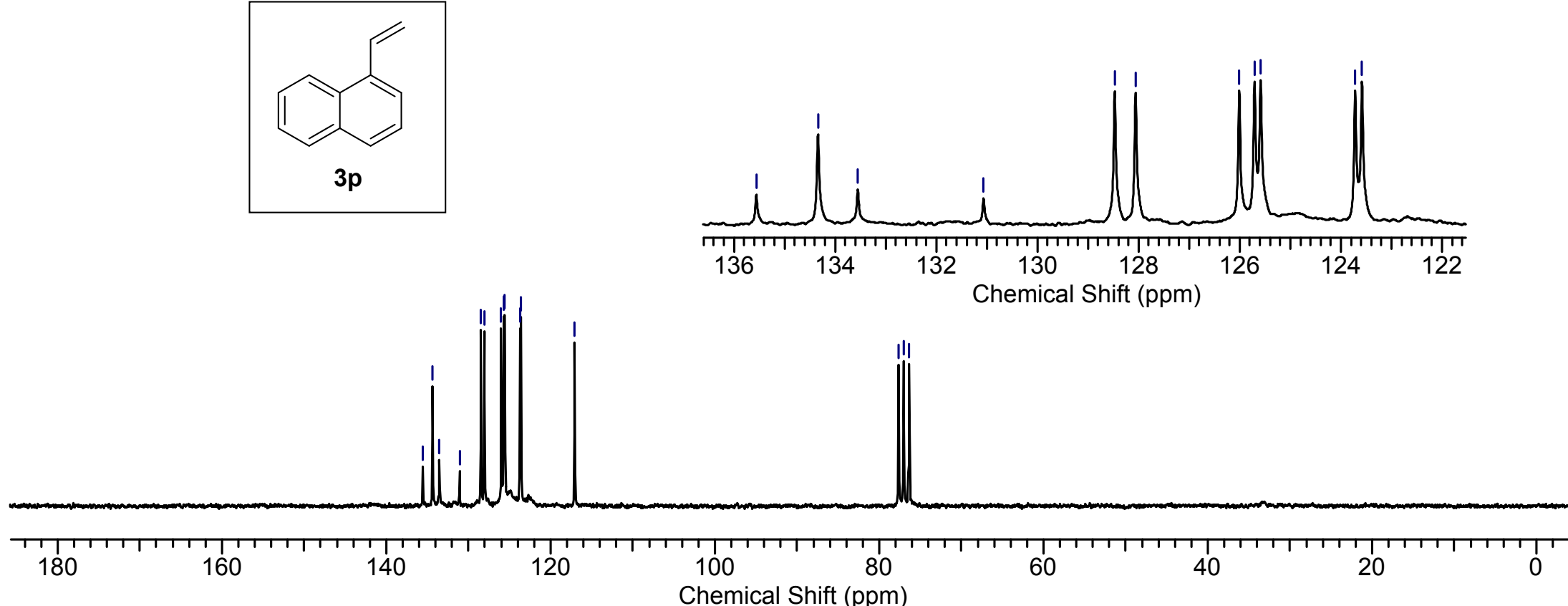

${ }^{13} \mathrm{C}\left\{{ }^{1} \mathrm{H}\right\}$ NMR of $\mathbf{3 p}\left(50 \mathrm{MHz}, \mathrm{CDCl}_{3}\right)$ 


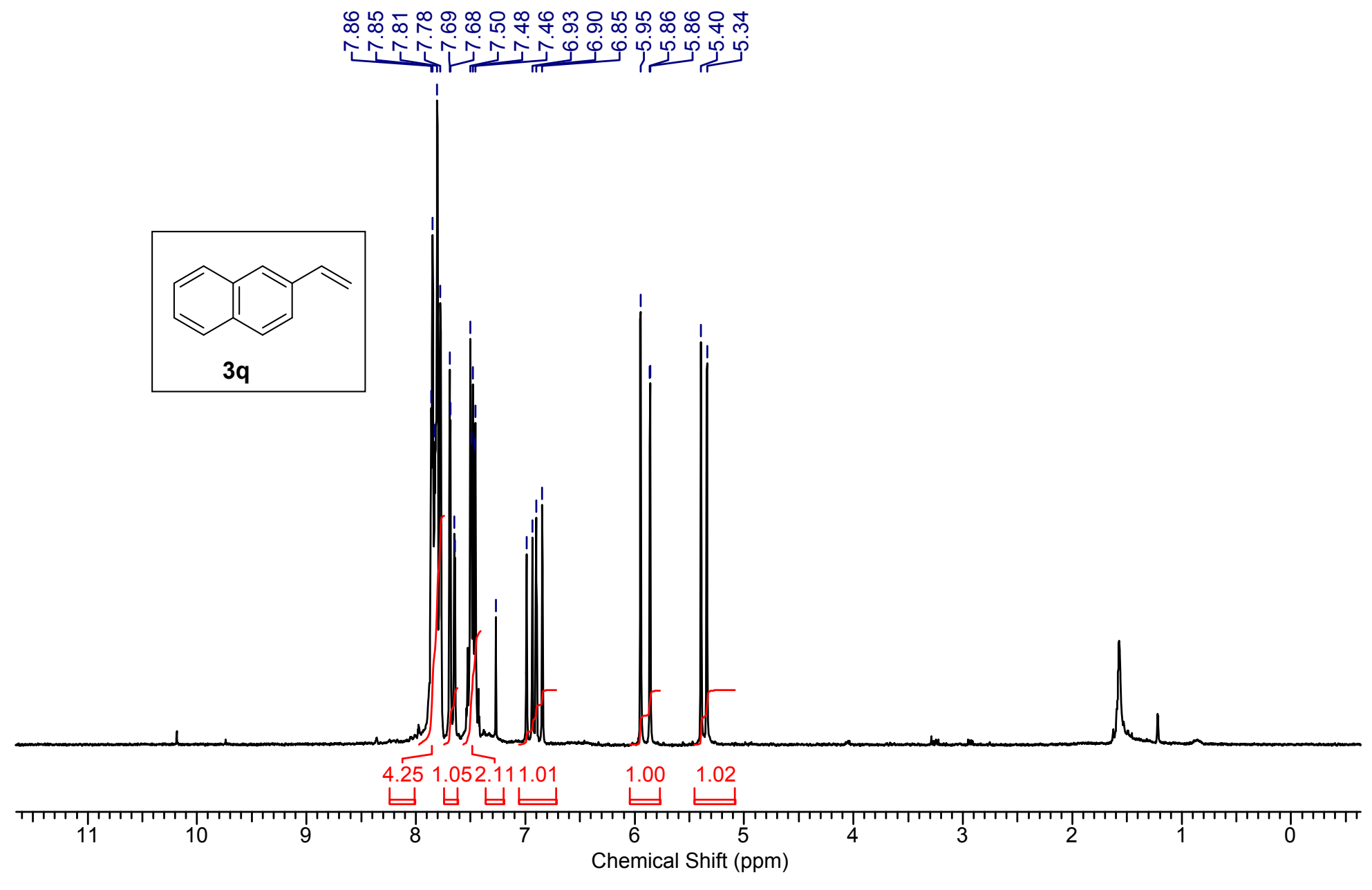

${ }^{1} \mathrm{H}$ NMR of 3q $\left(200 \mathrm{MHz}, \mathrm{CDCl}_{3}\right)$ 

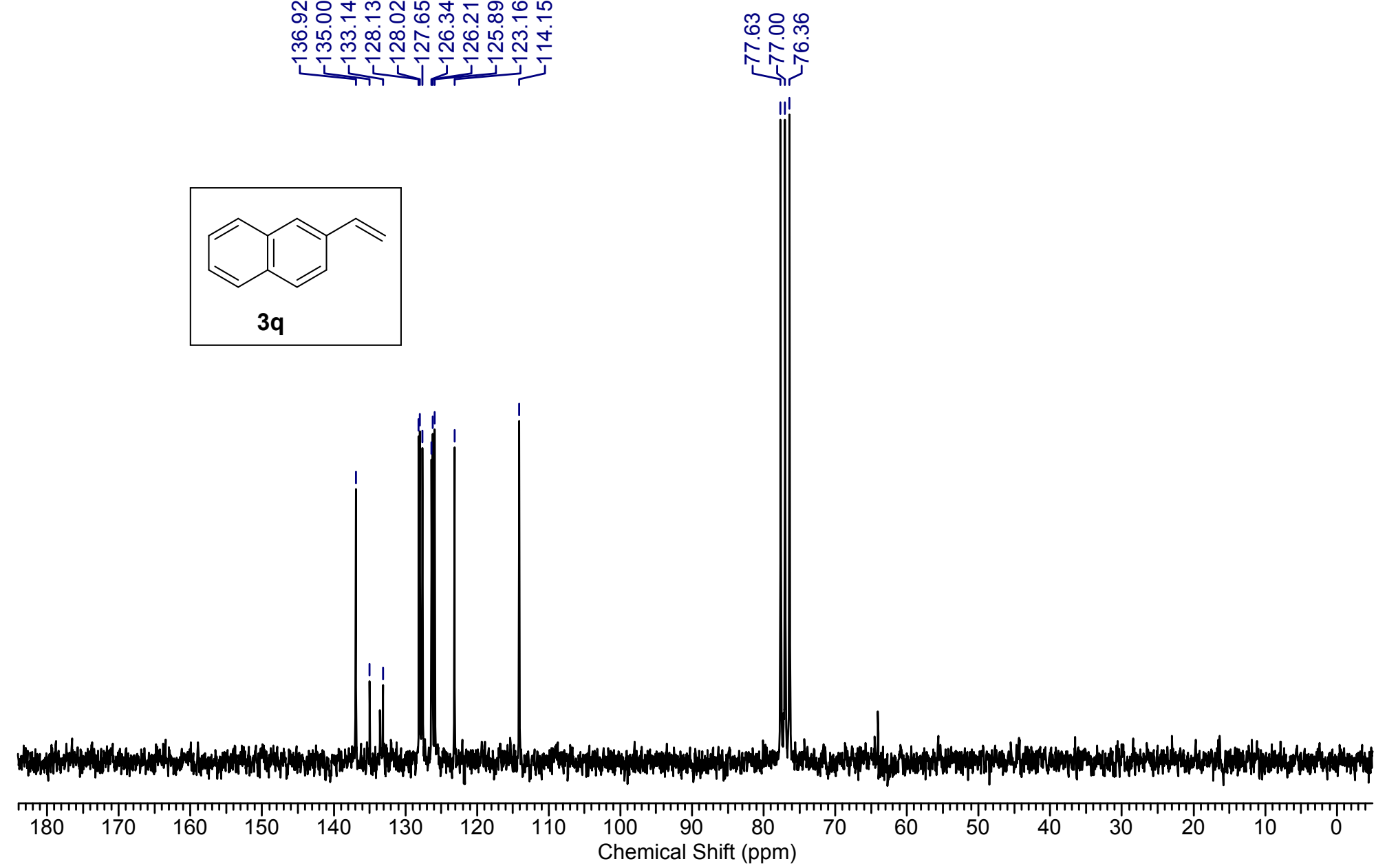

${ }^{13} \mathrm{C}\left\{{ }^{1} \mathrm{H}\right\} \mathrm{NMR}$ of $\mathbf{3 q}\left(50 \mathrm{MHz}, \mathrm{CDCl}_{3}\right)$ 


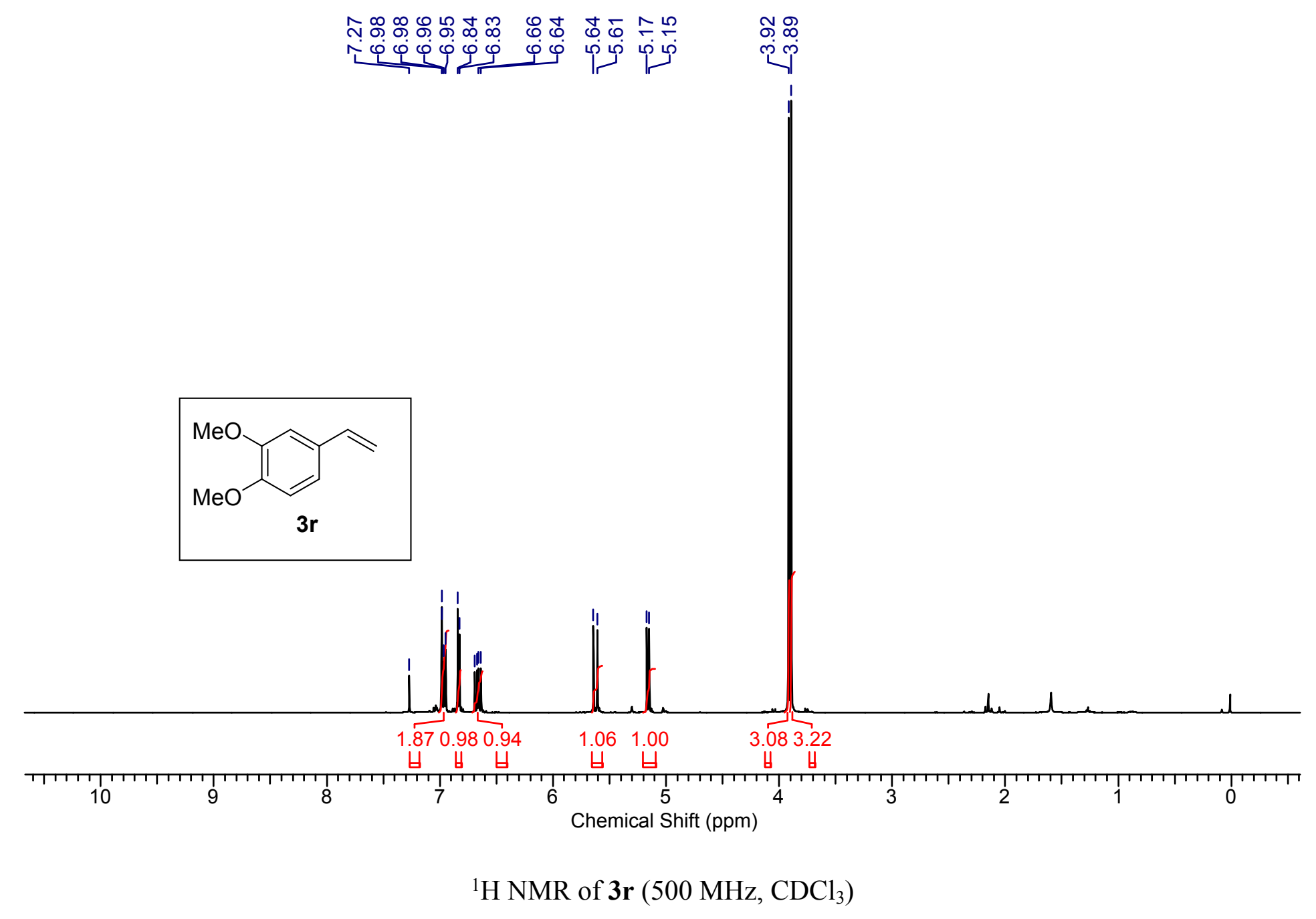




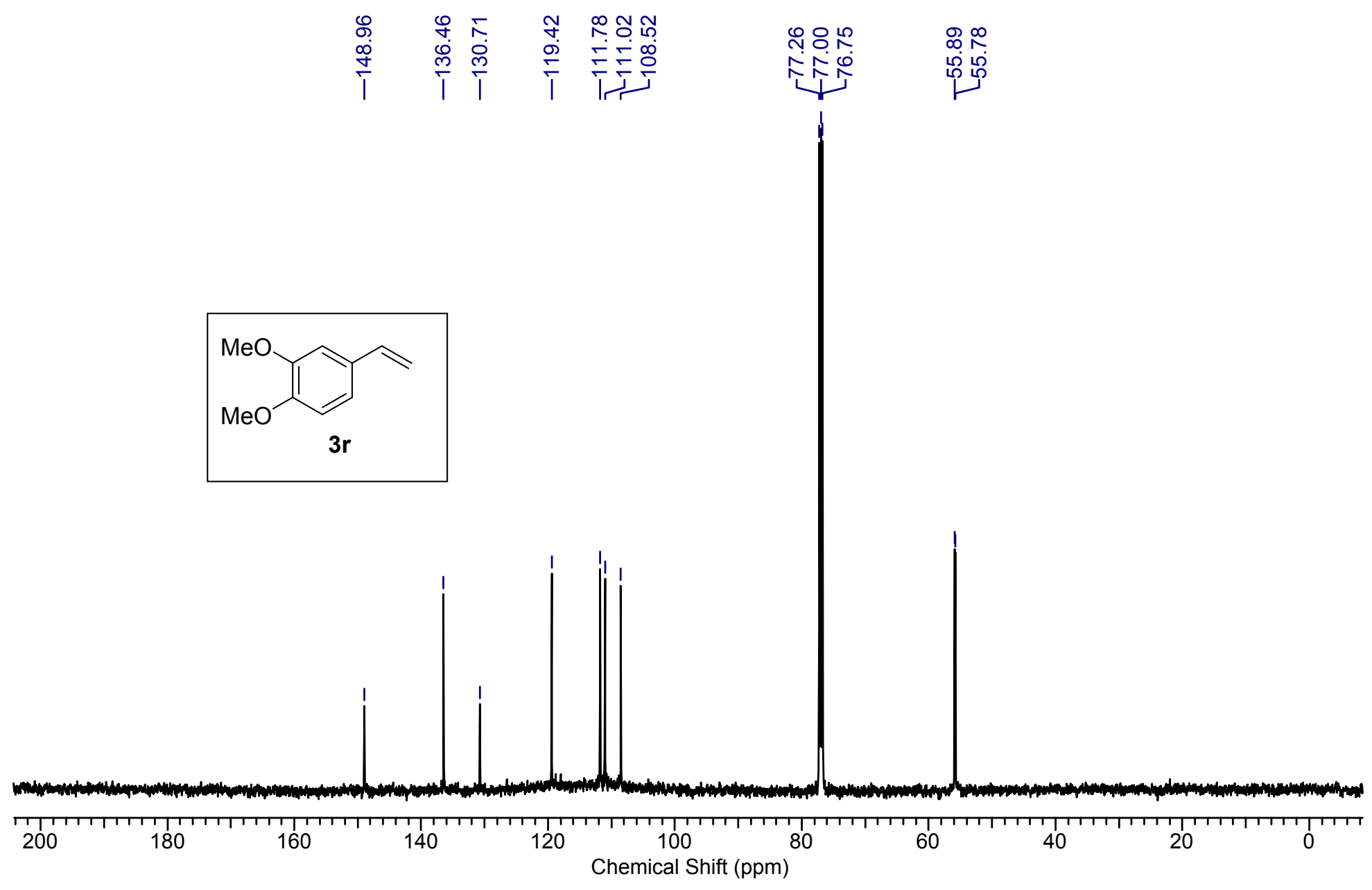

${ }^{13} \mathrm{C}\left\{{ }^{1} \mathrm{H}\right\} \mathrm{NMR}$ of $3 \mathbf{r}\left(126 \mathrm{MHz}, \mathrm{CDCl}_{3}\right)$ 


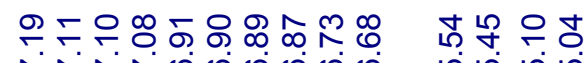

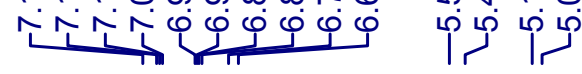

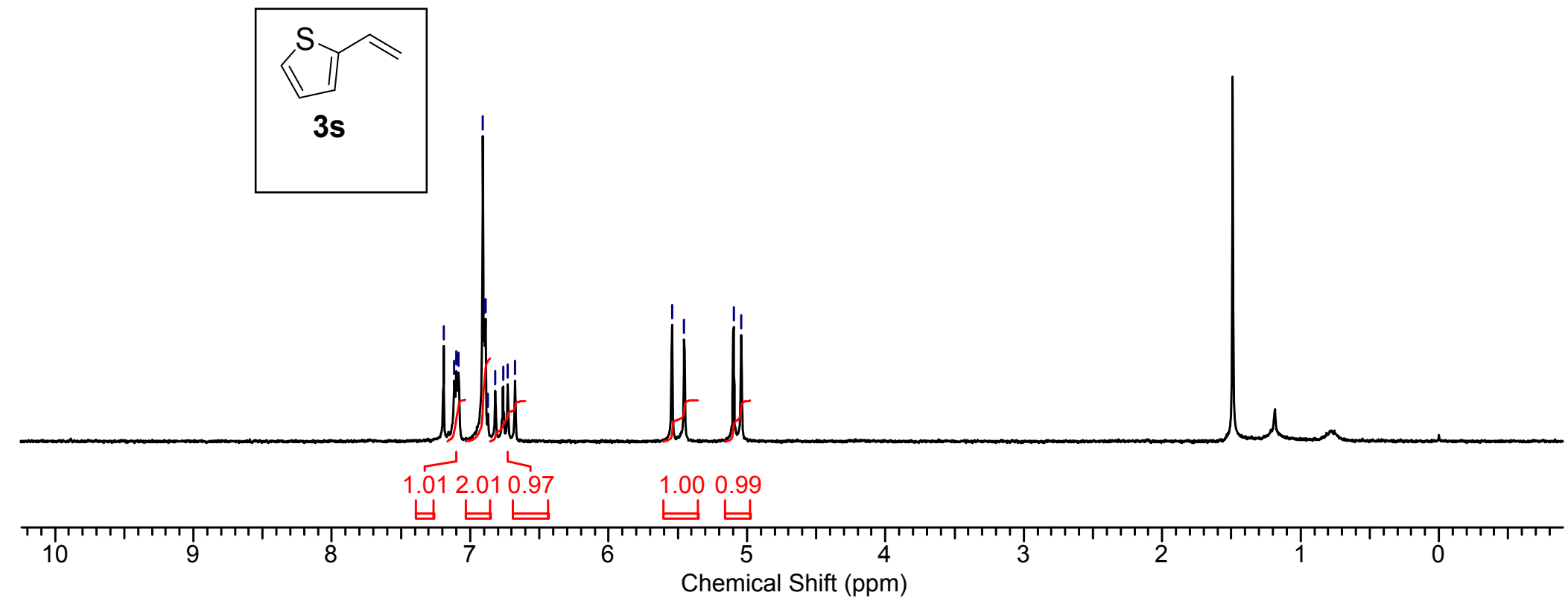

${ }^{1} \mathrm{H}$ NMR of $3 \mathbf{s}\left(200 \mathrm{MHz}, \mathrm{CDCl}_{3}\right)$ 


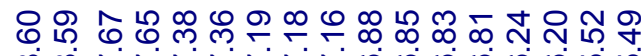

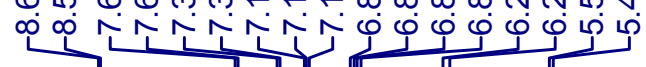

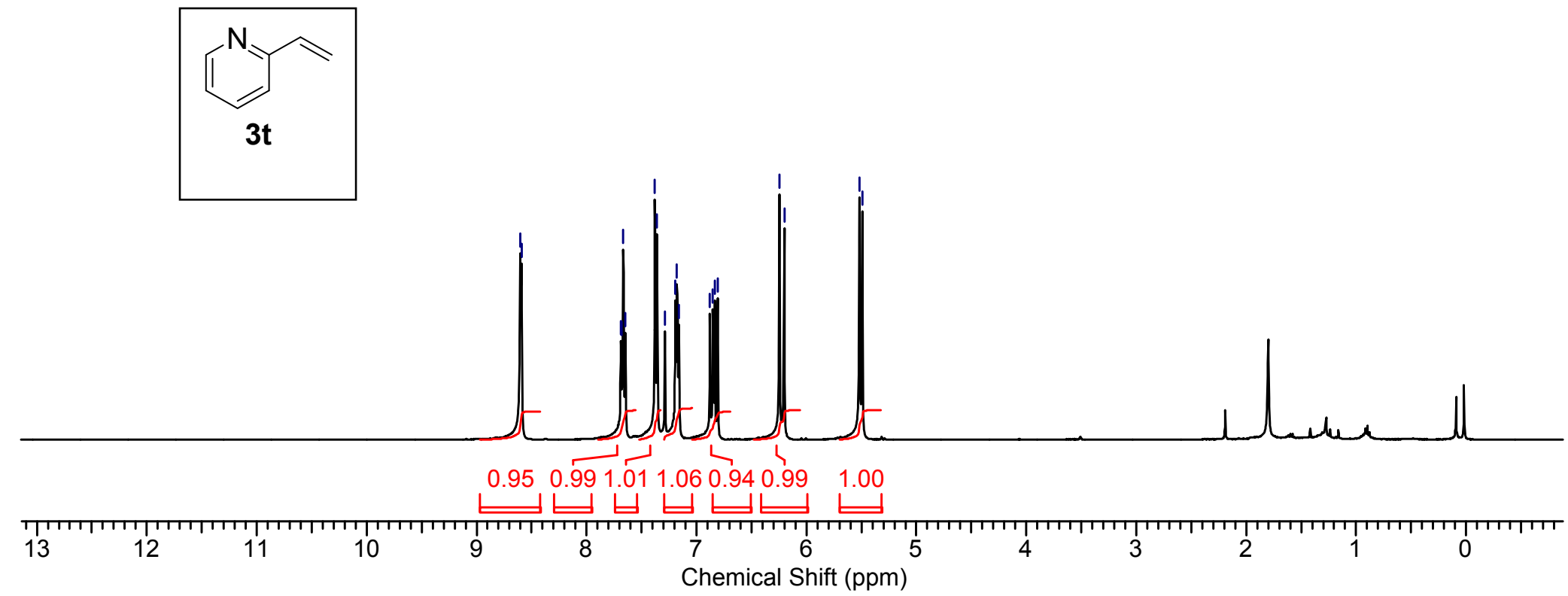

${ }^{1} \mathrm{H}$ NMR of $\mathbf{3 t}\left(400 \mathrm{MHz}, \mathrm{CDCl}_{3}\right)$ 


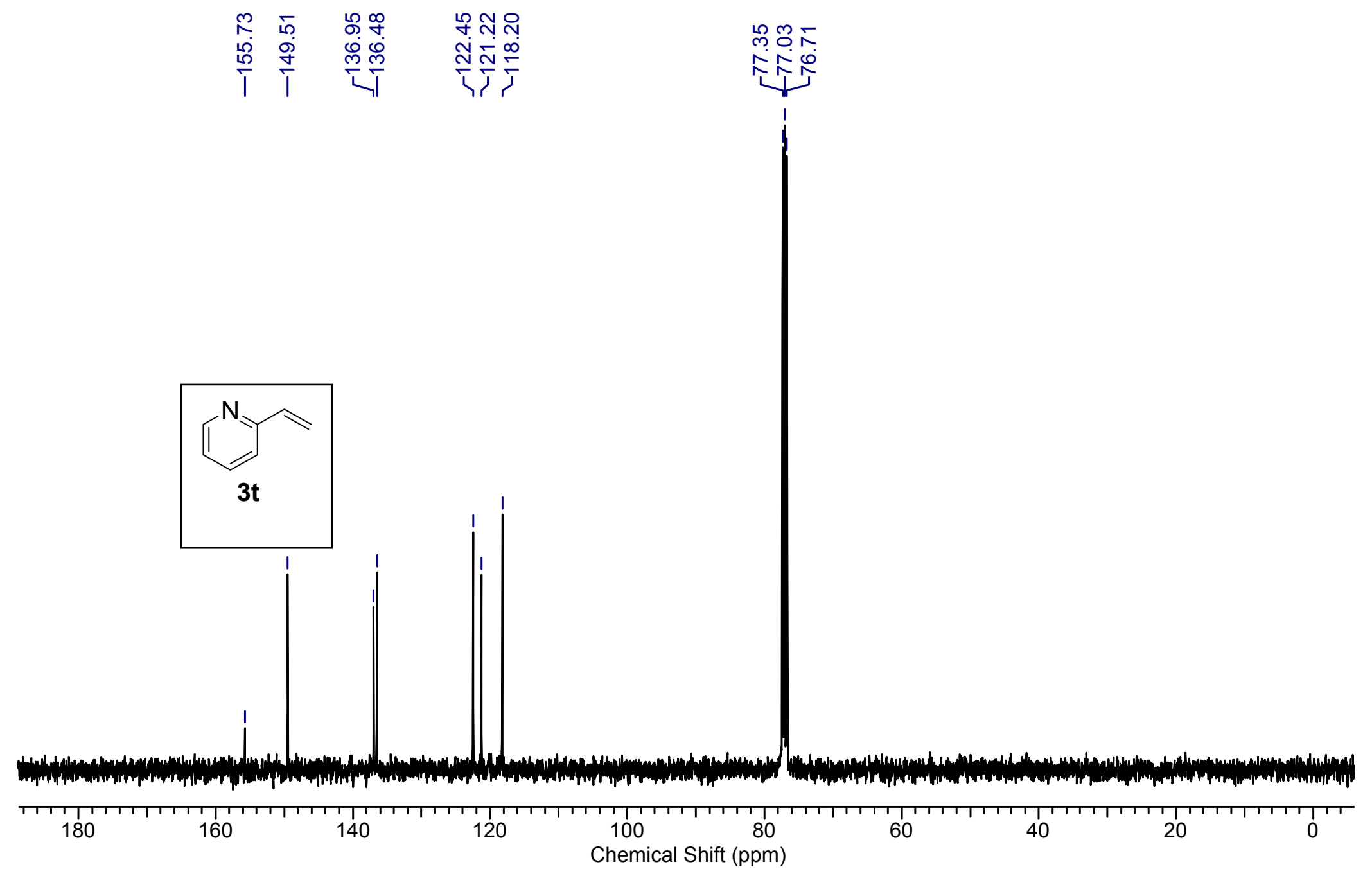

${ }^{13} \mathrm{C}\left\{{ }^{1} \mathrm{H}\right\}$ NMR of $\mathbf{3 t}\left(101 \mathrm{MHz}, \mathrm{CDCl}_{3}\right)$ 
N $\stackrel{N}{N} \stackrel{\text { N }}{4}$

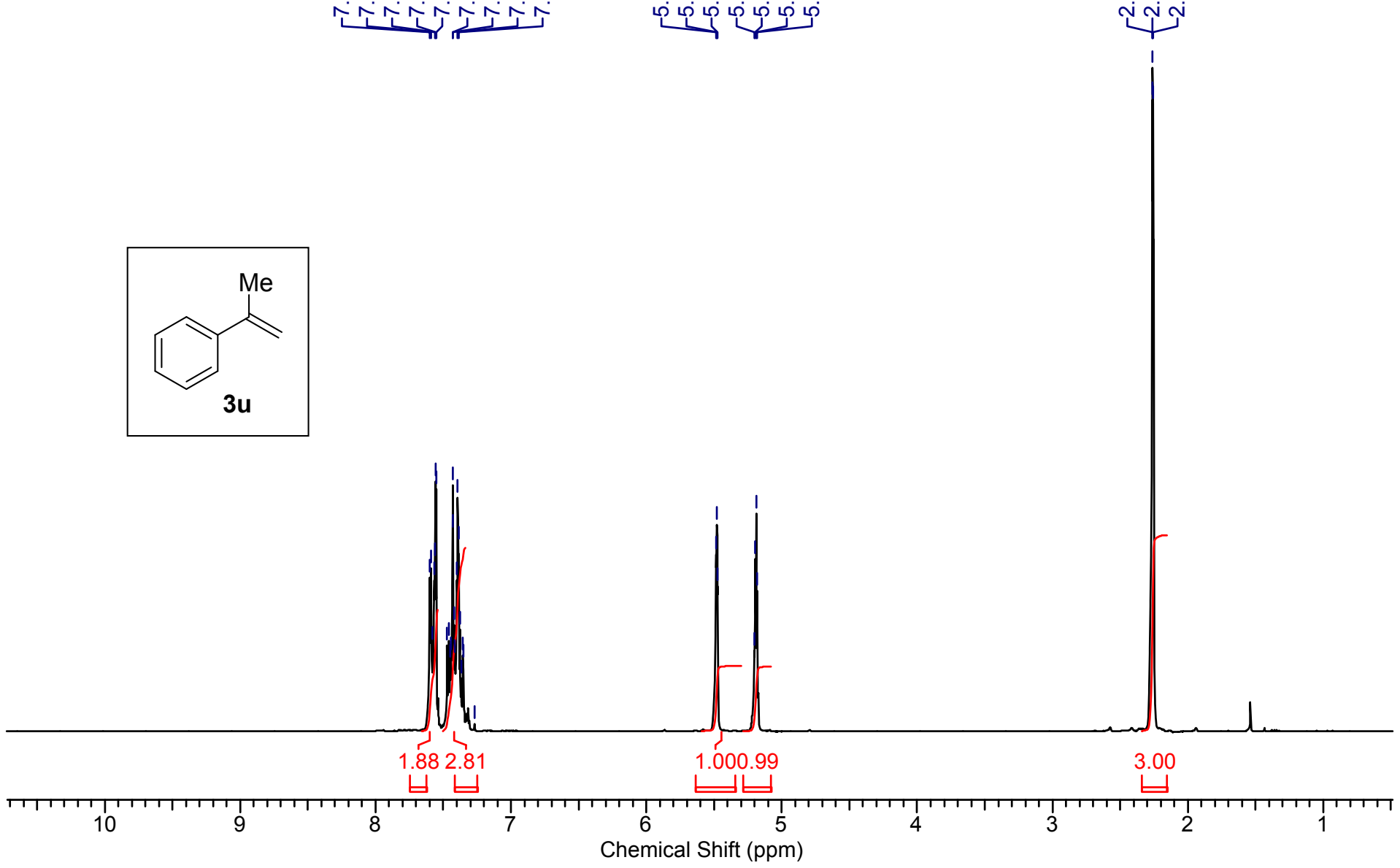

${ }^{1} \mathrm{H}$ NMR of $3 \mathbf{u}\left(200 \mathrm{MHz}, \mathrm{CDCl}_{3}\right)$ 


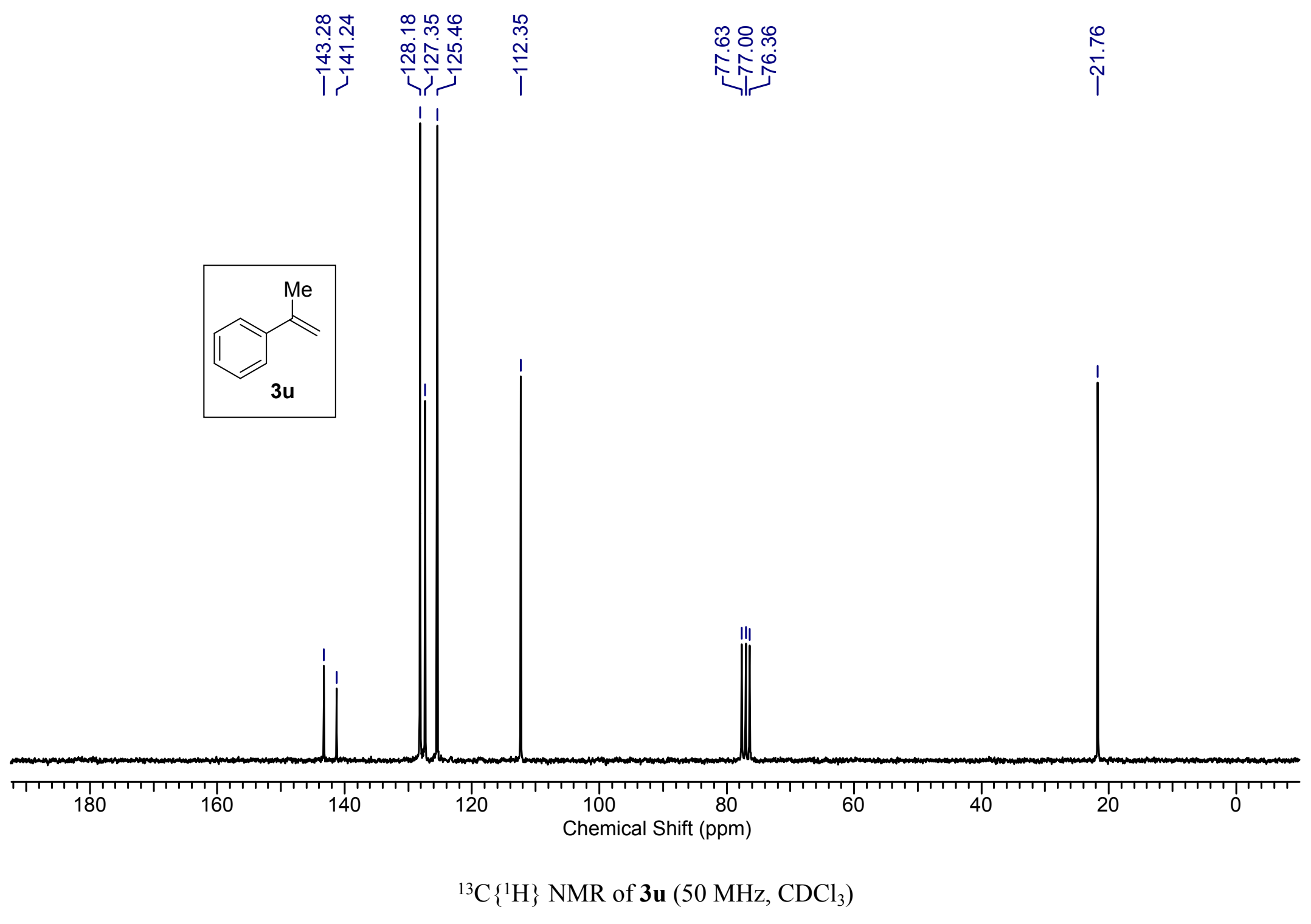




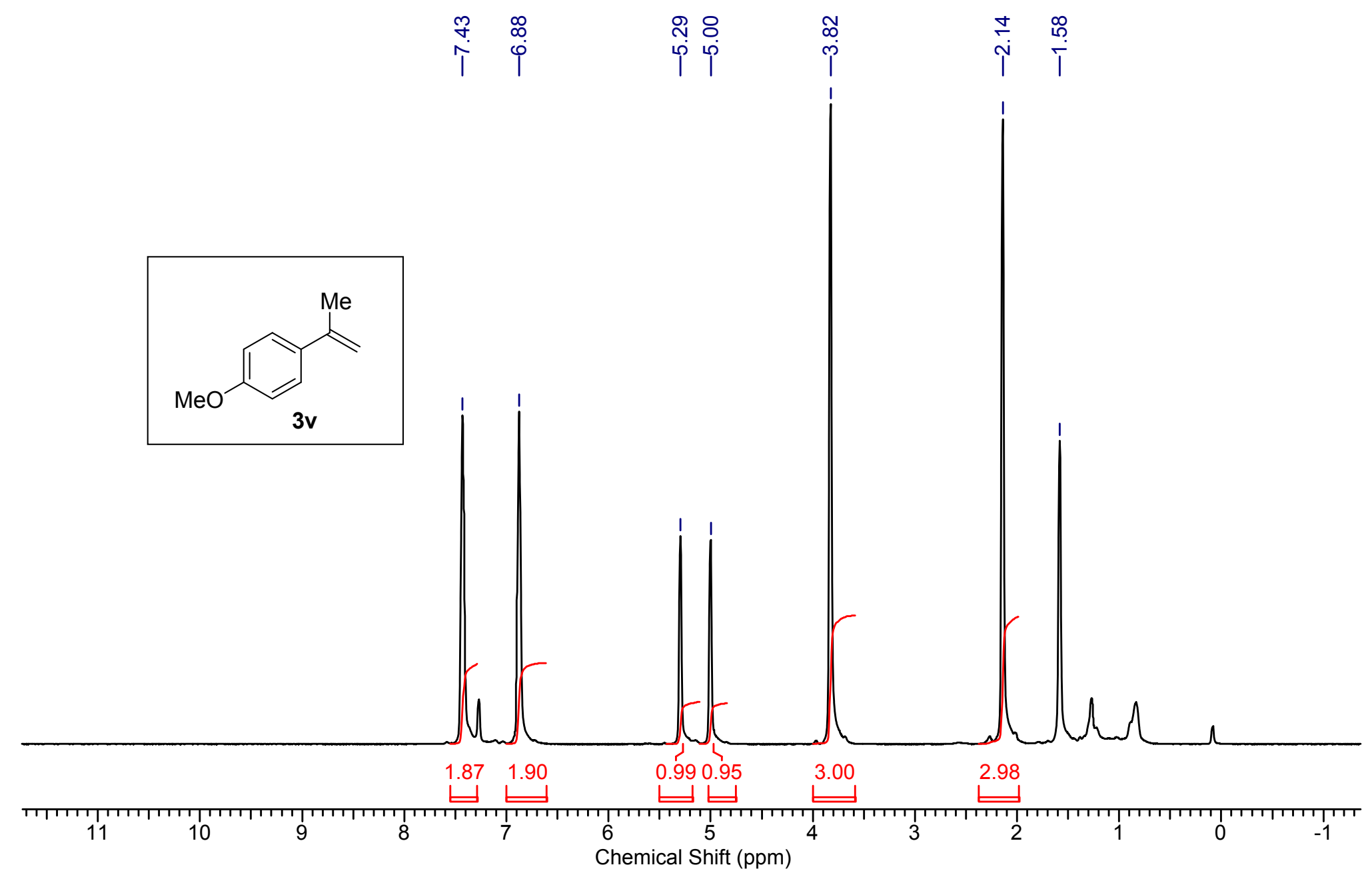

${ }^{1} \mathrm{H} \mathrm{NMR}$ of $\mathbf{3 v}\left(500 \mathrm{MHz}, \mathrm{CDCl}_{3}\right)$ 


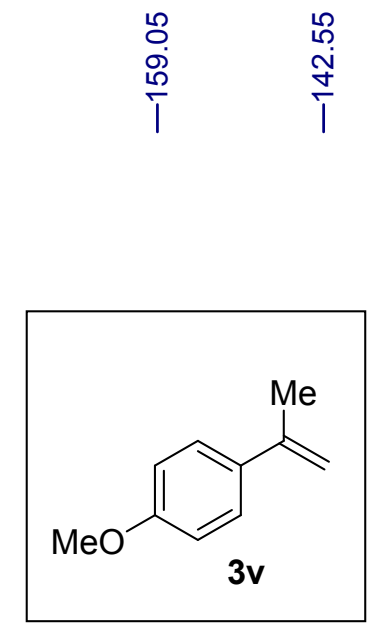

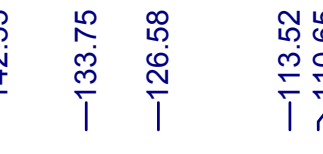

产芹

นุำ

숭

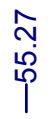

$\stackrel{8}{i}$
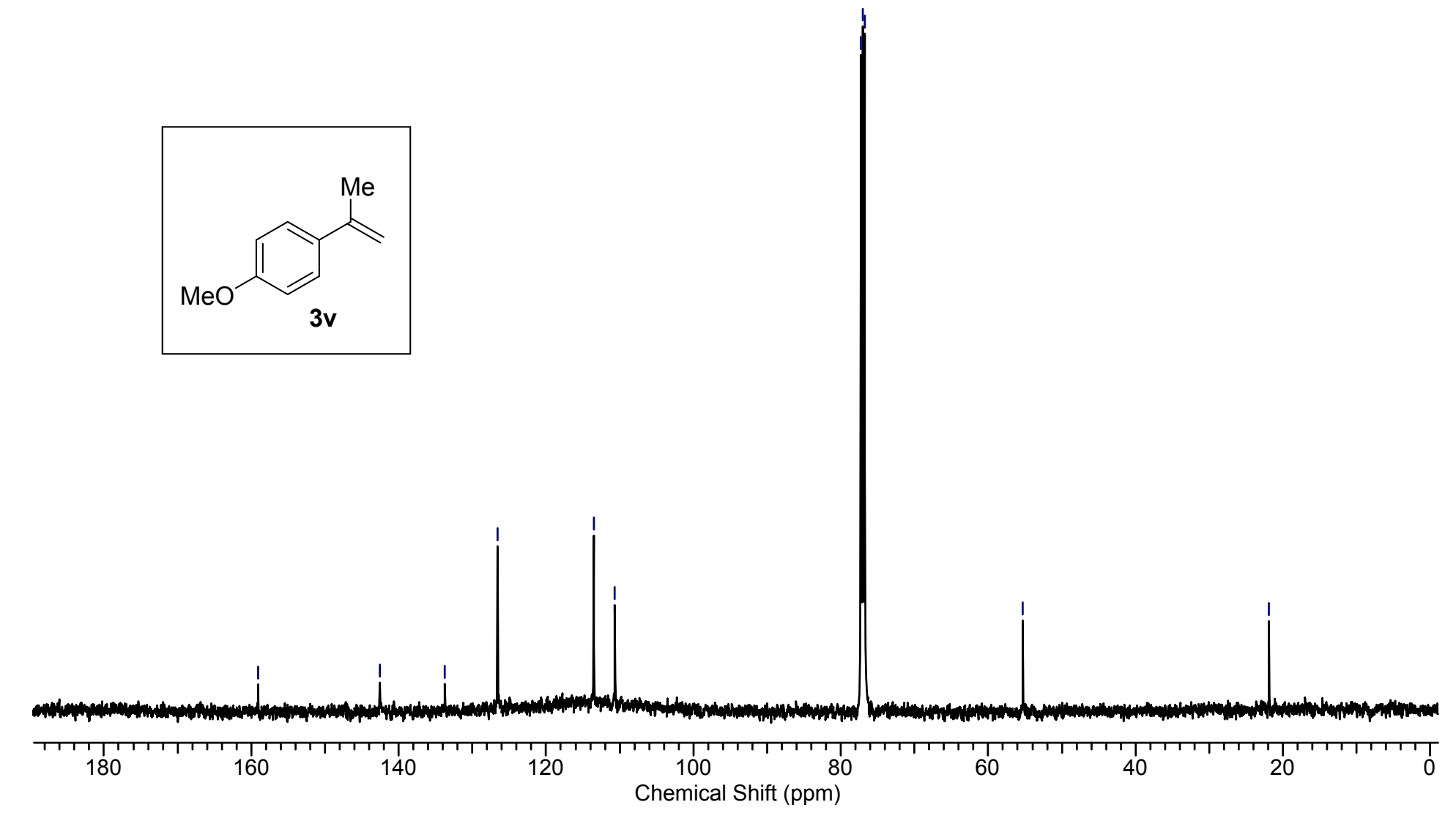

${ }^{13} \mathrm{C}\left\{{ }^{1} \mathrm{H}\right\}$ NMR of $3 \mathbf{v}\left(126 \mathrm{MHz}, \mathrm{CDCl}_{3}\right)$ 

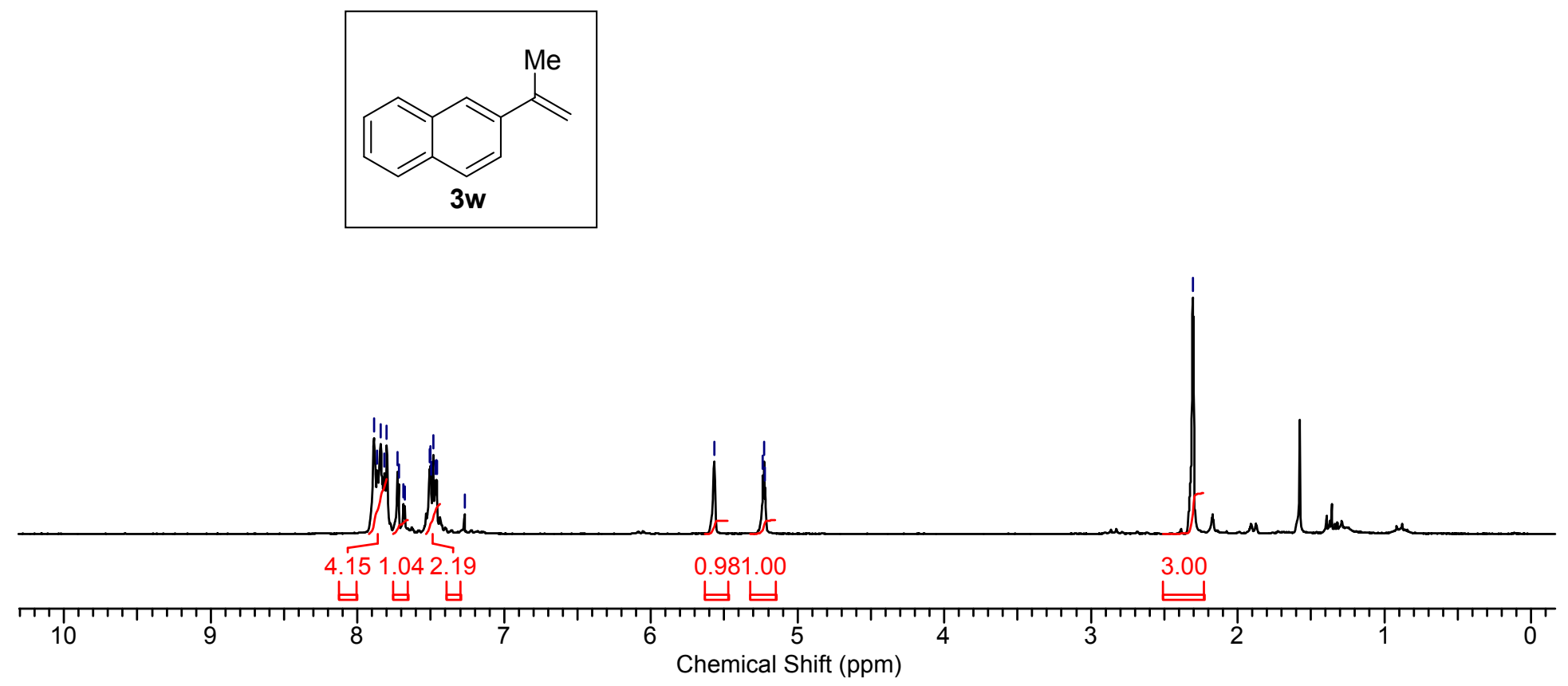

${ }^{1} \mathrm{H}$ NMR of $\mathbf{3 w}\left(200 \mathrm{MHz}, \mathrm{CDCl}_{3}\right)$ 

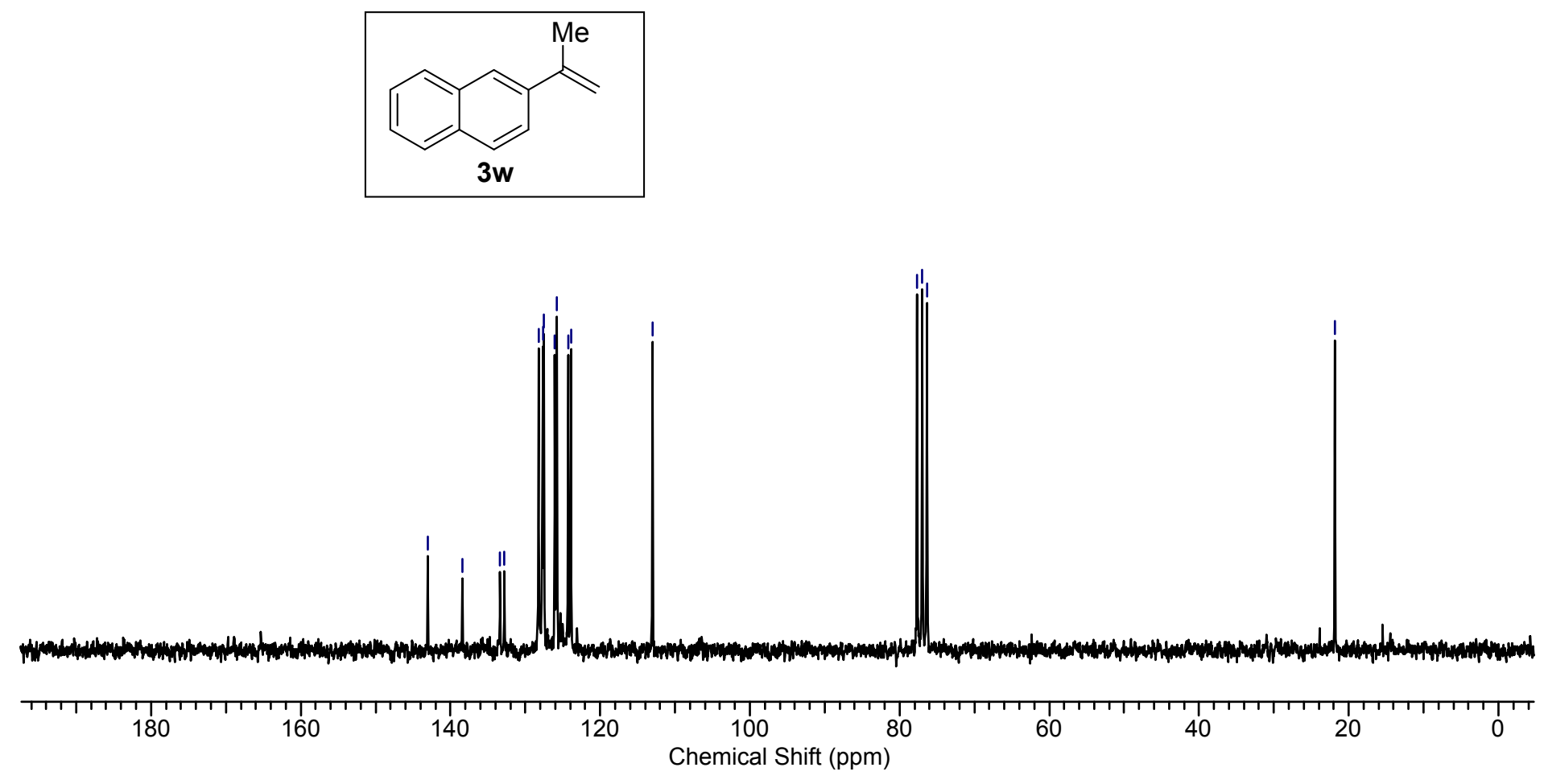

${ }^{13} \mathrm{C}\left\{{ }^{1} \mathrm{H}\right\}$ NMR of $\mathbf{3 w}\left(50 \mathrm{MHz}, \mathrm{CDCl}_{3}\right)$ 


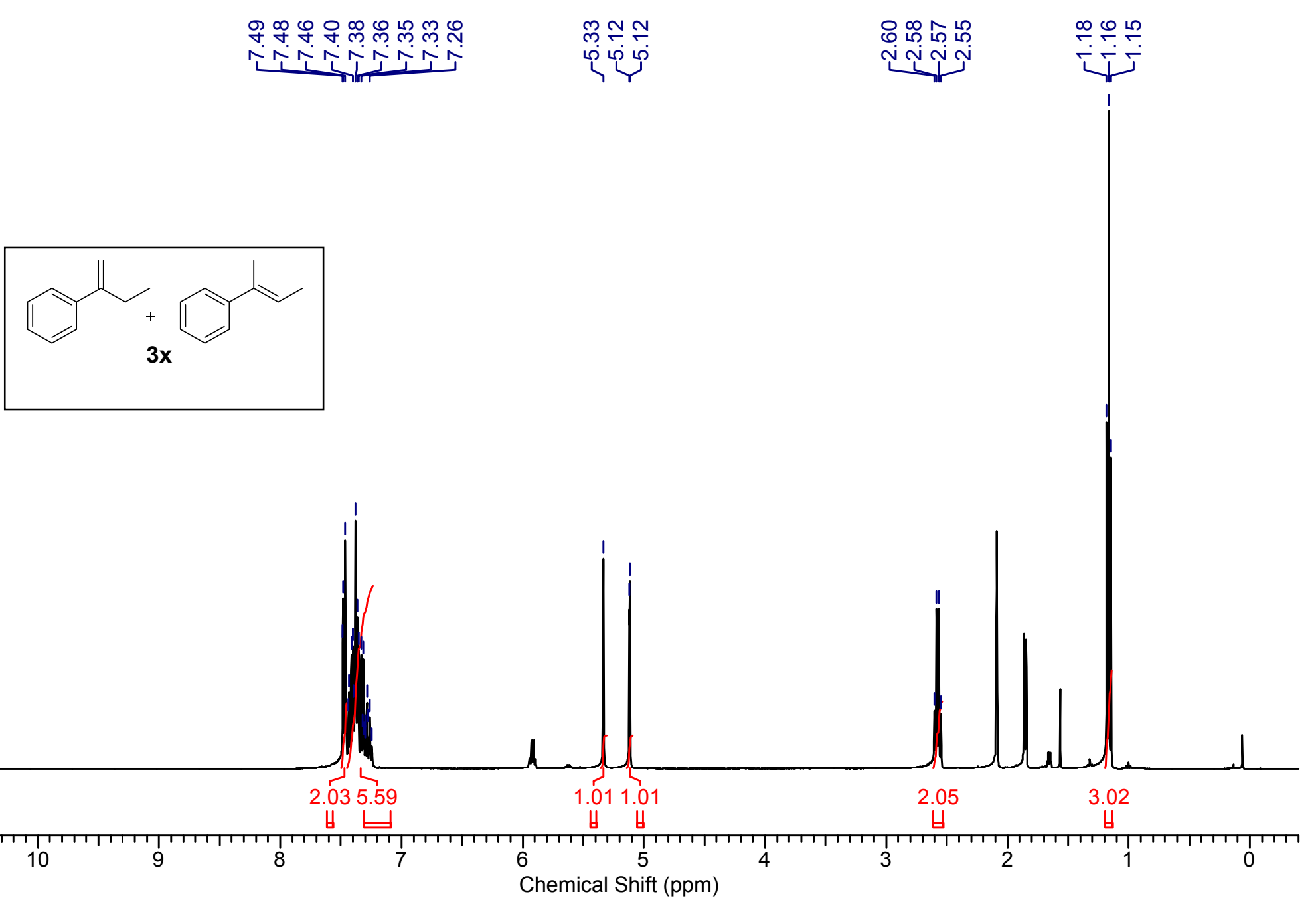

${ }^{1} \mathrm{H}$ NMR of $\mathbf{3 x}\left(400 \mathrm{MHz}, \mathrm{CDCl}_{3}\right)$ 


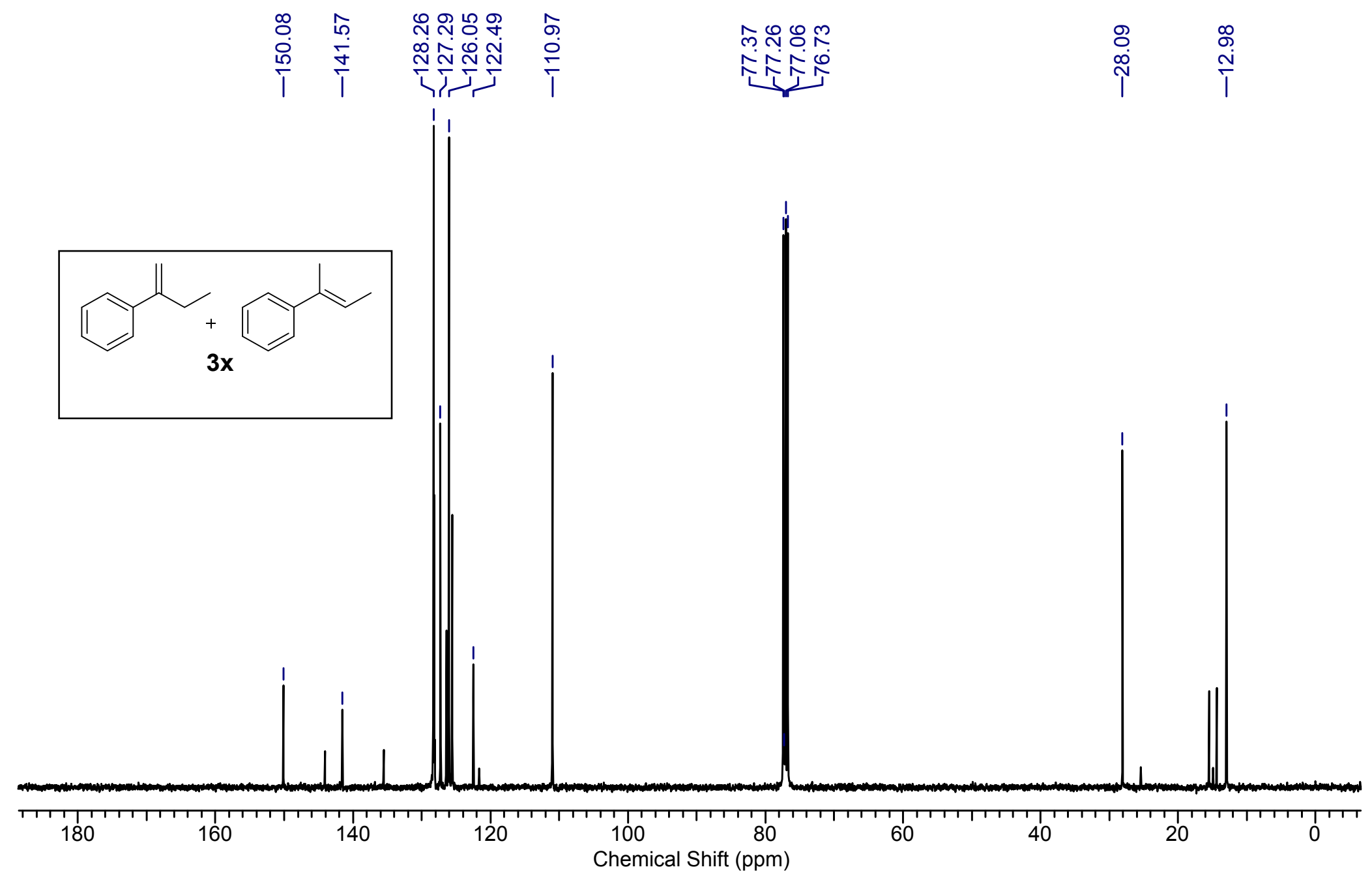

${ }^{13} \mathrm{C}\left\{{ }^{1} \mathrm{H}\right\}$ NMR of $3 \mathbf{x}\left(101 \mathrm{MHz}, \mathrm{CDCl}_{3}\right)$ 


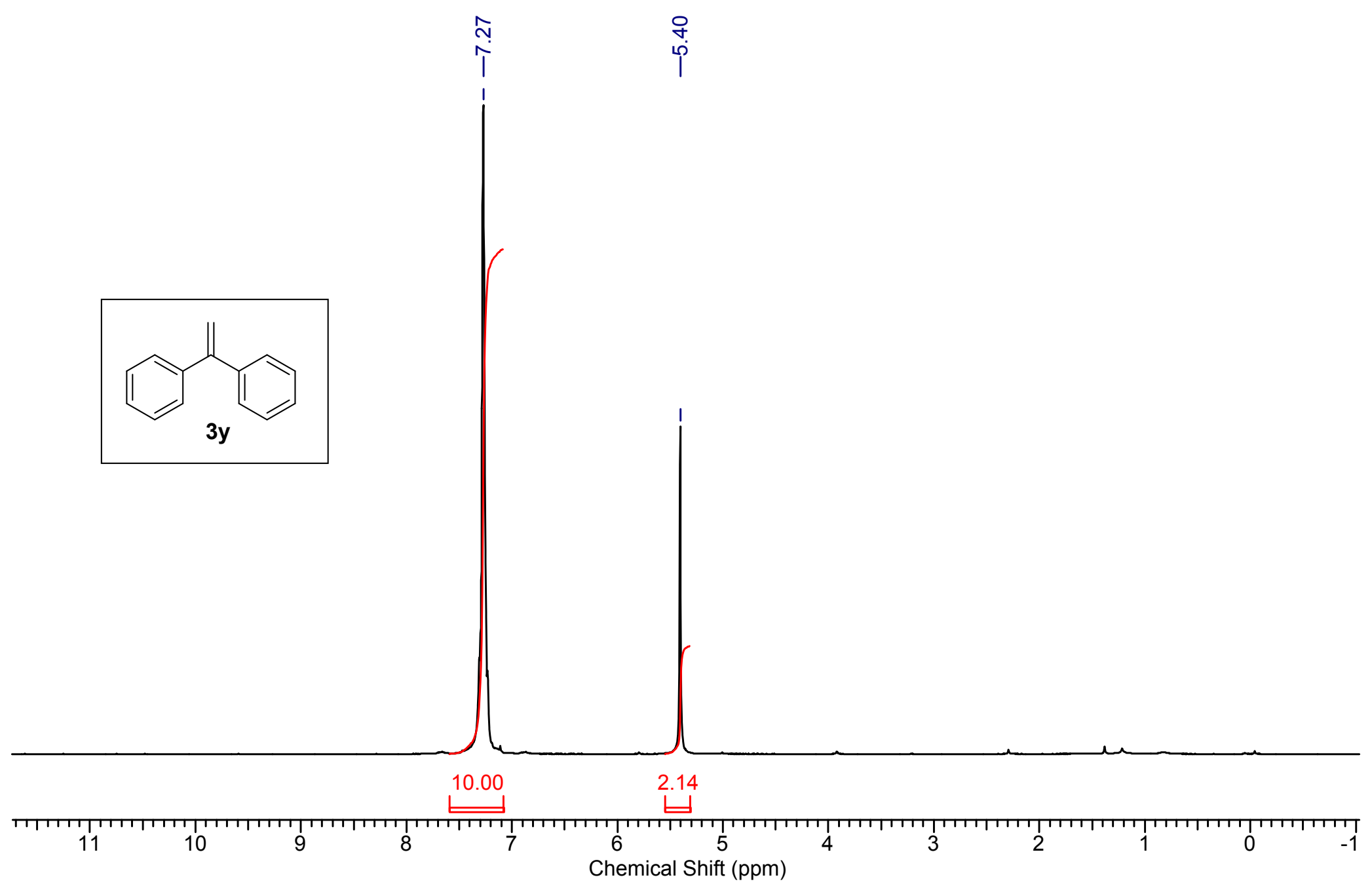

${ }^{1} \mathrm{H}$ NMR of $\mathbf{3 y}\left(200 \mathrm{MHz}, \mathrm{CDCl}_{3}\right)$ 


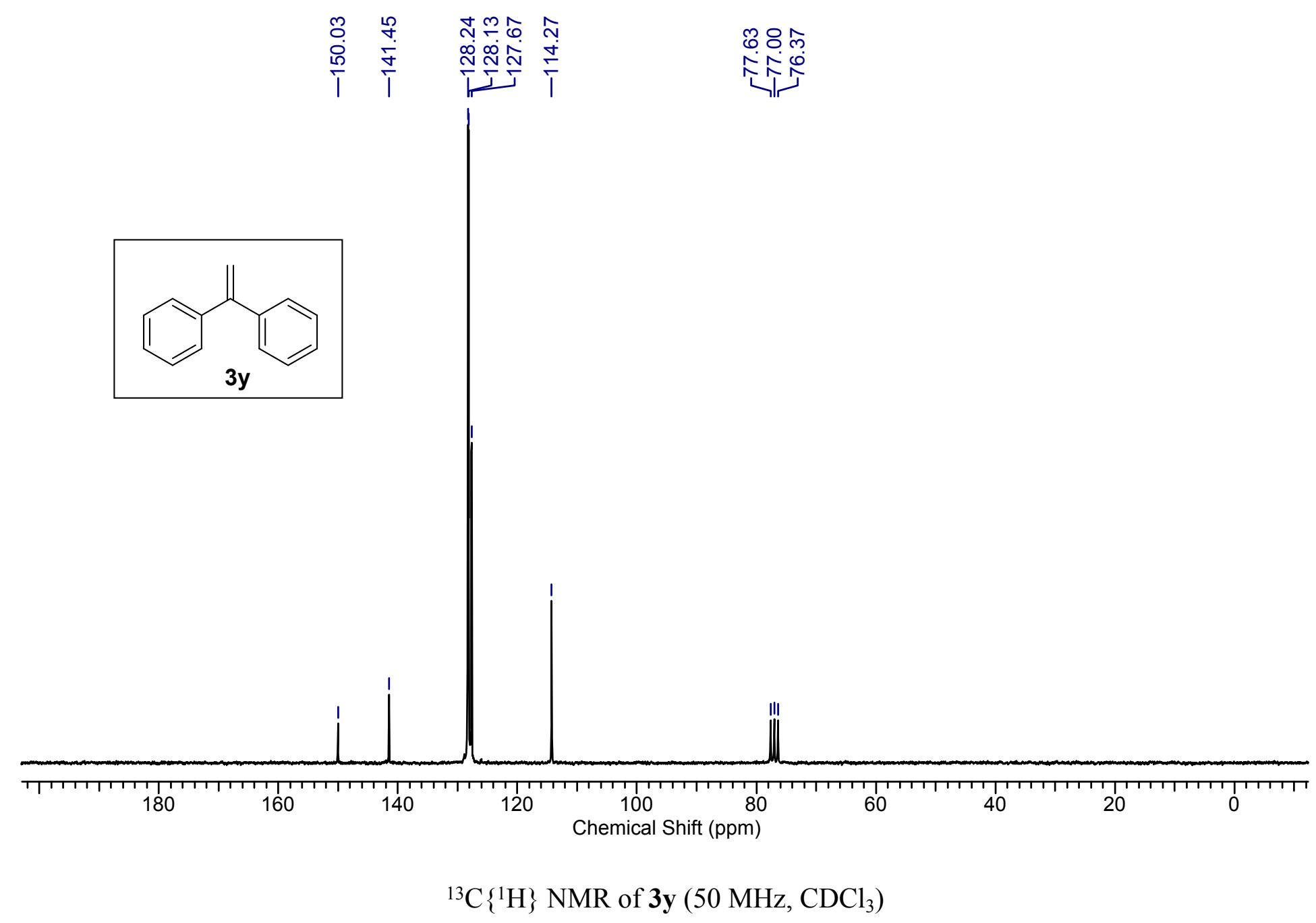




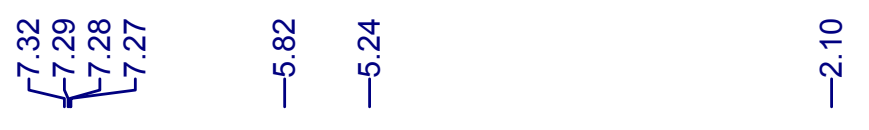
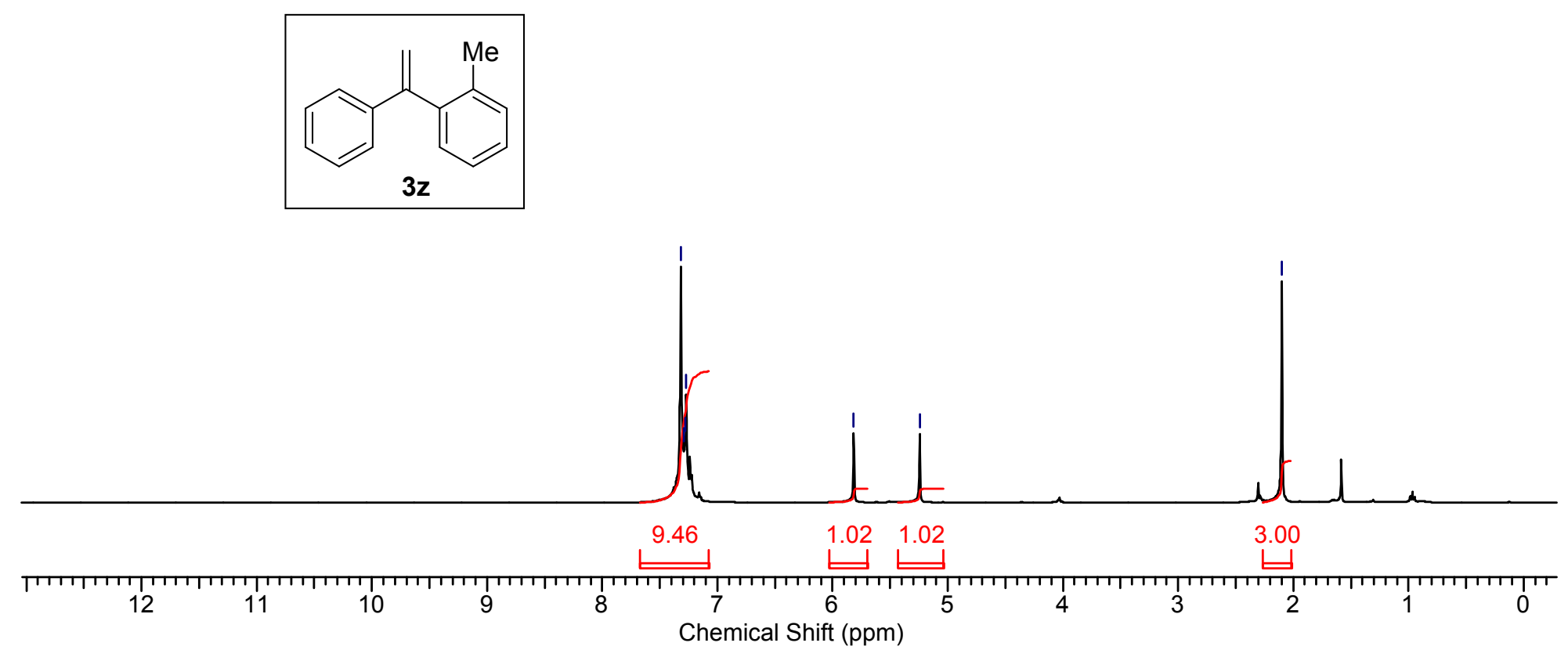

${ }^{1} \mathrm{H}$ NMR of $\mathbf{3 z}\left(400 \mathrm{MHz}, \mathrm{CDCl}_{3}\right)$ 


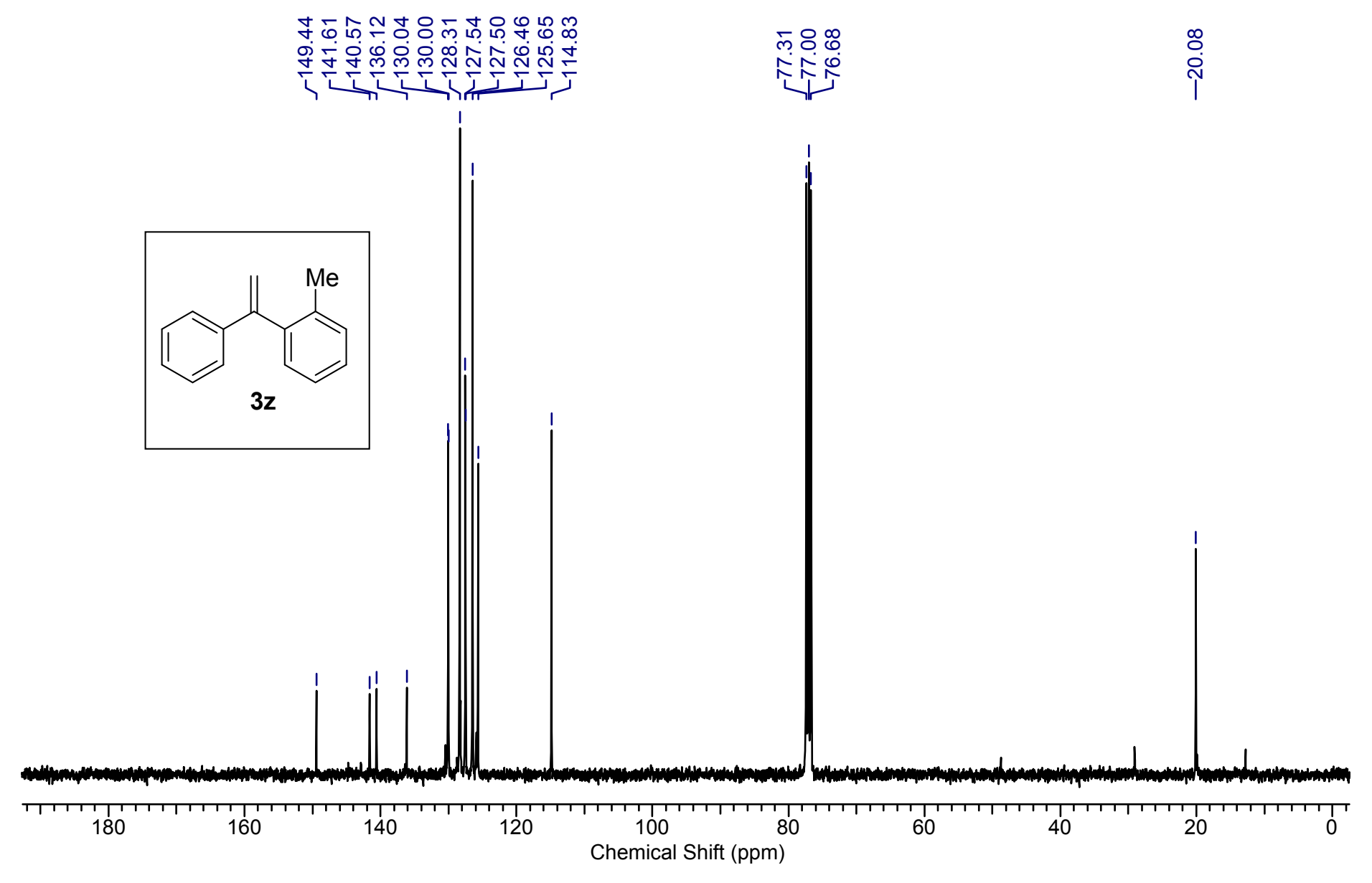

${ }^{13} \mathrm{C}\left\{{ }^{1} \mathrm{H}\right\}$ NMR of $\mathbf{3 z}\left(101 \mathrm{MHz}, \mathrm{CDCl}_{3}\right)$ 


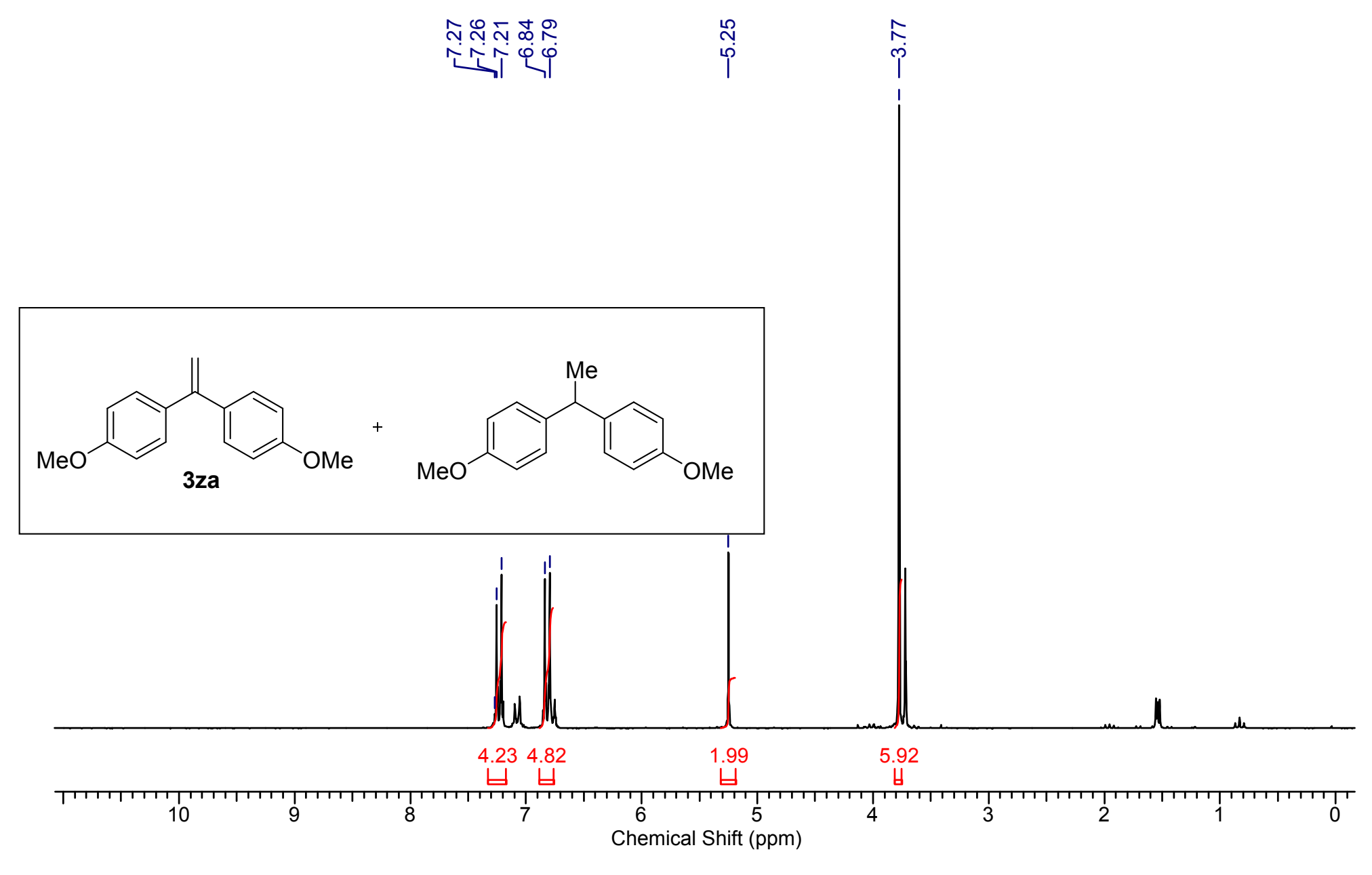

${ }^{1} \mathrm{H}$ NMR of 3za (200 MHz, $\mathrm{CDCl}_{3}$ ) 


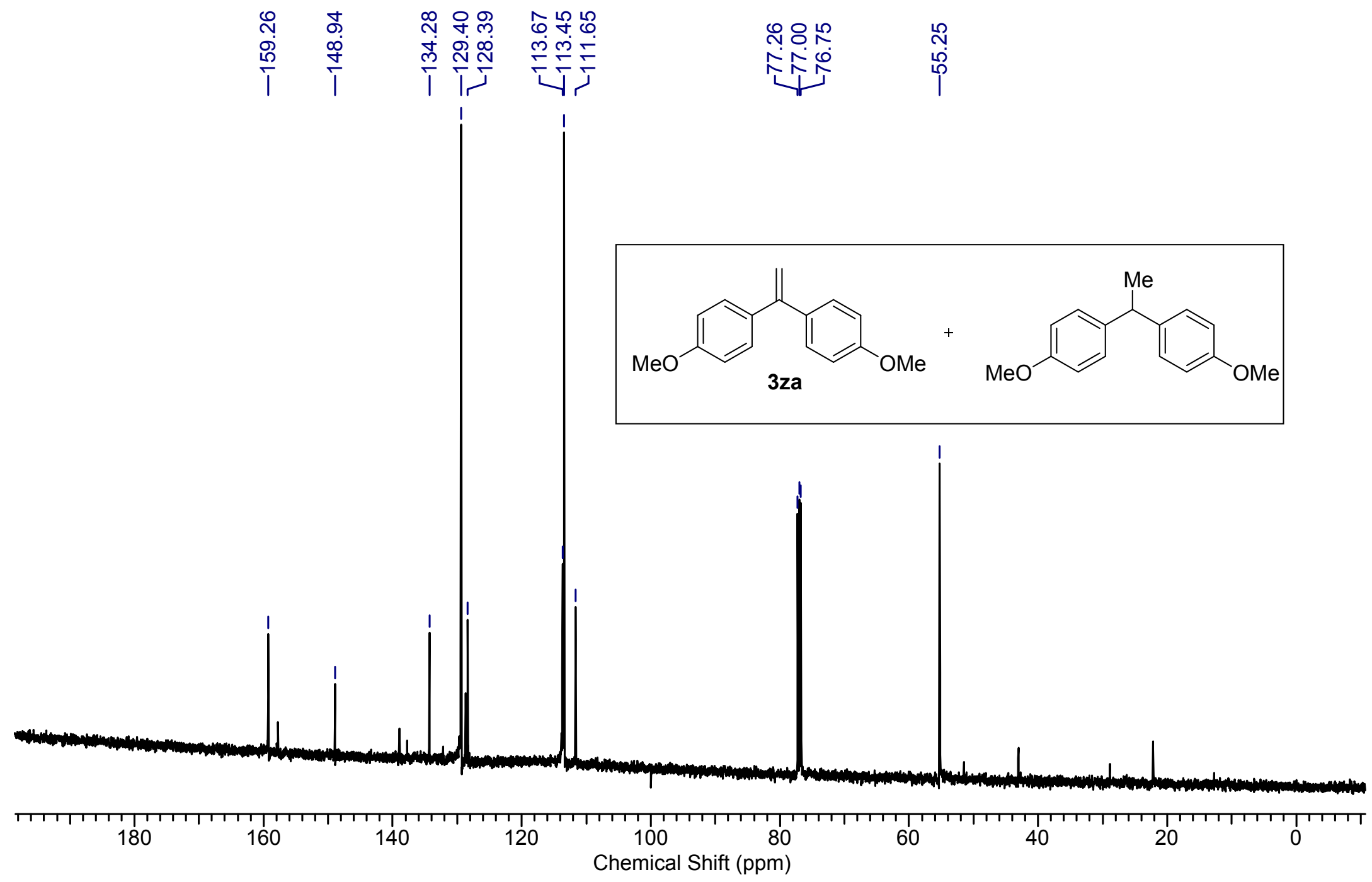

${ }^{13} \mathrm{C}\left\{{ }^{1} \mathrm{H}\right\}$ NMR of $3 z a\left(50 \mathrm{MHz}, \mathrm{CDCl}_{3}\right)$ 

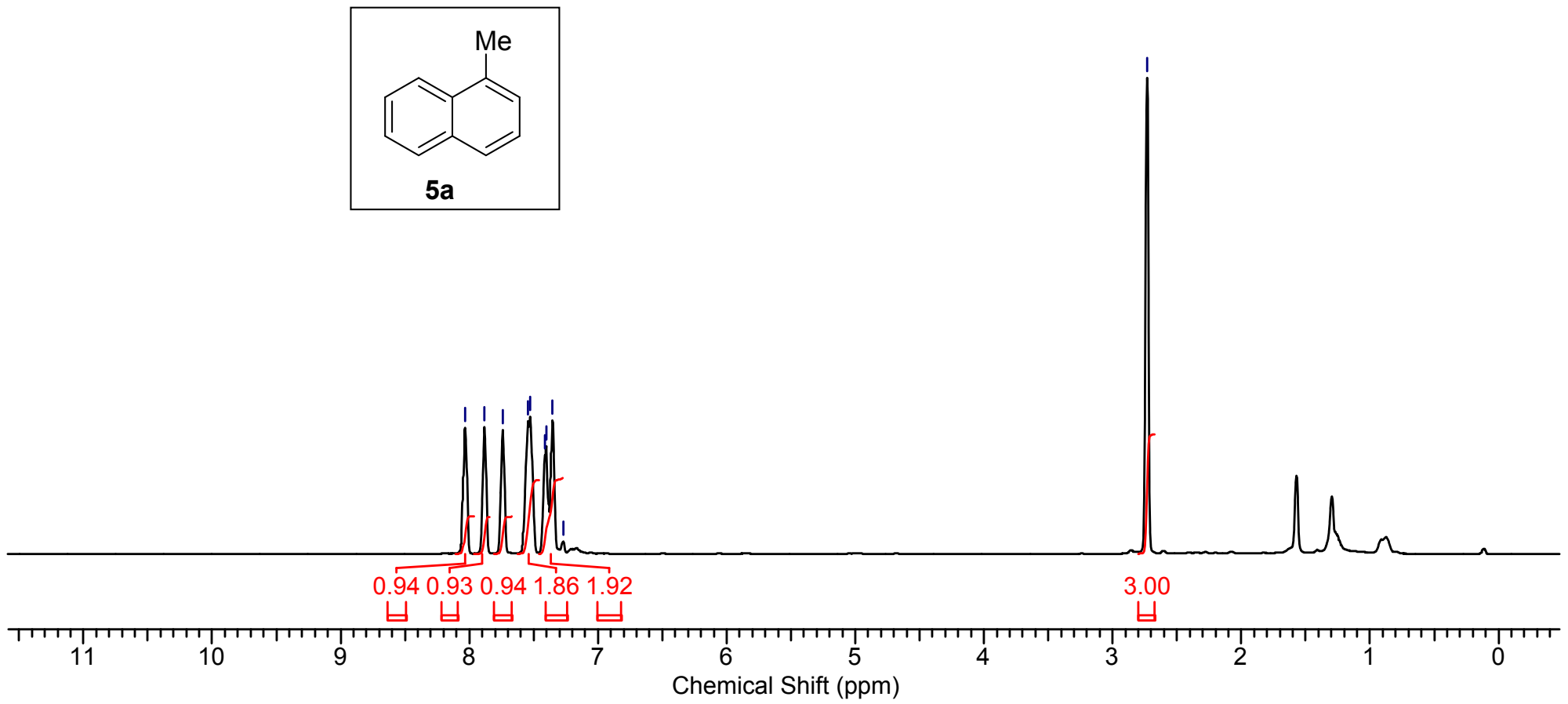

${ }^{1} \mathrm{H}$ NMR of $\mathbf{5 a}\left(500 \mathrm{MHz}, \mathrm{CDCl}_{3}\right)$ 


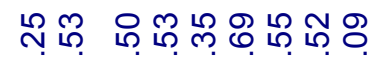

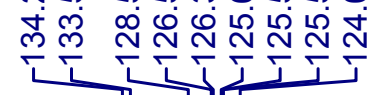

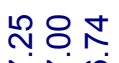

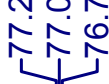

0
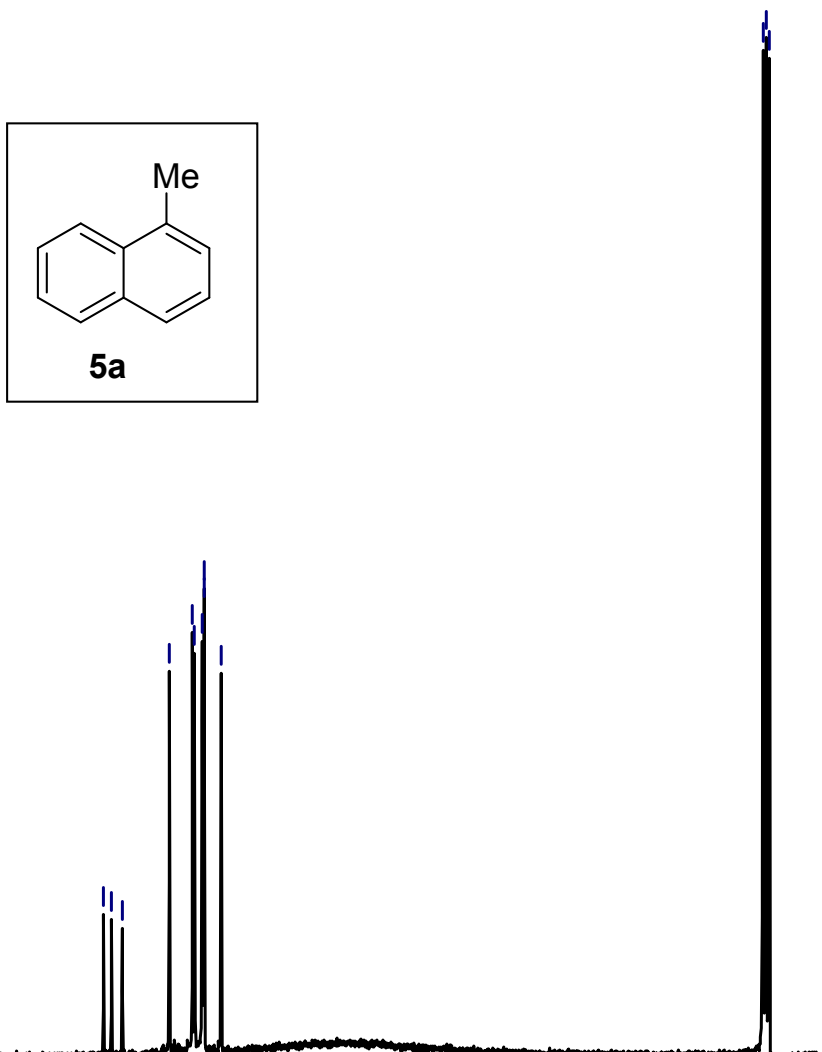

I

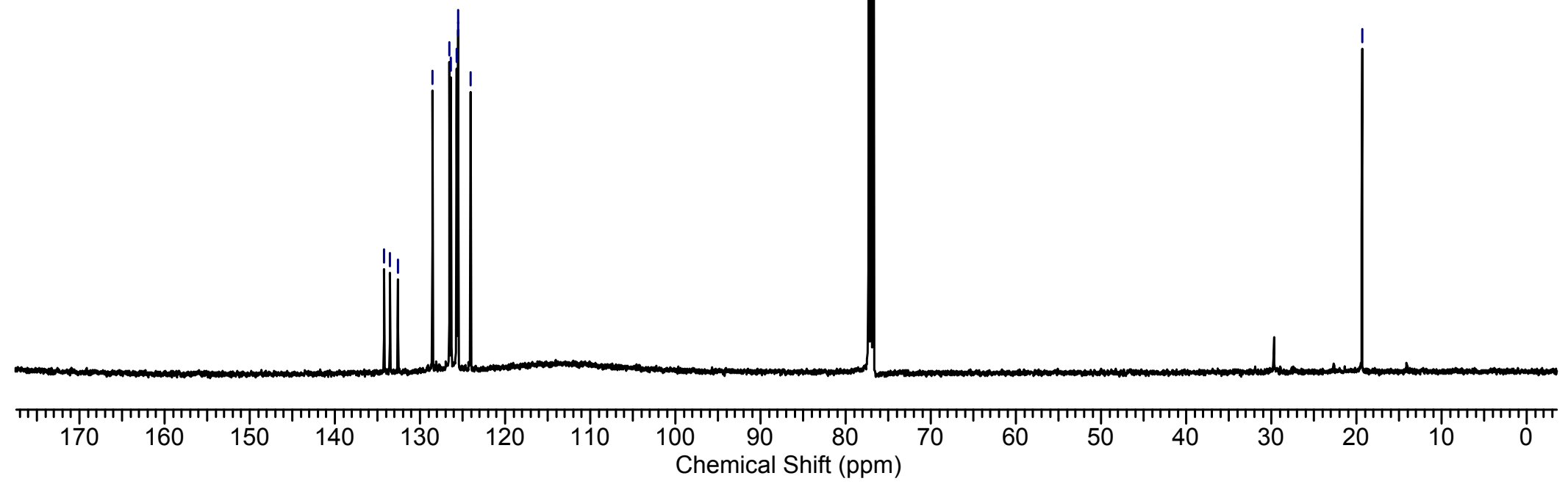

${ }^{13} \mathrm{C}\left\{{ }^{1} \mathrm{H}\right\} \mathrm{NMR}$ of $5 \mathbf{a}\left(126 \mathrm{MHz}, \mathrm{CDCl}_{3}\right)$ 
సัก

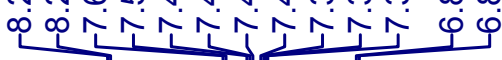

\section{ஜำธธธธอ8} ن.
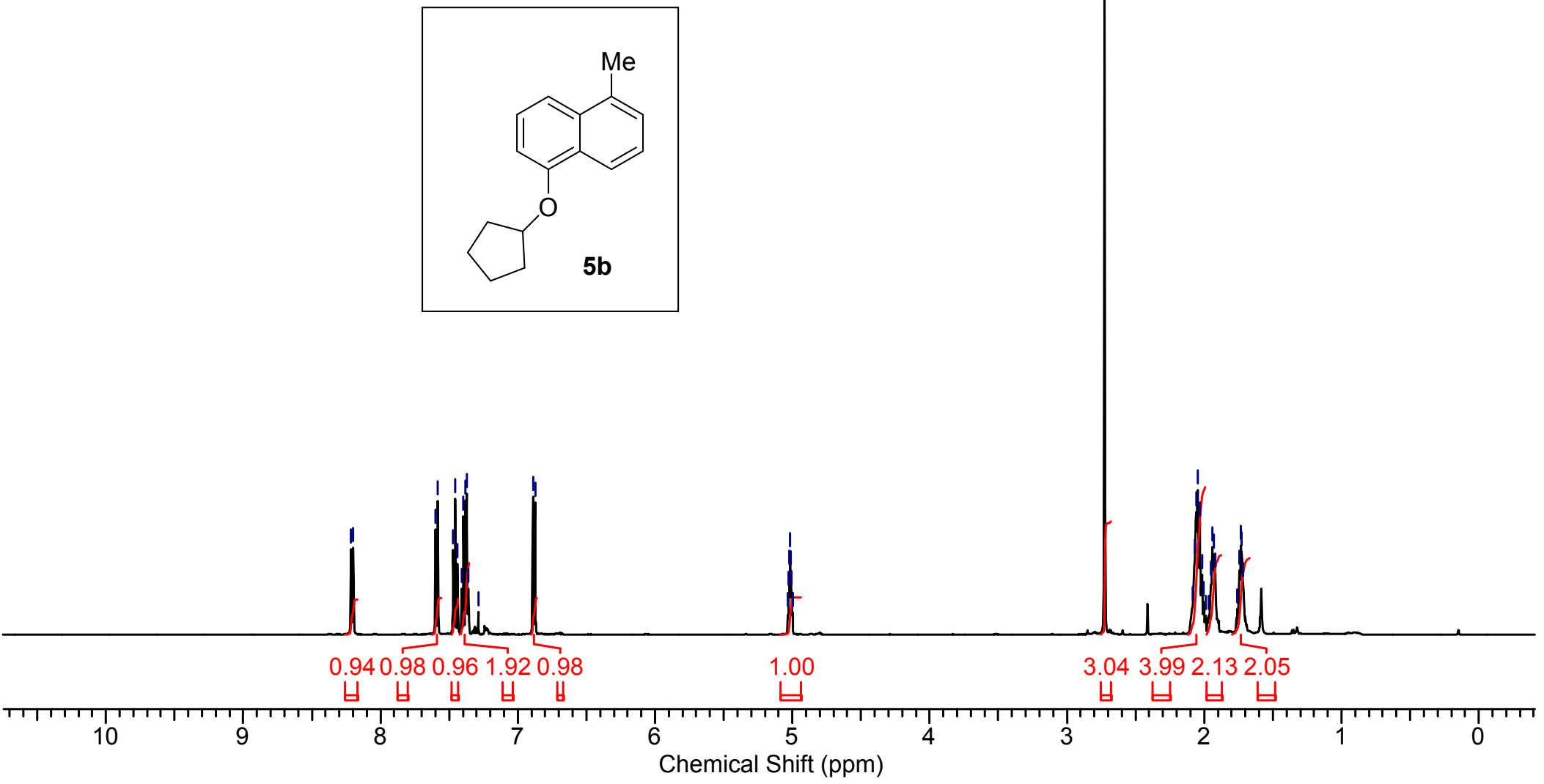

${ }^{1} \mathrm{H}$ NMR of $\mathbf{5 b}\left(500 \mathrm{MHz}, \mathrm{CDCl}_{3}\right)$ 


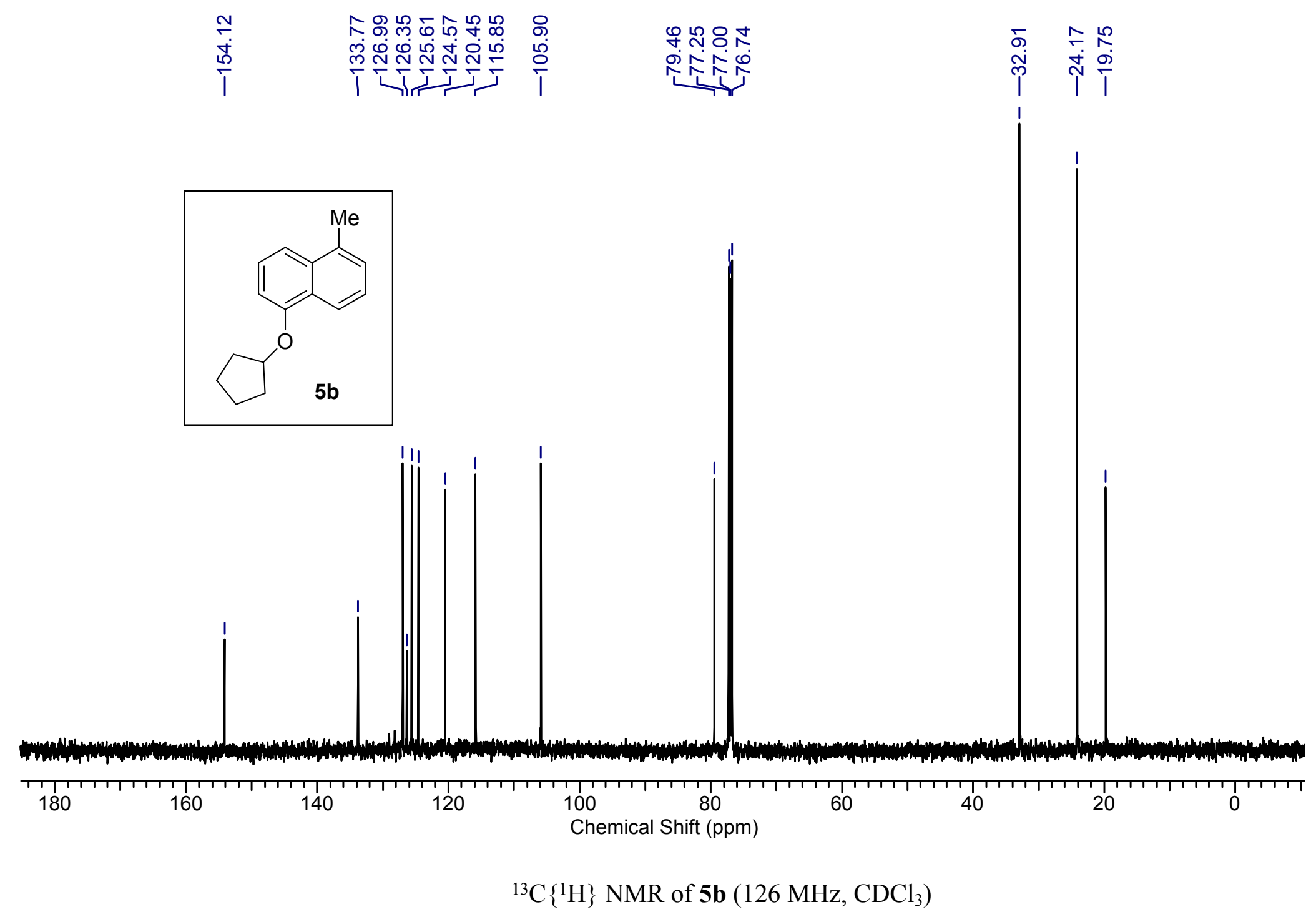




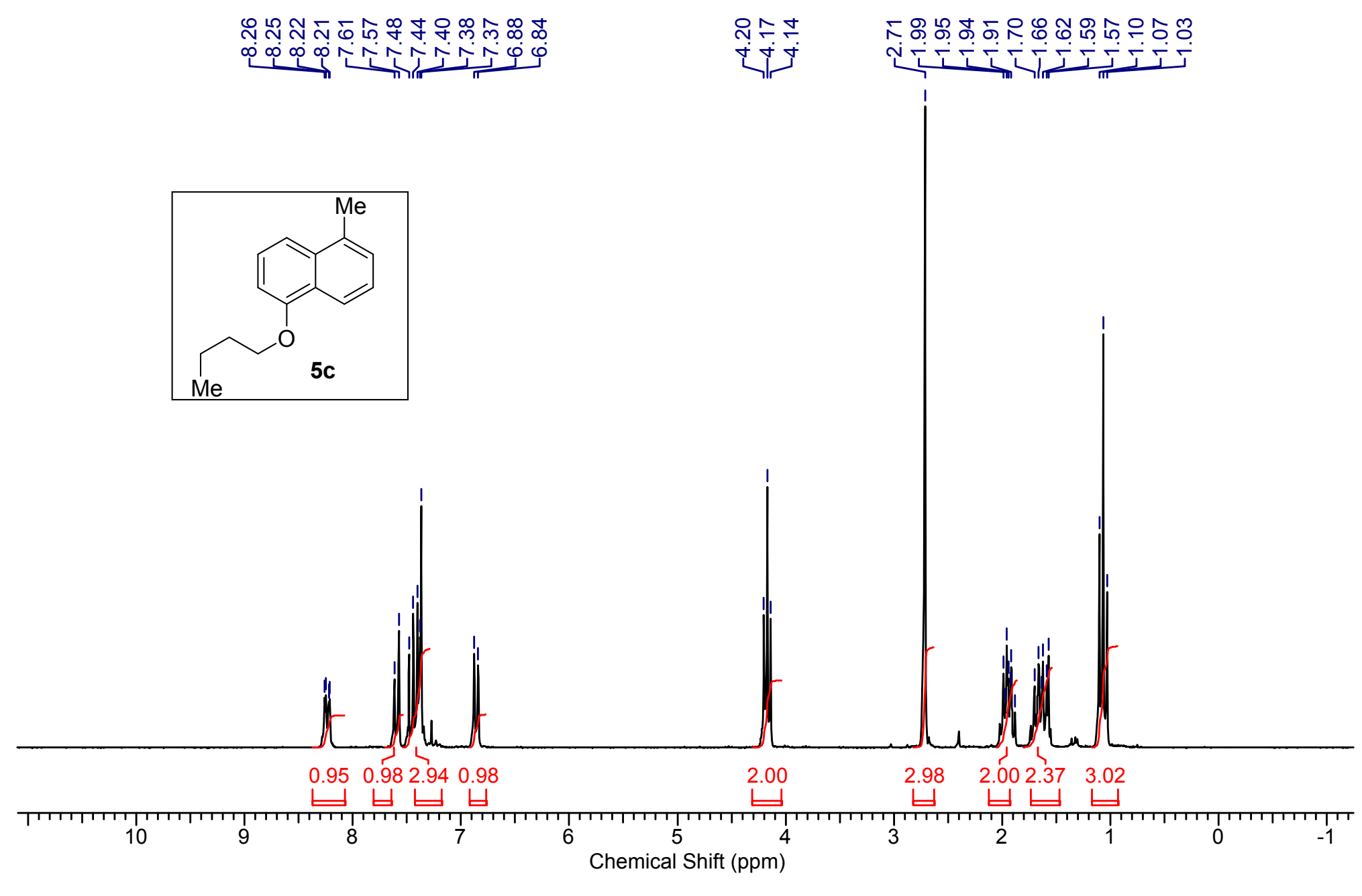

${ }^{1} \mathrm{H}$ NMR of $5 \mathbf{c}\left(200 \mathrm{MHz}, \mathrm{CDCl}_{3}\right)$ 


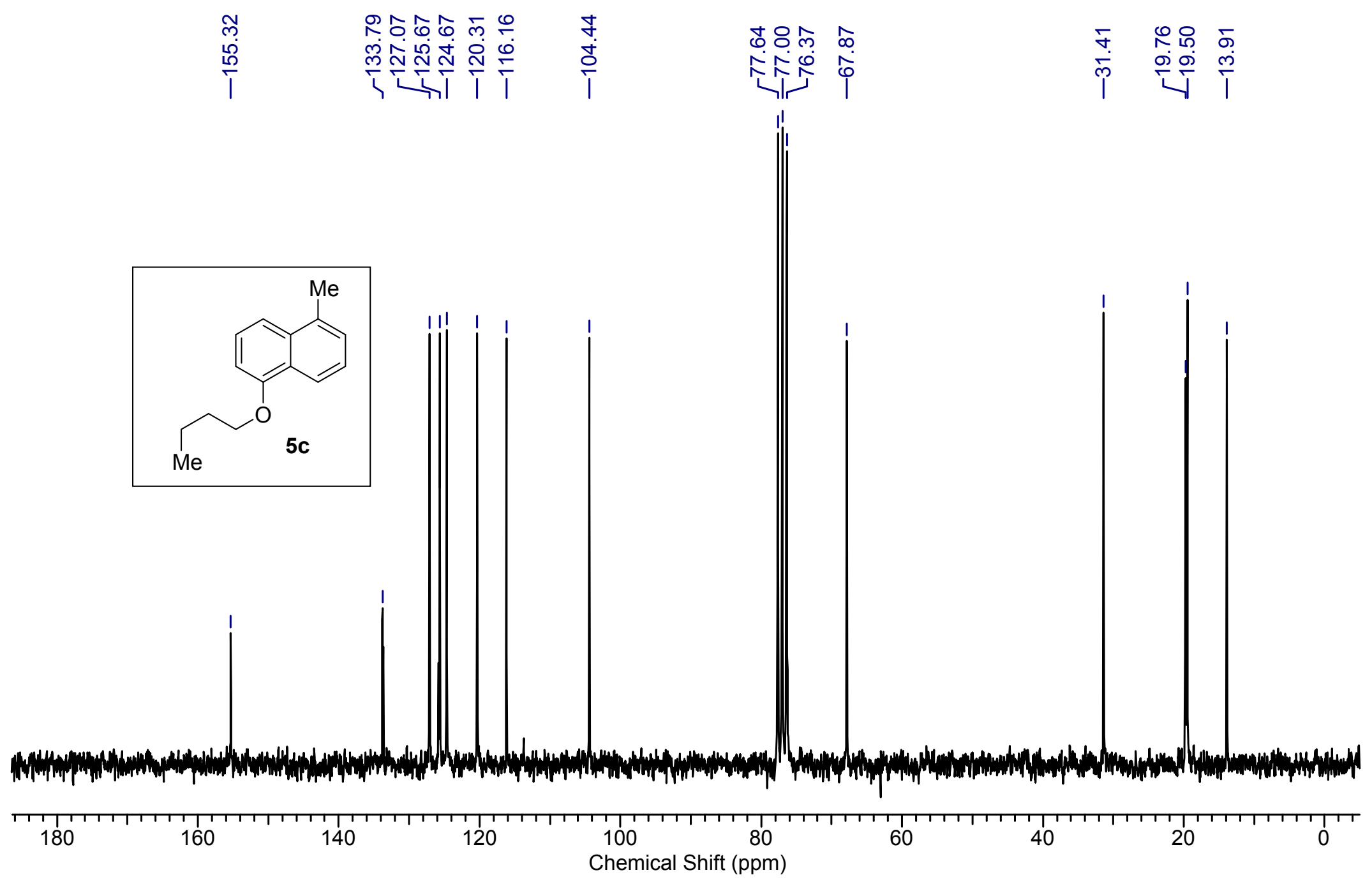

${ }^{13} \mathrm{C}\left\{{ }^{1} \mathrm{H}\right\}$ NMR of $5 \mathbf{c}\left(50 \mathrm{MHz}, \mathrm{CDCl}_{3}\right)$ 


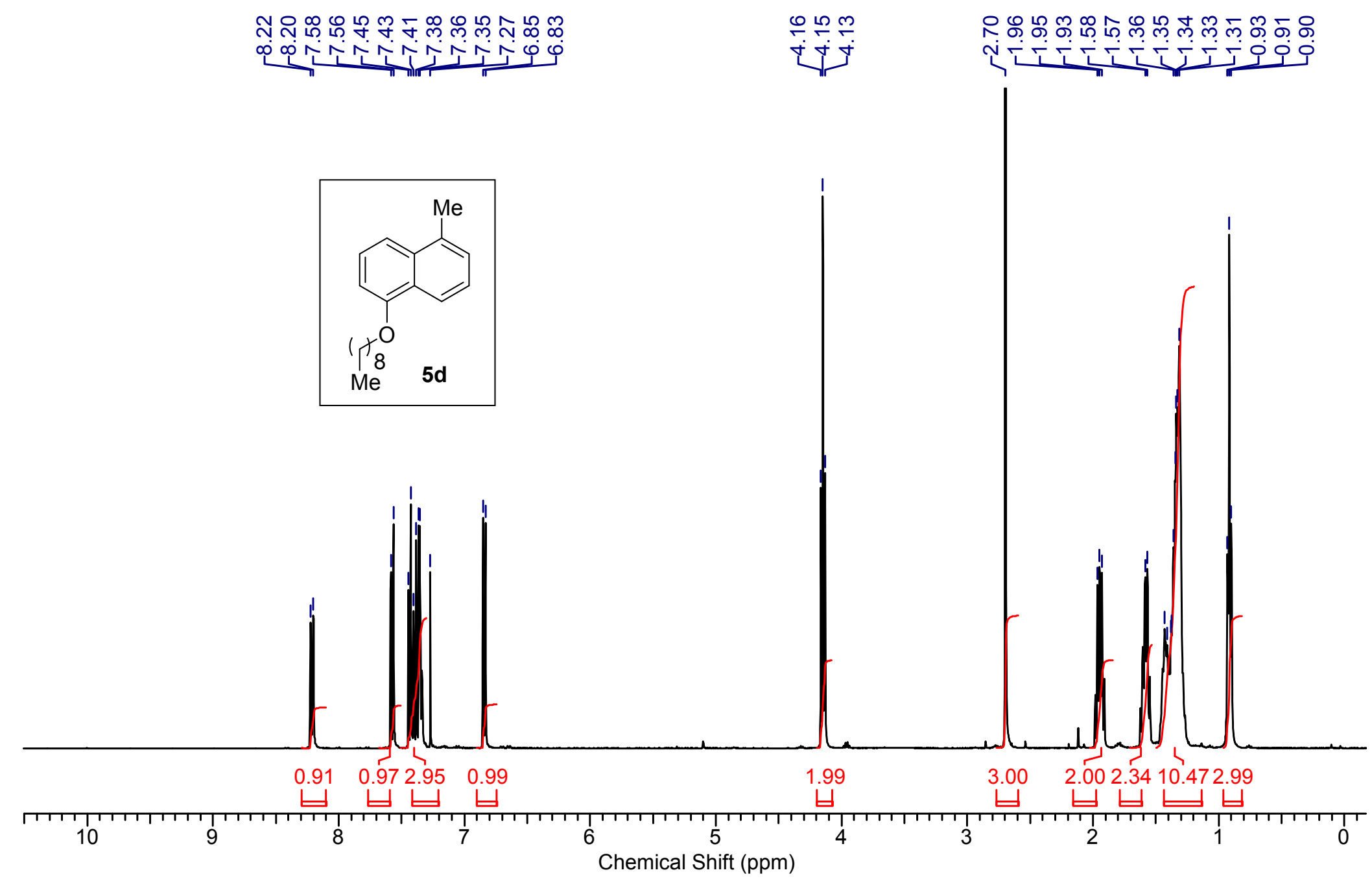

${ }^{1} \mathrm{H}$ NMR of $5 \mathbf{d}\left(400 \mathrm{MHz}, \mathrm{CDCl}_{3}\right)$ 


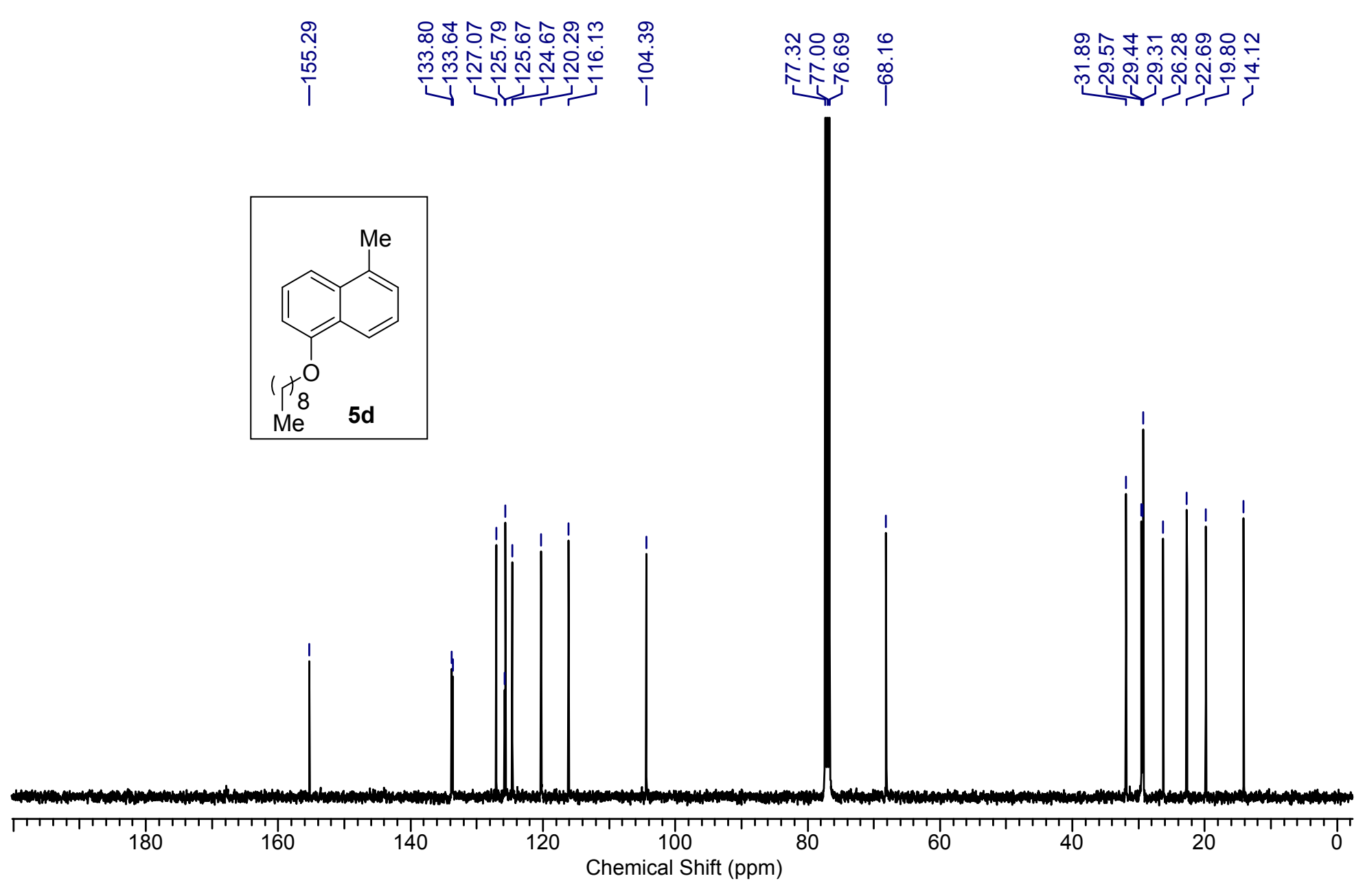

${ }^{13} \mathrm{C}\left\{{ }^{1} \mathrm{H}\right\} \mathrm{NMR}$ of $\mathbf{5 d}\left(101 \mathrm{MHz}, \mathrm{CDCl}_{3}\right)$ 


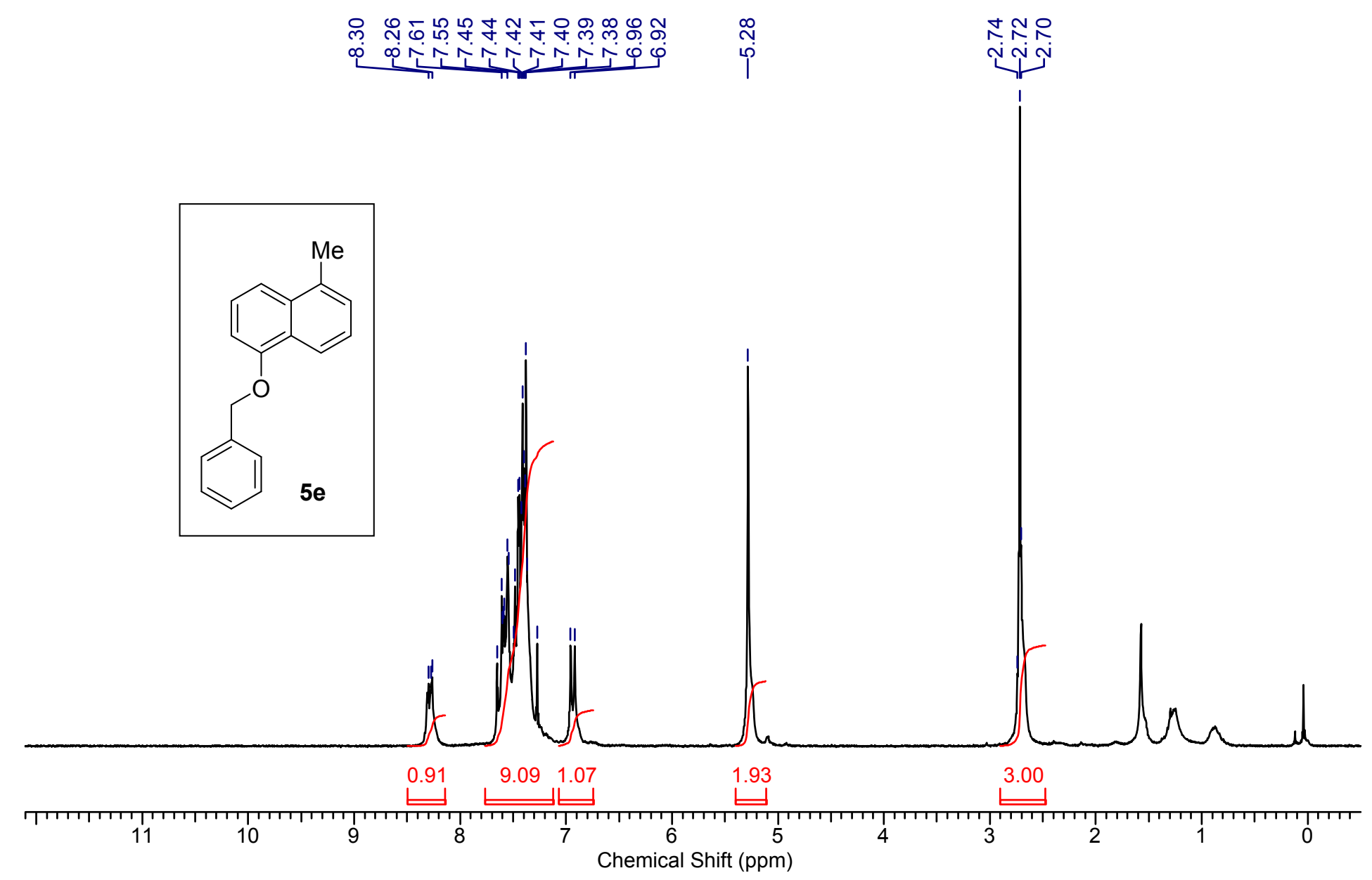

${ }^{1} \mathrm{H}$ NMR of $5 \mathbf{d}\left(200 \mathrm{MHz}, \mathrm{CDCl}_{3}\right)$ 

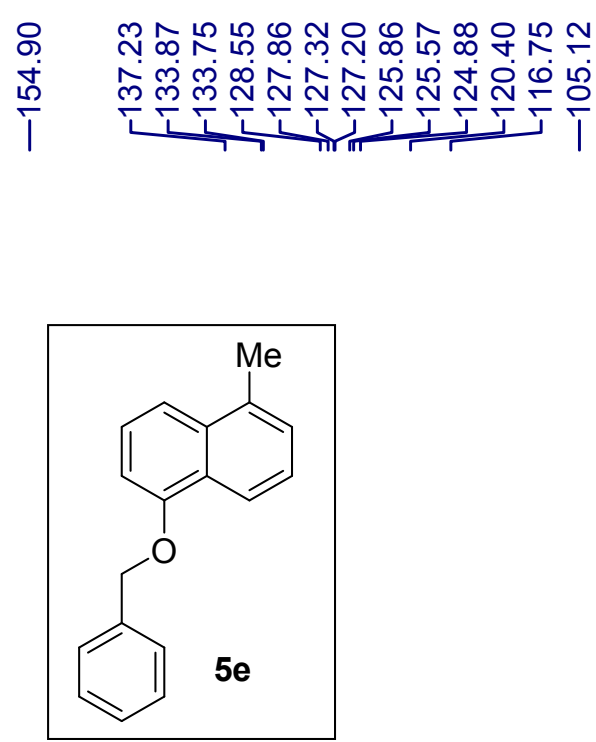

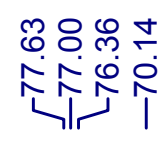

$\stackrel{0}{N}$
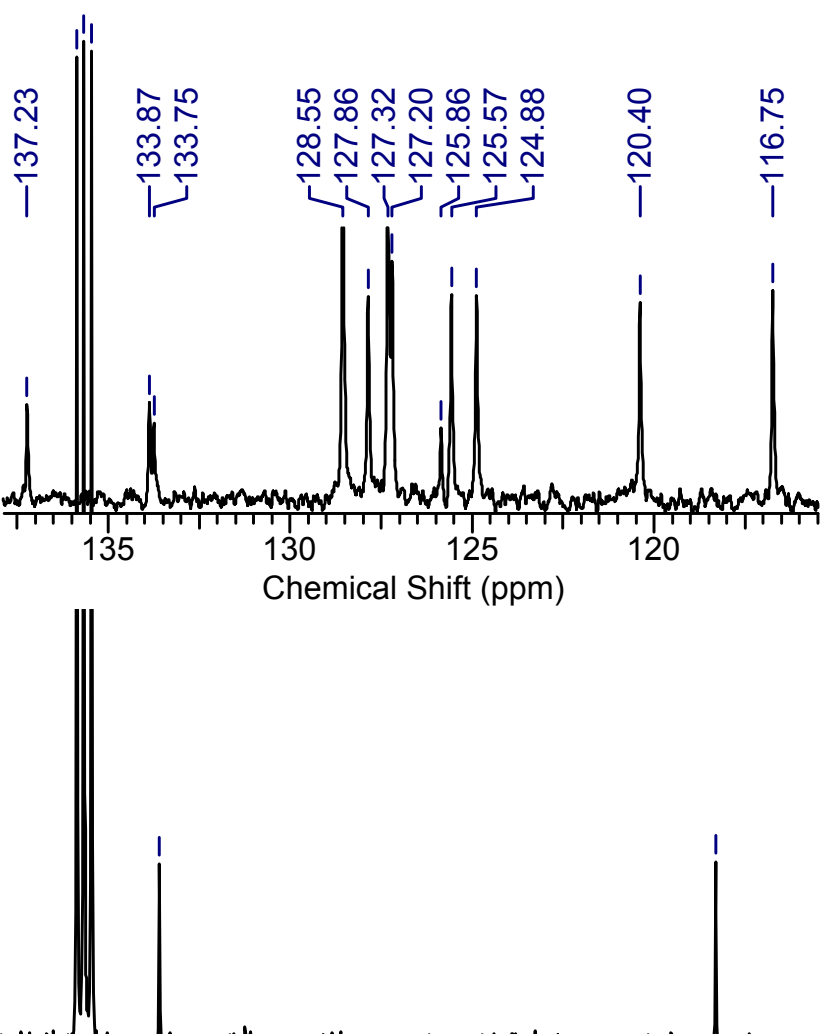

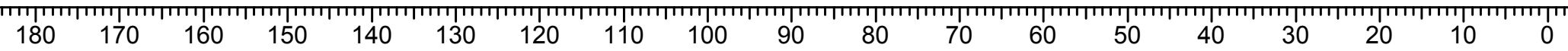
Chemical Shift (ppm)

${ }^{13} \mathrm{C}\left\{{ }^{1} \mathrm{H}\right\} \mathrm{NMR}$ of $5 \mathbf{e}\left(50 \mathrm{MHz}, \mathrm{CDCl}_{3}\right)$ 


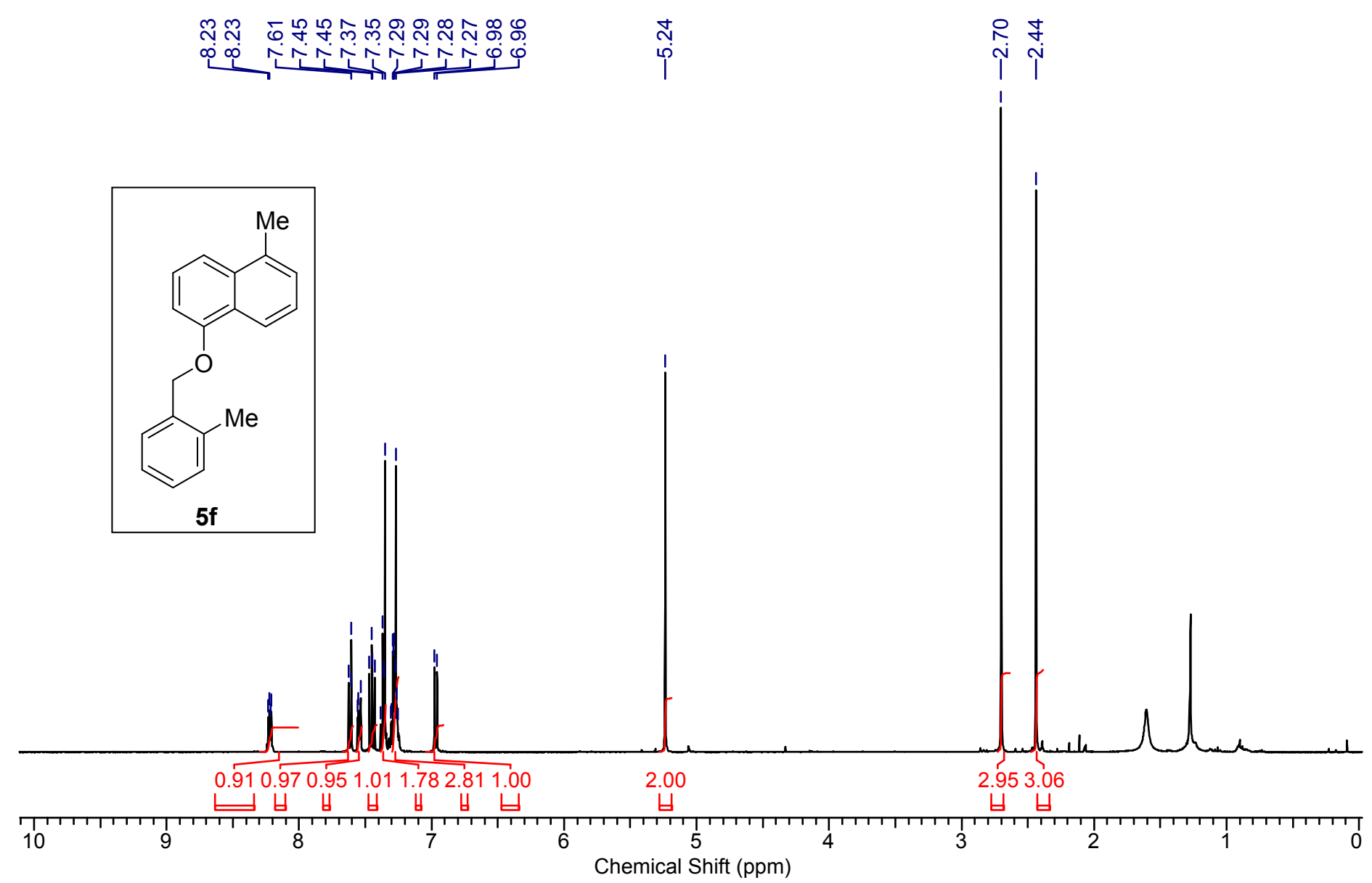

${ }^{1} \mathrm{H}$ NMR of $\mathbf{5 f}\left(400 \mathrm{MHz}, \mathrm{CDCl}_{3}\right)$ 


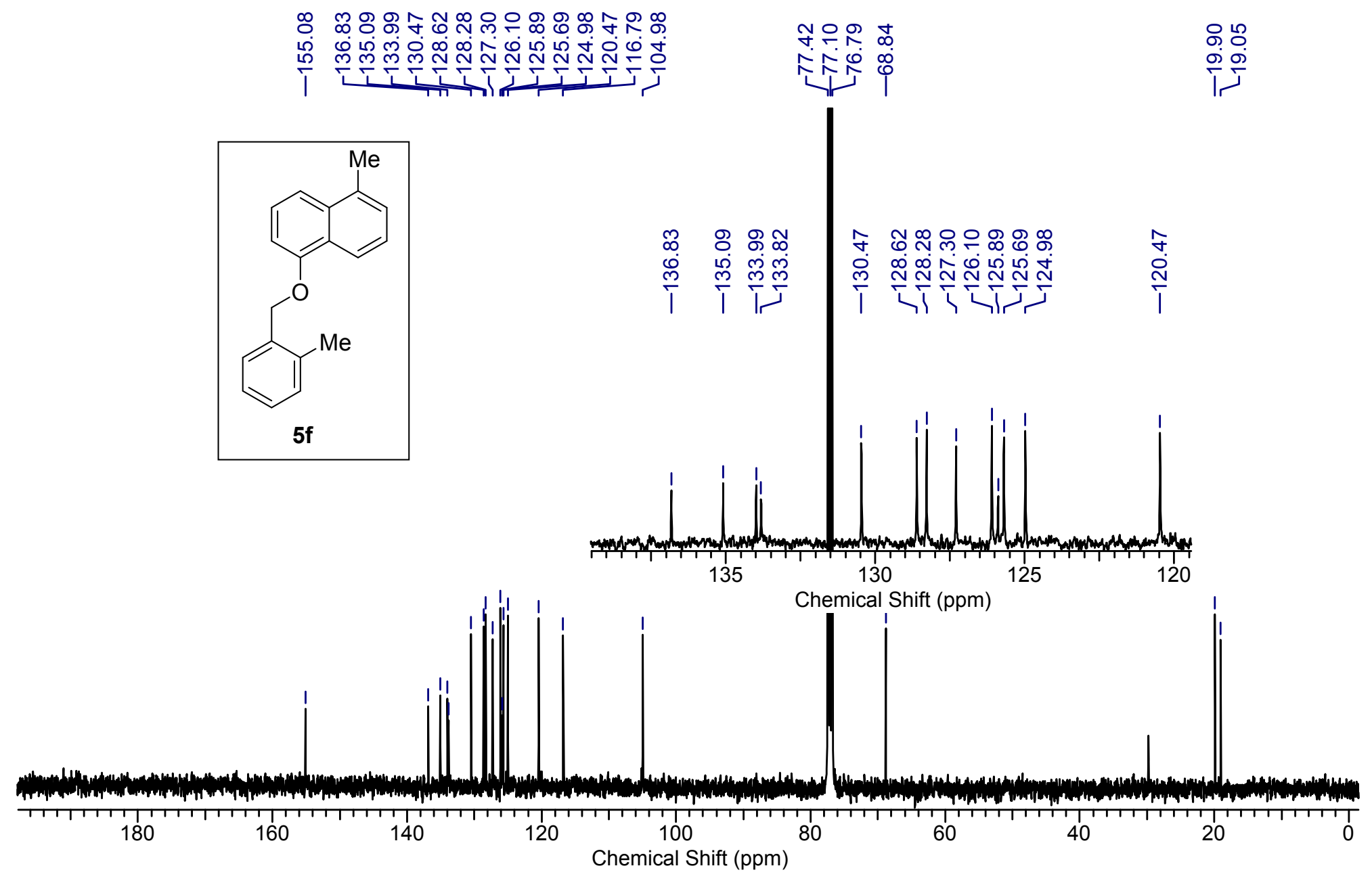

${ }^{13} \mathrm{C}\left\{{ }^{1} \mathrm{H}\right\} \mathrm{NMR}$ of $\mathbf{5 f}\left(101 \mathrm{MHz}, \mathrm{CDCl}_{3}\right)$ 


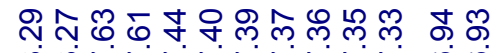

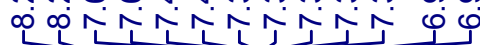
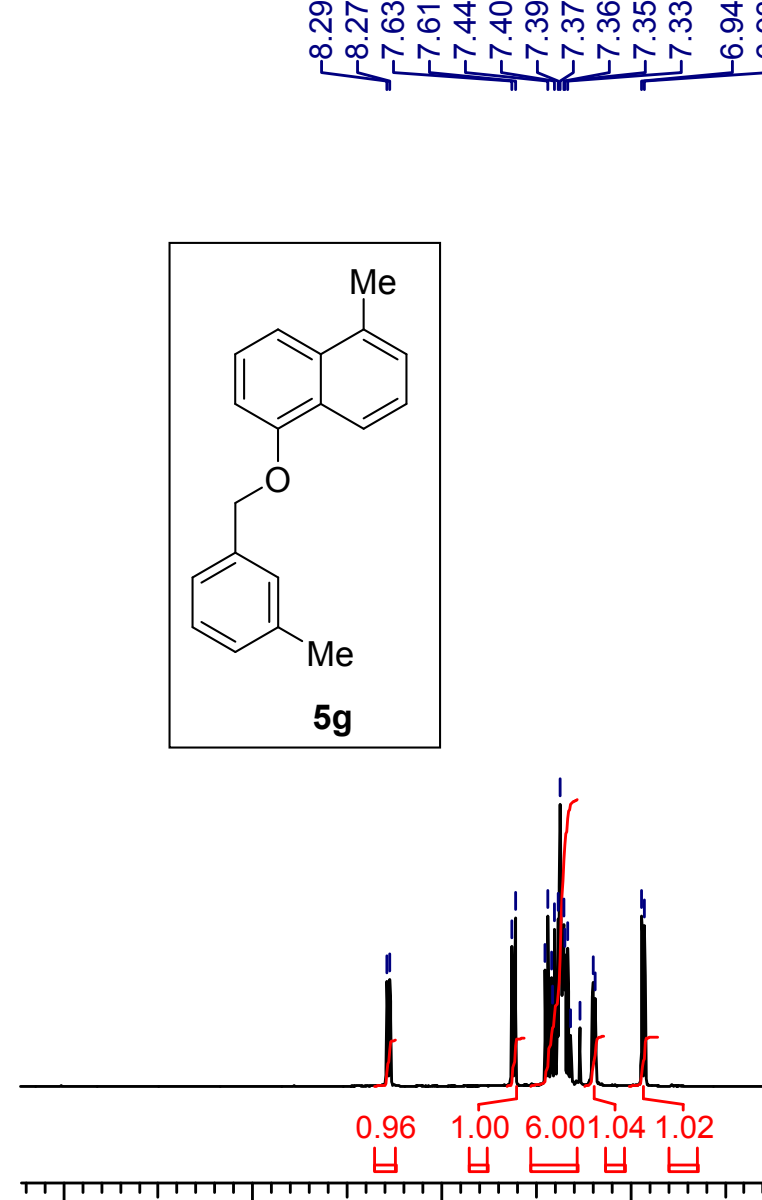

10

9

8

7

Chemical Shift (ppm)

${ }^{1} \mathrm{H}$ NMR of $\mathbf{5 g}\left(500 \mathrm{MHz}, \mathrm{CDCl}_{3}\right)$ 


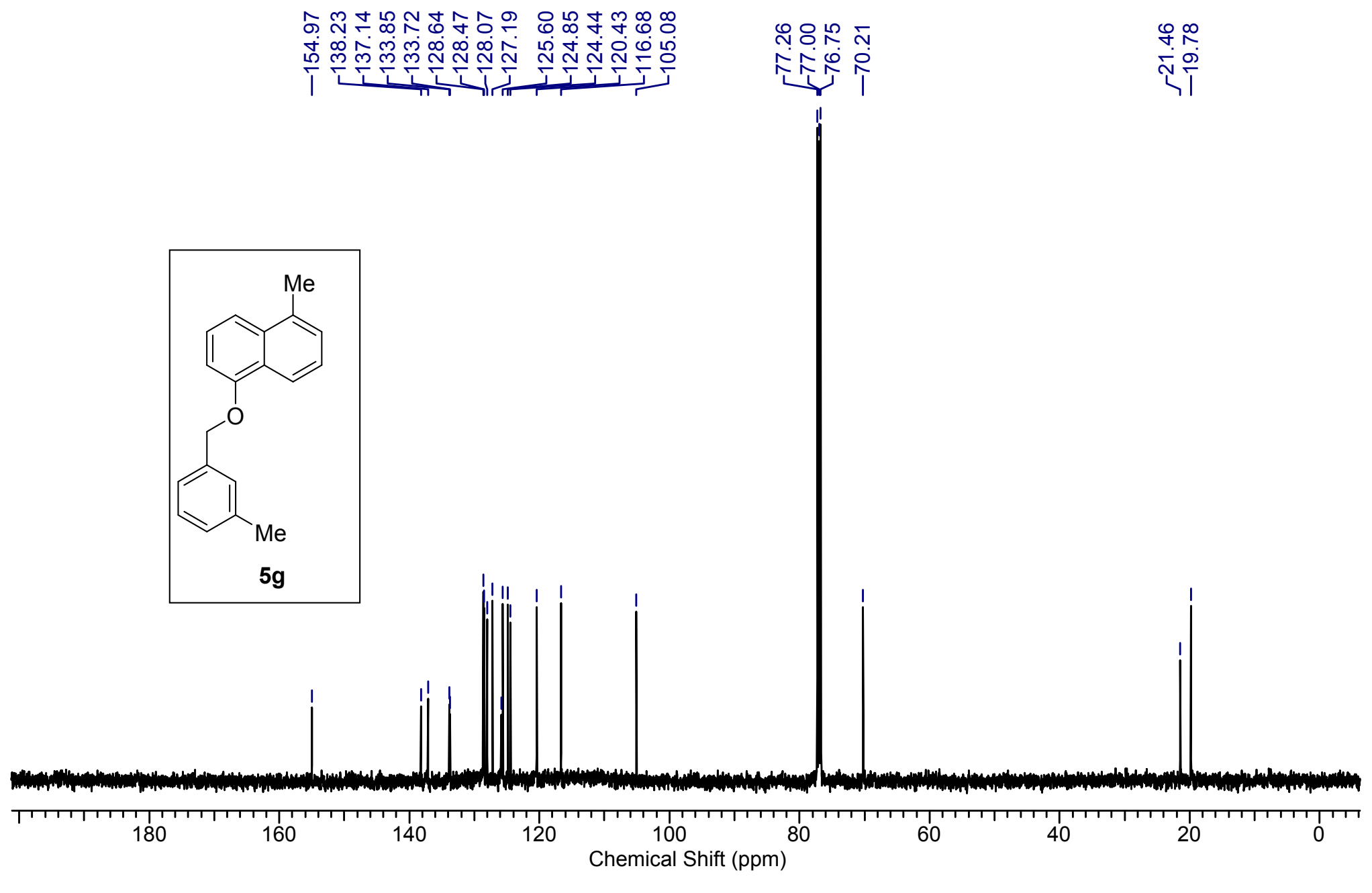

${ }^{13} \mathrm{C}\left\{{ }^{1} \mathrm{H}\right\} \mathrm{NMR}$ of $\mathbf{5 g}\left(126 \mathrm{MHz}, \mathrm{CDCl}_{3}\right)$ 


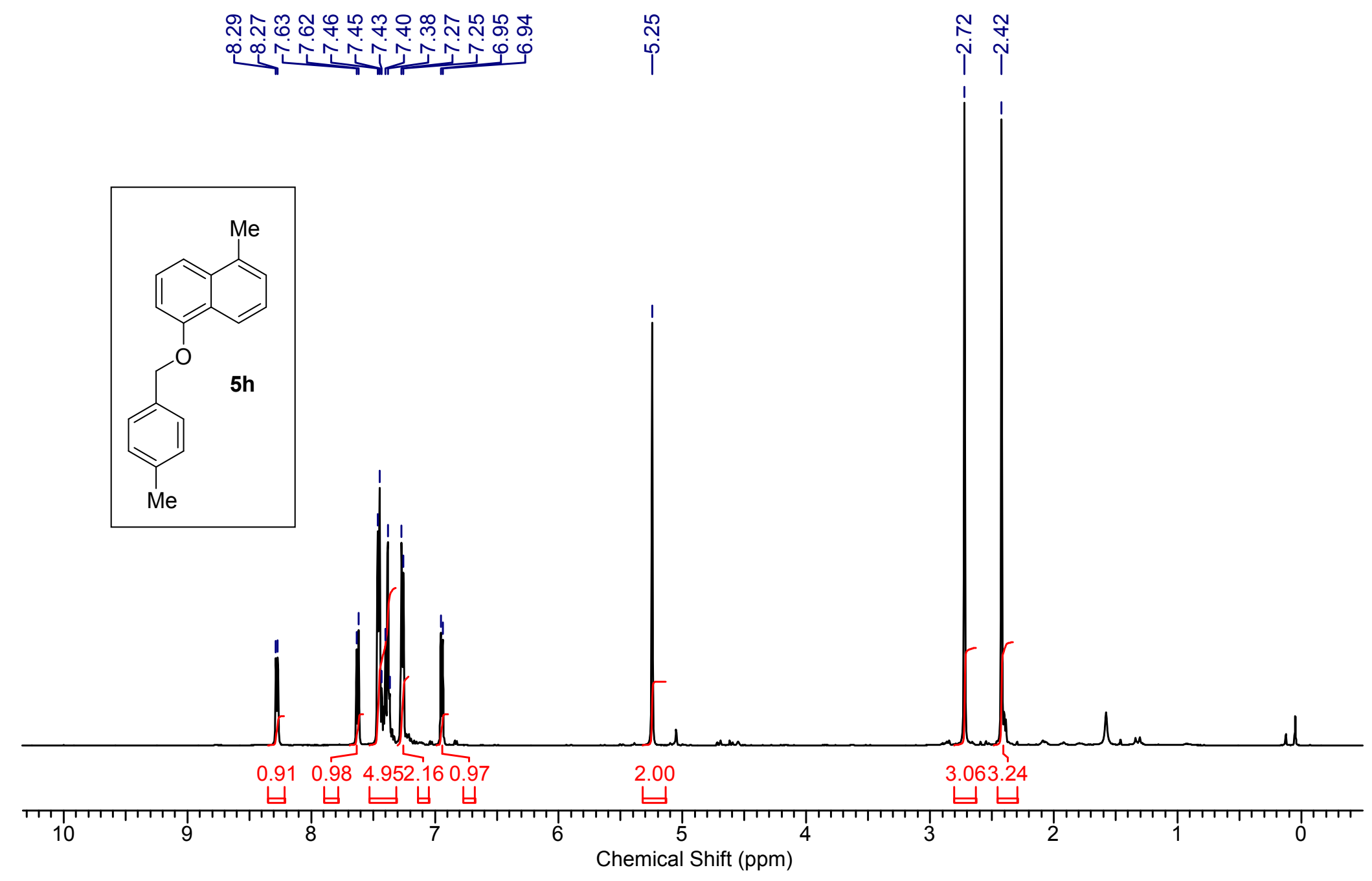

${ }^{1} \mathrm{H}$ NMR of $\mathbf{5 h}\left(500 \mathrm{MHz}, \mathrm{CDCl}_{3}\right)$ 


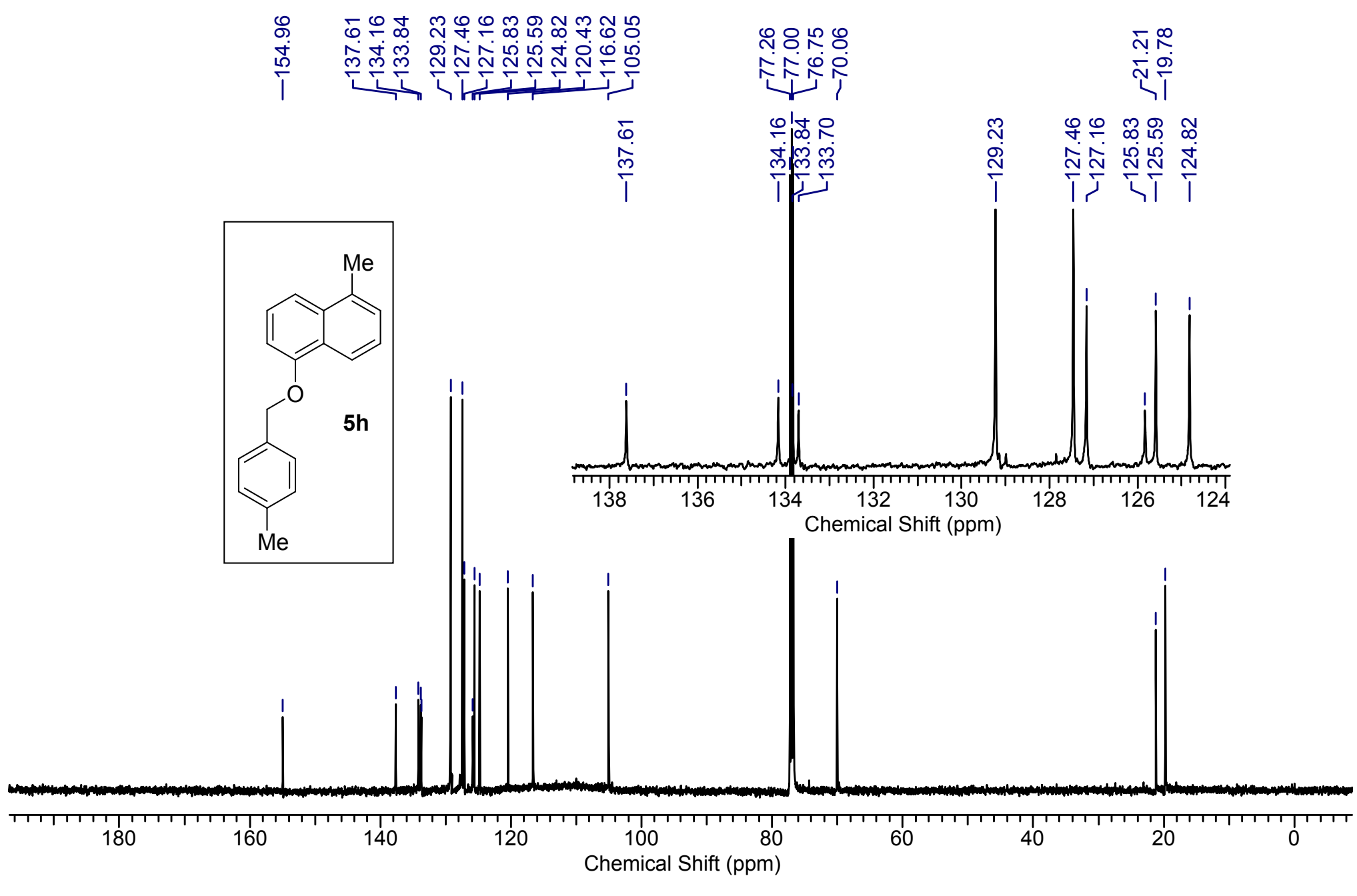

${ }^{13} \mathrm{C}\left\{{ }^{1} \mathrm{H}\right\} \mathrm{NMR}$ of $\mathbf{5 h}\left(126 \mathrm{MHz}, \mathrm{CDCl}_{3}\right)$ 


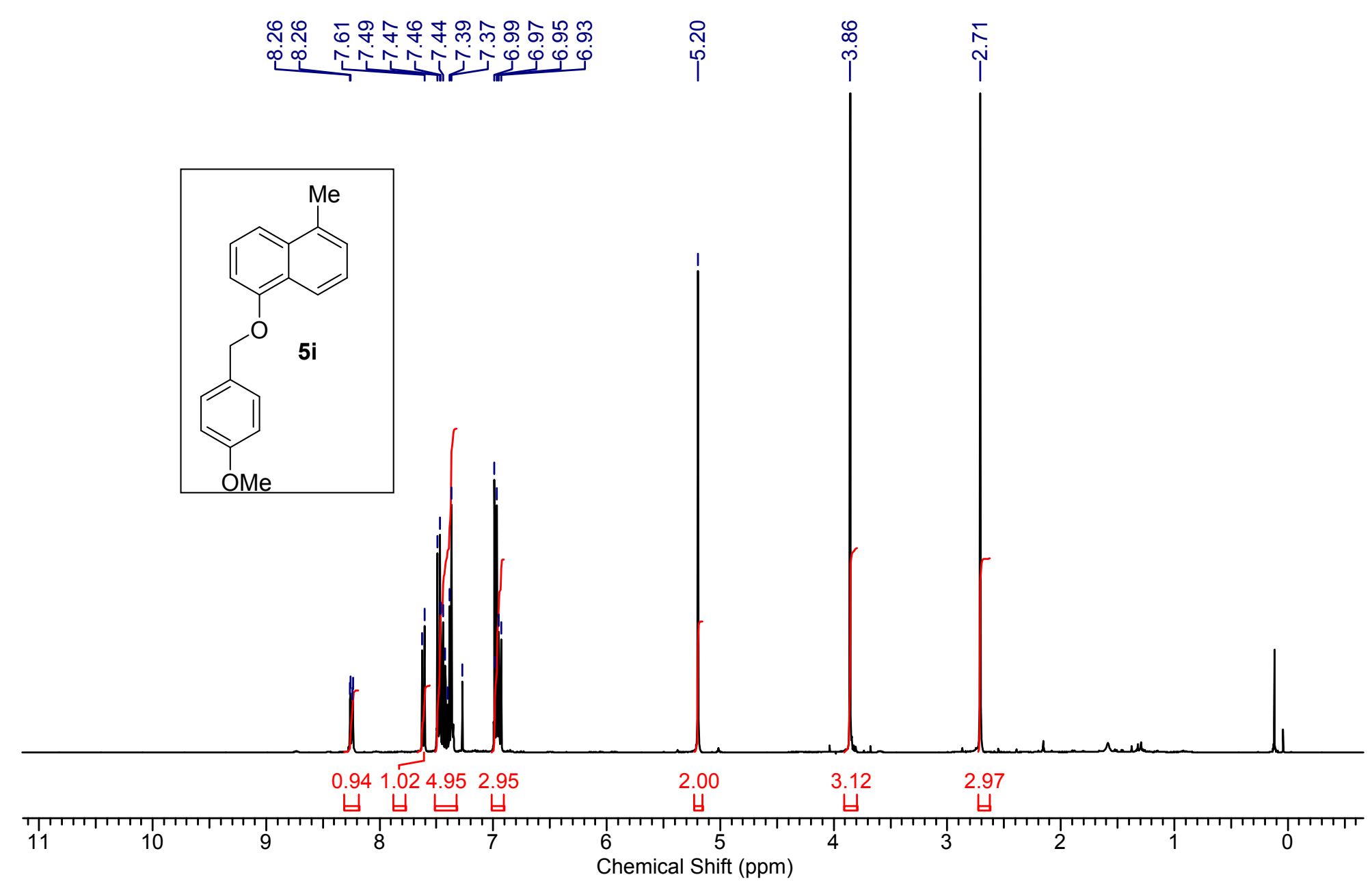

${ }^{1} \mathrm{H}$ NMR of $5 \mathbf{i}\left(400 \mathrm{MHz}, \mathrm{CDCl}_{3}\right)$ 


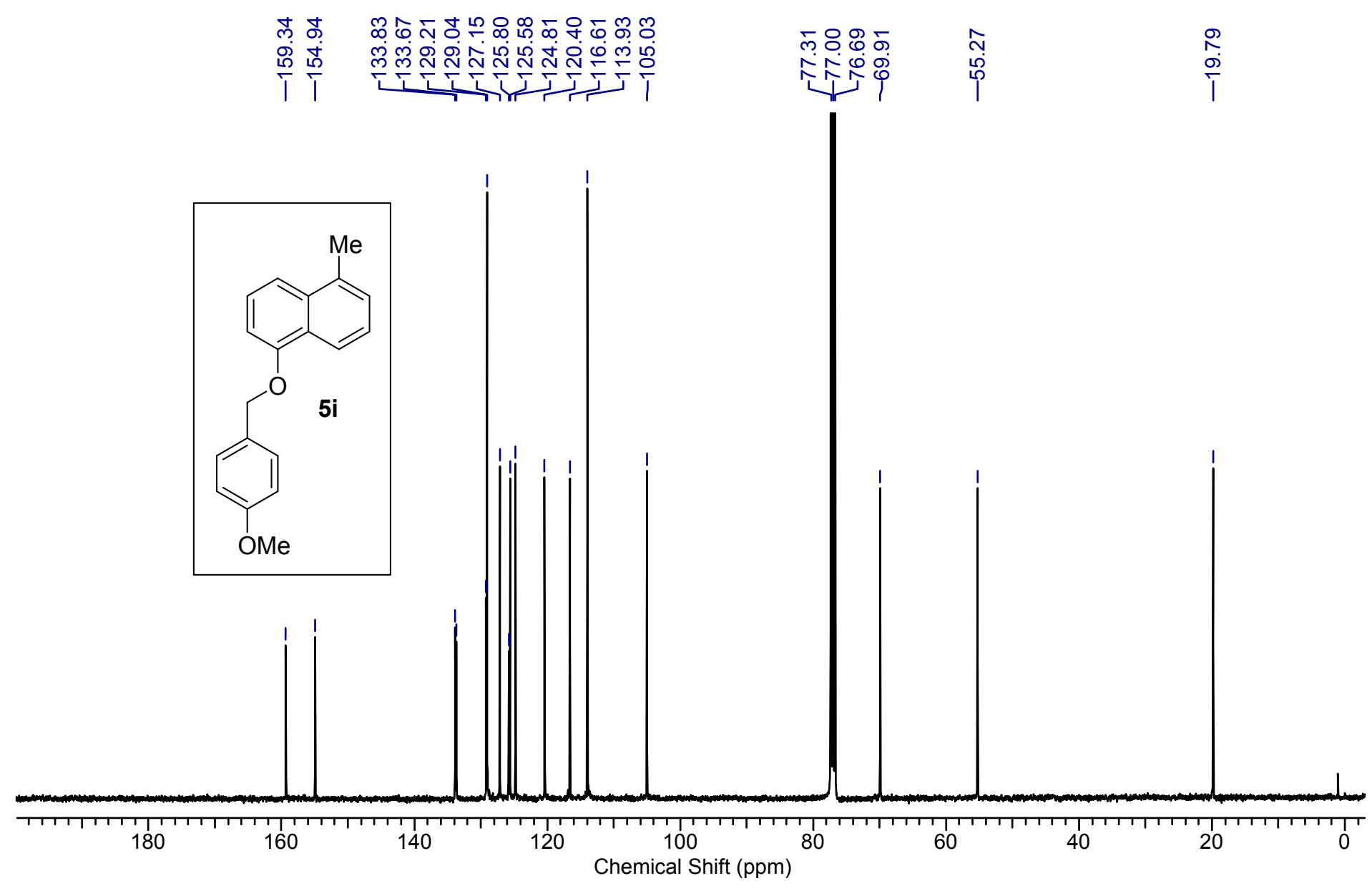

${ }^{13} \mathrm{C}\left\{{ }^{1} \mathrm{H}\right\}$ NMR of $5 \mathbf{i}\left(101 \mathrm{MHz}, \mathrm{CDCl}_{3}\right)$ 


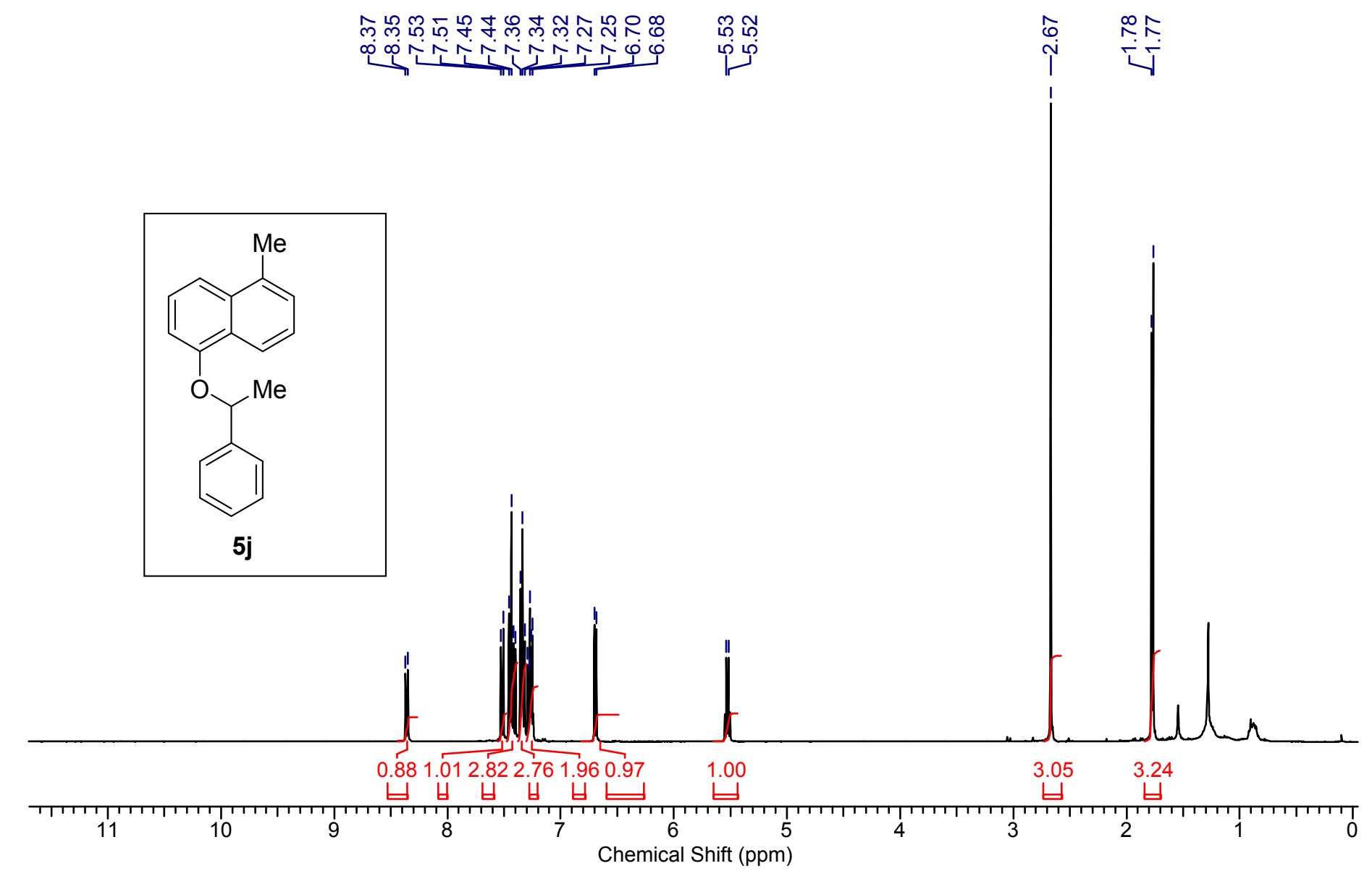

${ }^{1} \mathrm{H}$ NMR of $\mathbf{5 j}\left(400 \mathrm{MHz}, \mathrm{CDCl}_{3}\right)$ 


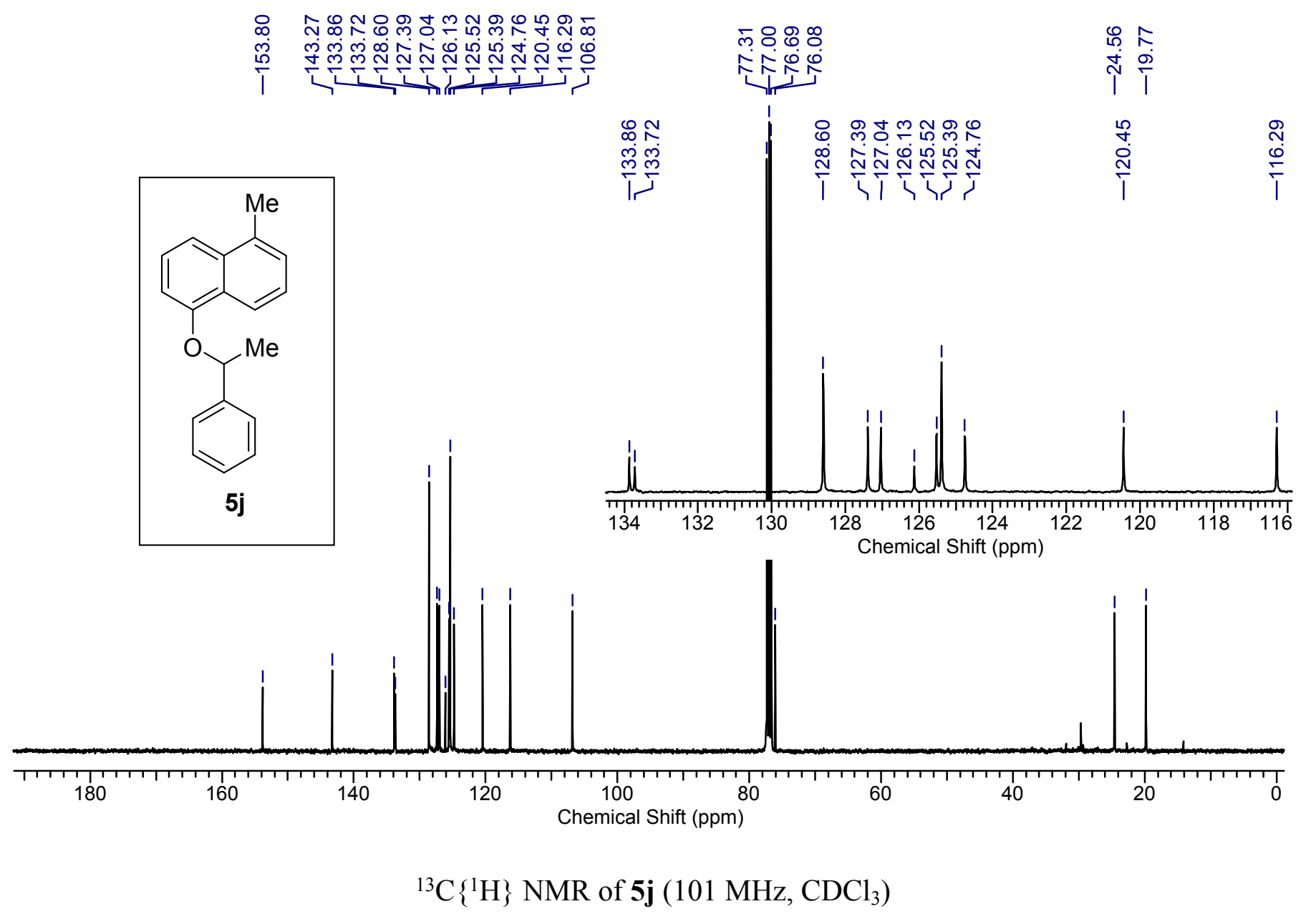




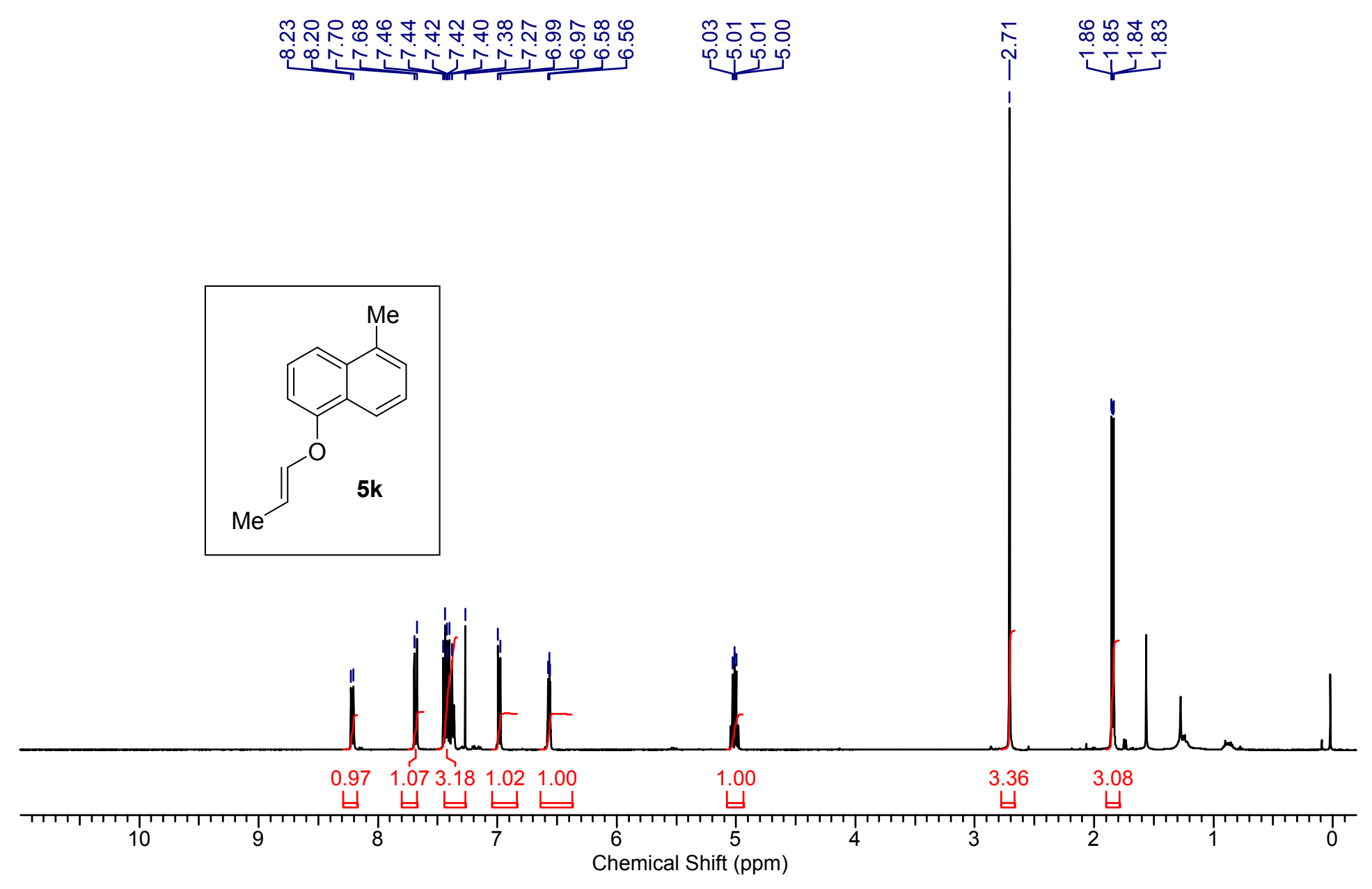

${ }^{1} \mathrm{H}$ NMR of $\mathbf{5 k}\left(400 \mathrm{MHz}, \mathrm{CDCl}_{3}\right)$ 


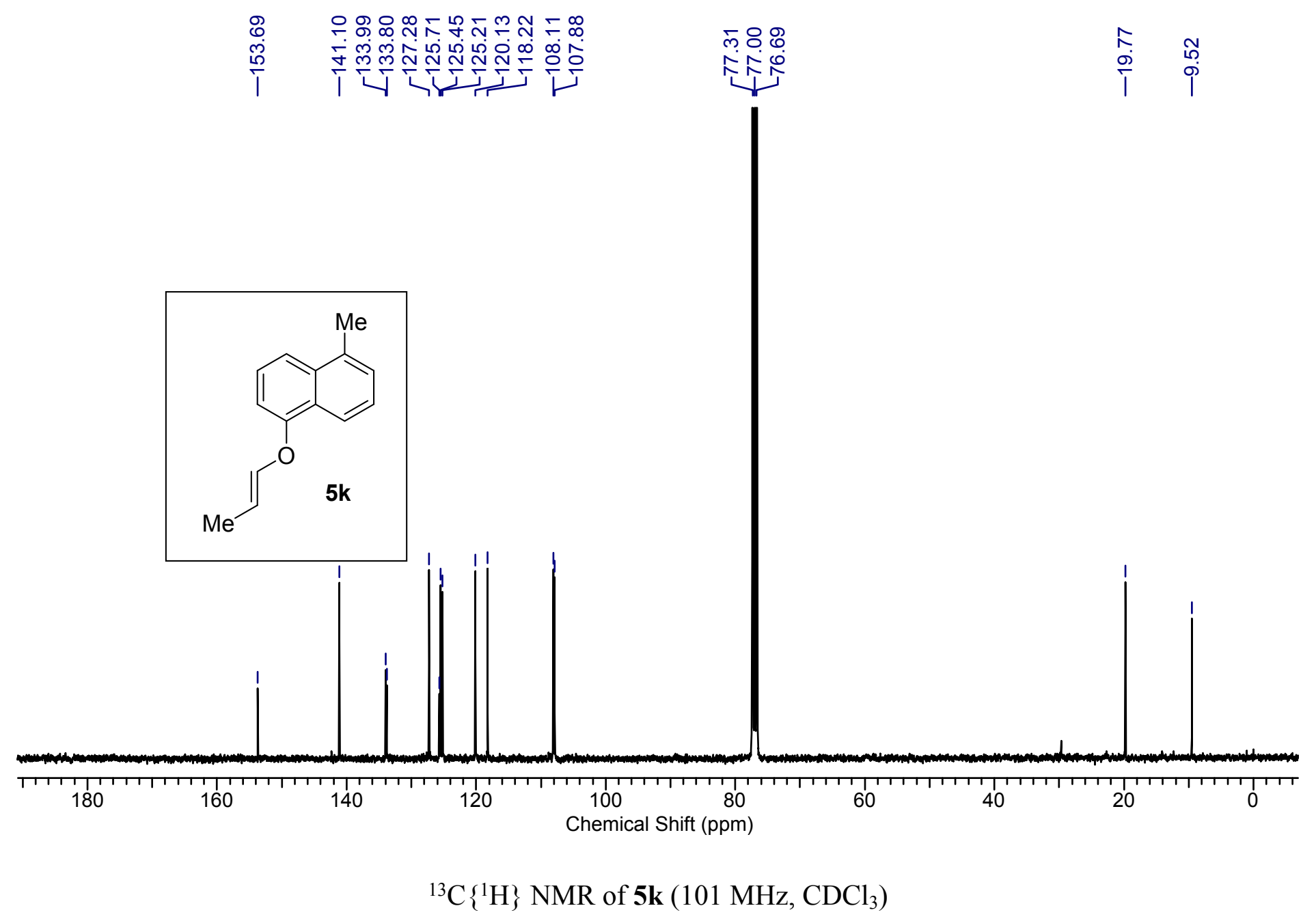




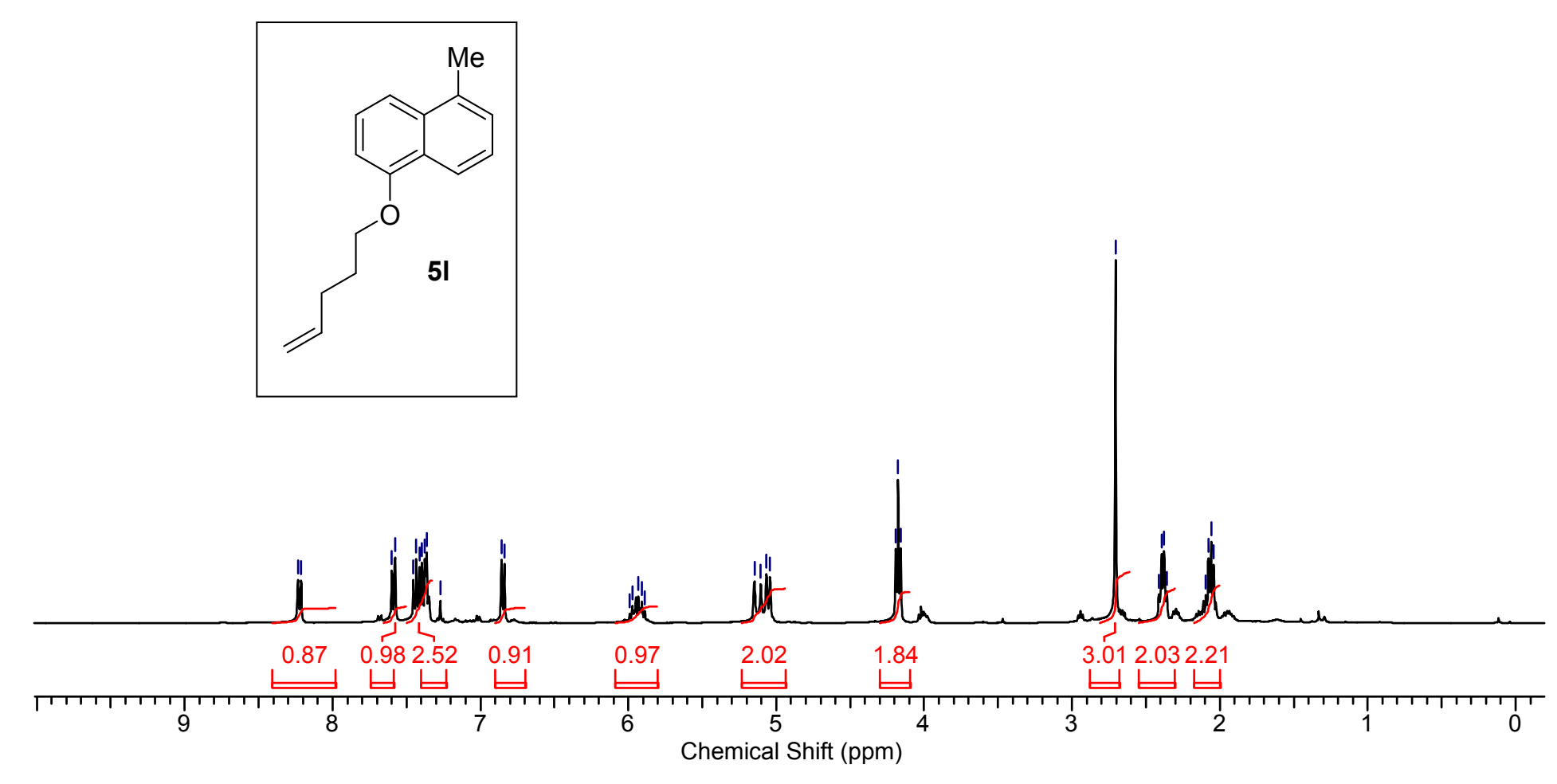

${ }^{1} \mathrm{H} \mathrm{NMR}$ of $\mathbf{5 l}\left(400 \mathrm{MHz}, \mathrm{CDCl}_{3}\right)$ 

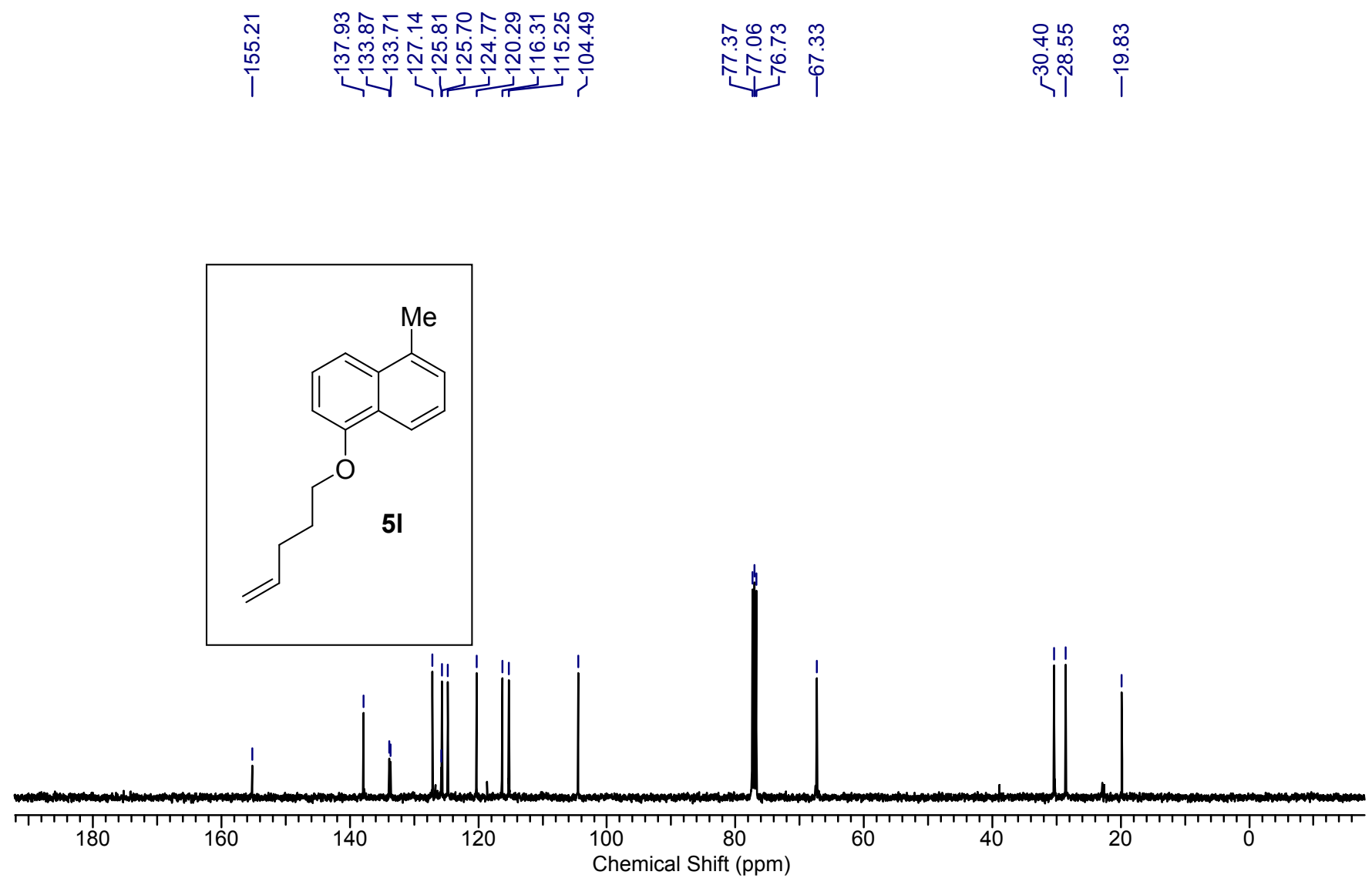

${ }^{13} \mathrm{C}\left\{{ }^{1} \mathrm{H}\right\}$ NMR of $5 \mathbf{l}\left(101 \mathrm{MHz}, \mathrm{CDCl}_{3}\right)$

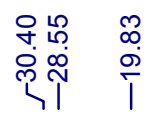

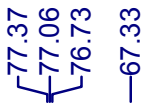

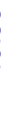

S87 


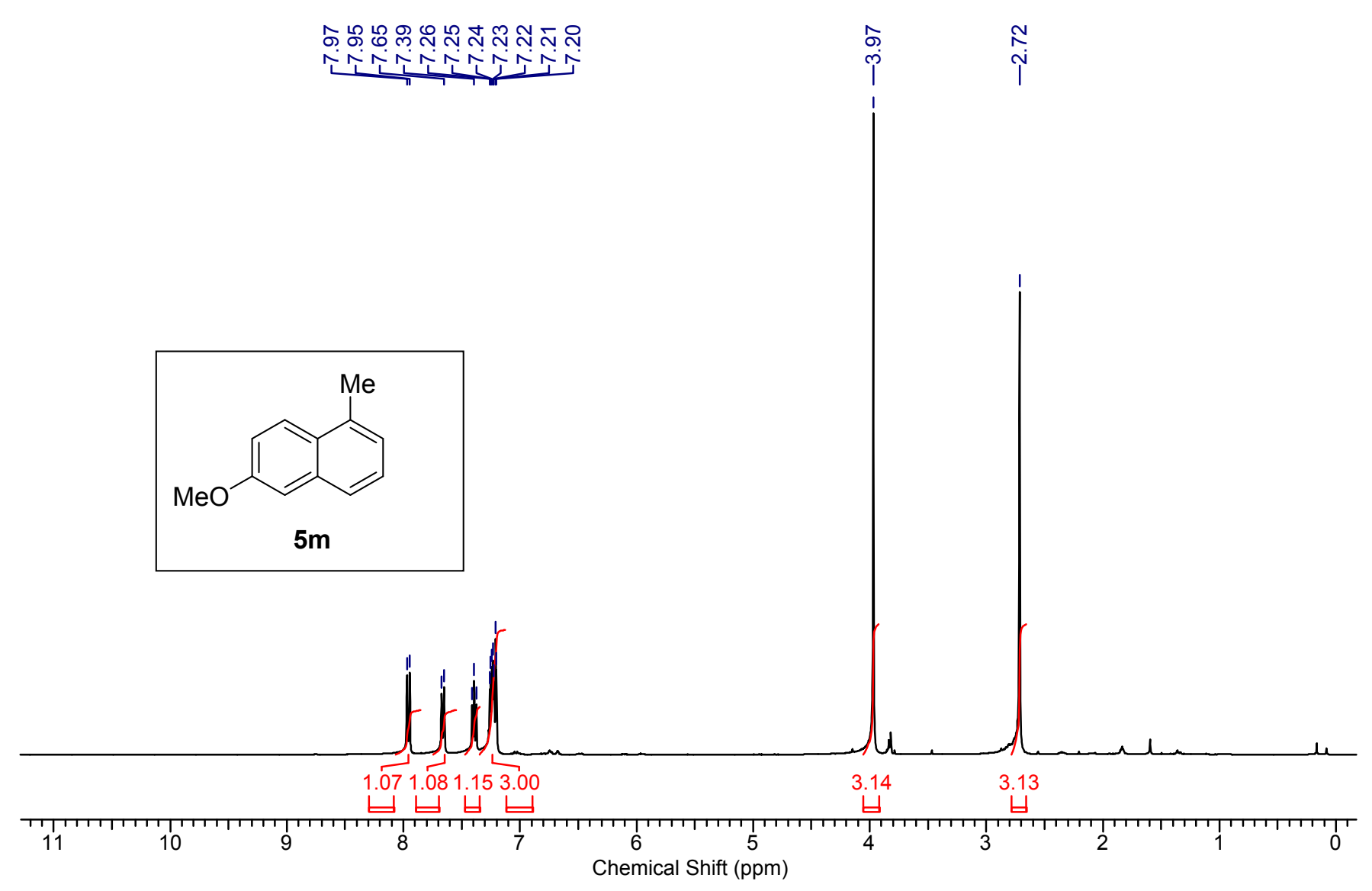

${ }^{1} \mathrm{H} \mathrm{NMR}$ of $\mathbf{5 m}\left(400 \mathrm{MHz}, \mathrm{CDCl}_{3}\right)$ 

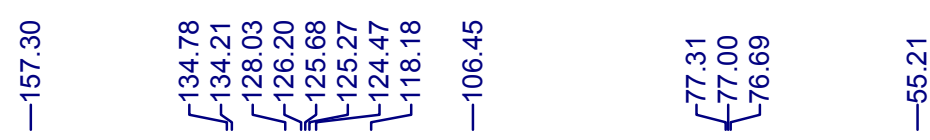

$\stackrel{m}{\stackrel{m}{\Gamma}}$

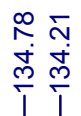

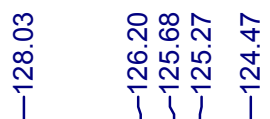
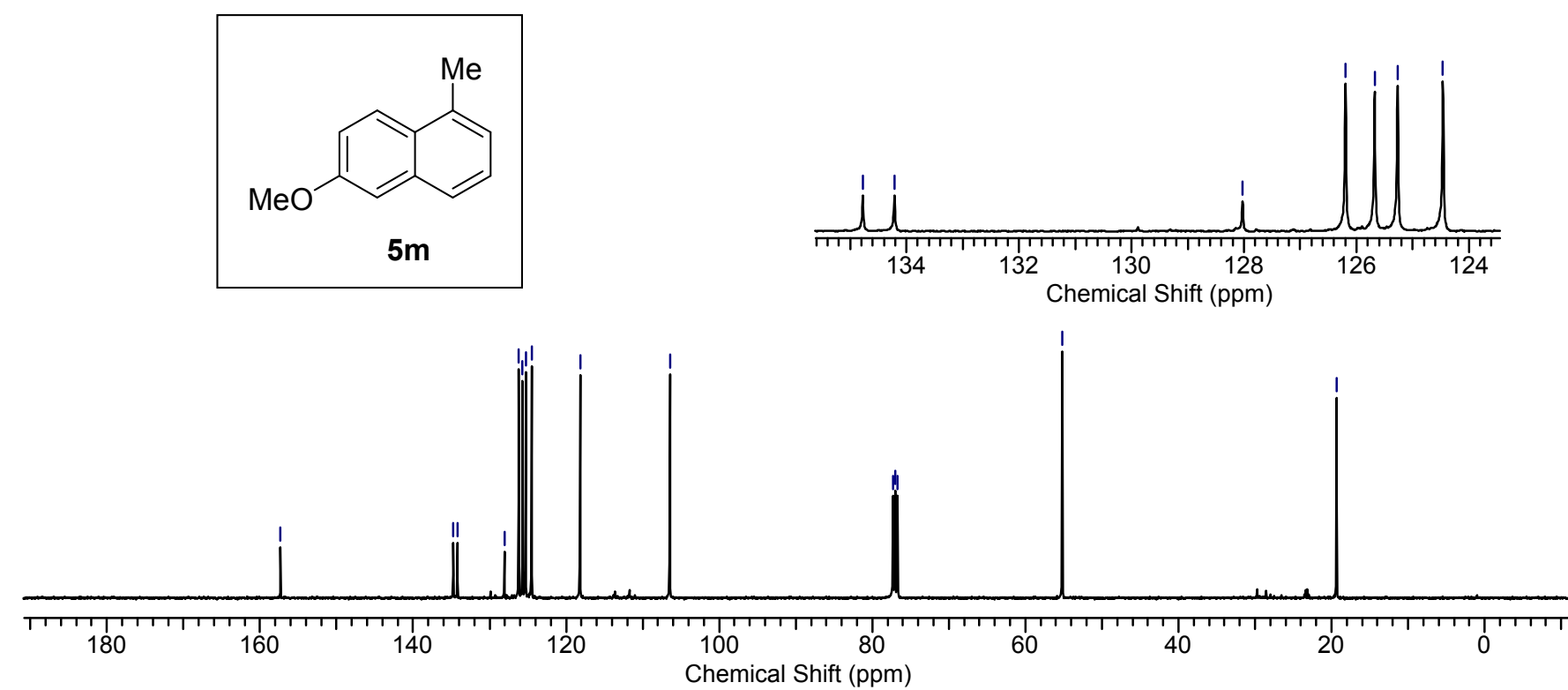

${ }^{13} \mathrm{C}\left\{{ }^{1} \mathrm{H}\right\}$ NMR of $\mathbf{5 m}\left(101 \mathrm{MHz}, \mathrm{CDCl}_{3}\right)$ 


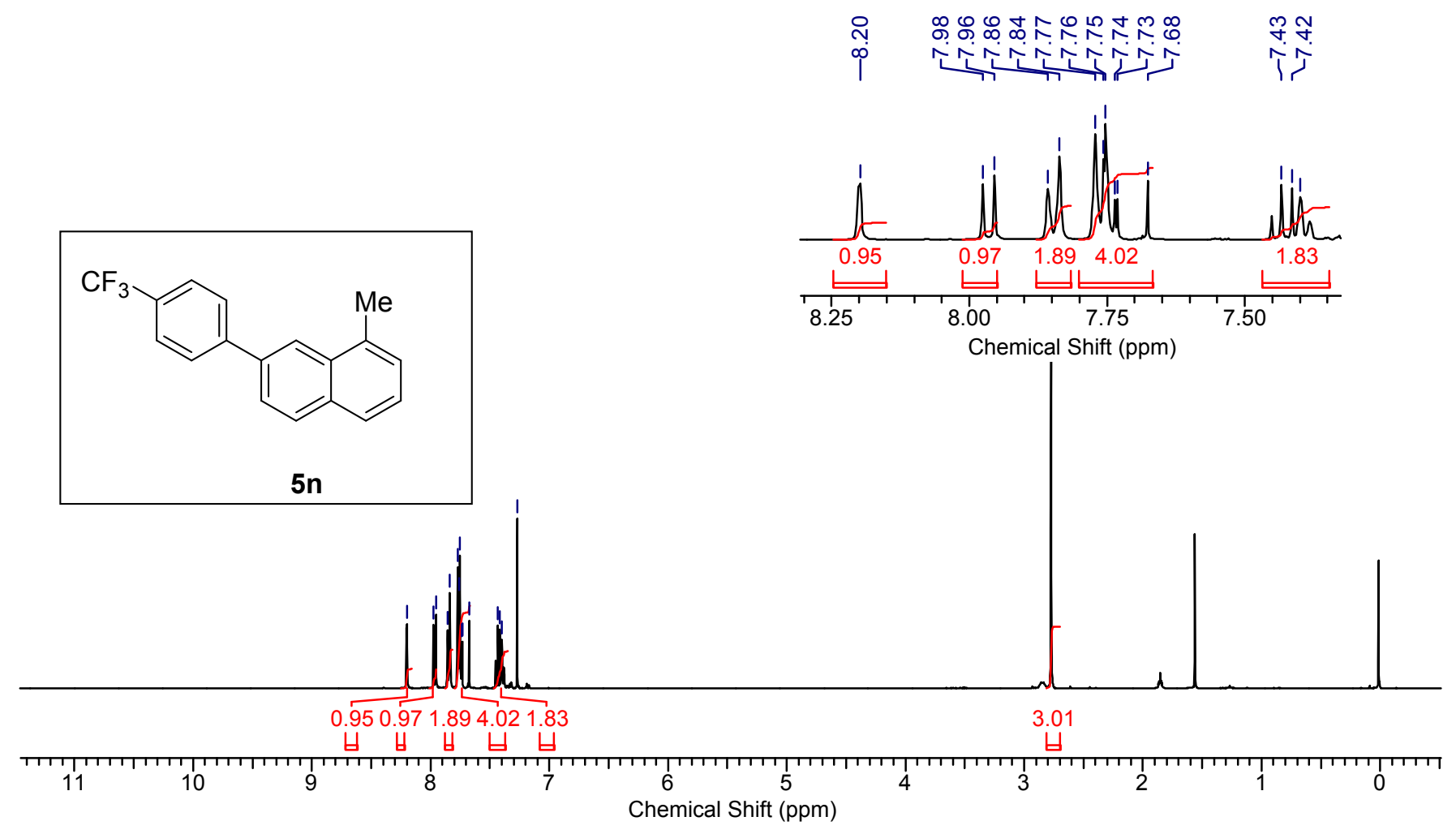

${ }^{1} \mathrm{H}$ NMR of $\mathbf{5 n}\left(400 \mathrm{MHz}, \mathrm{CDCl}_{3}\right)$ 


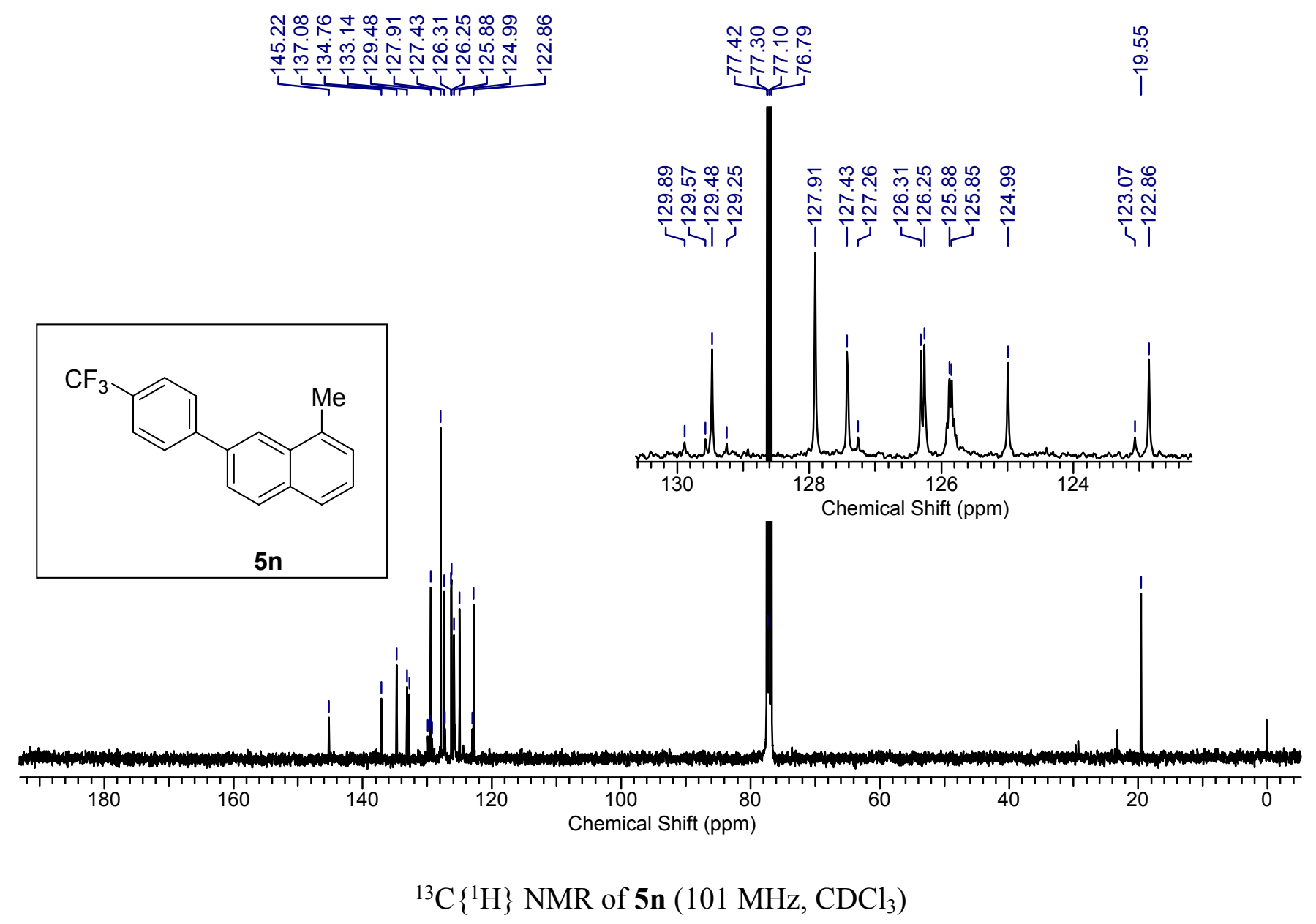



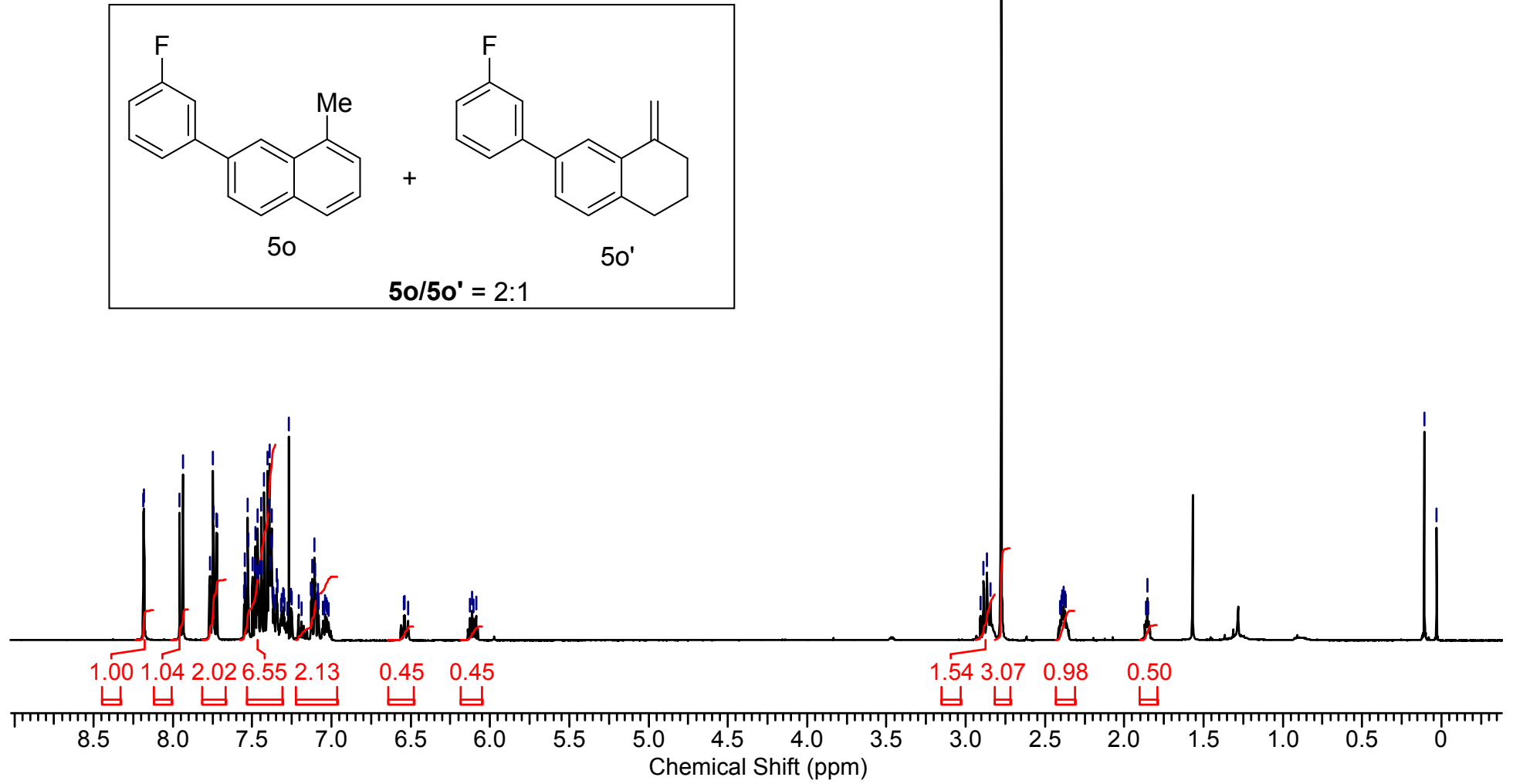

${ }^{1} \mathrm{H}$ NMR of 5 o $\left(400 \mathrm{MHz}, \mathrm{CDCl}_{3}\right)$ 
눙ㄷㅇ ๓

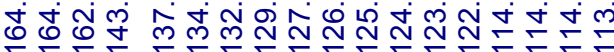

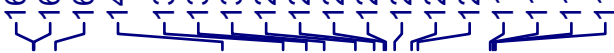

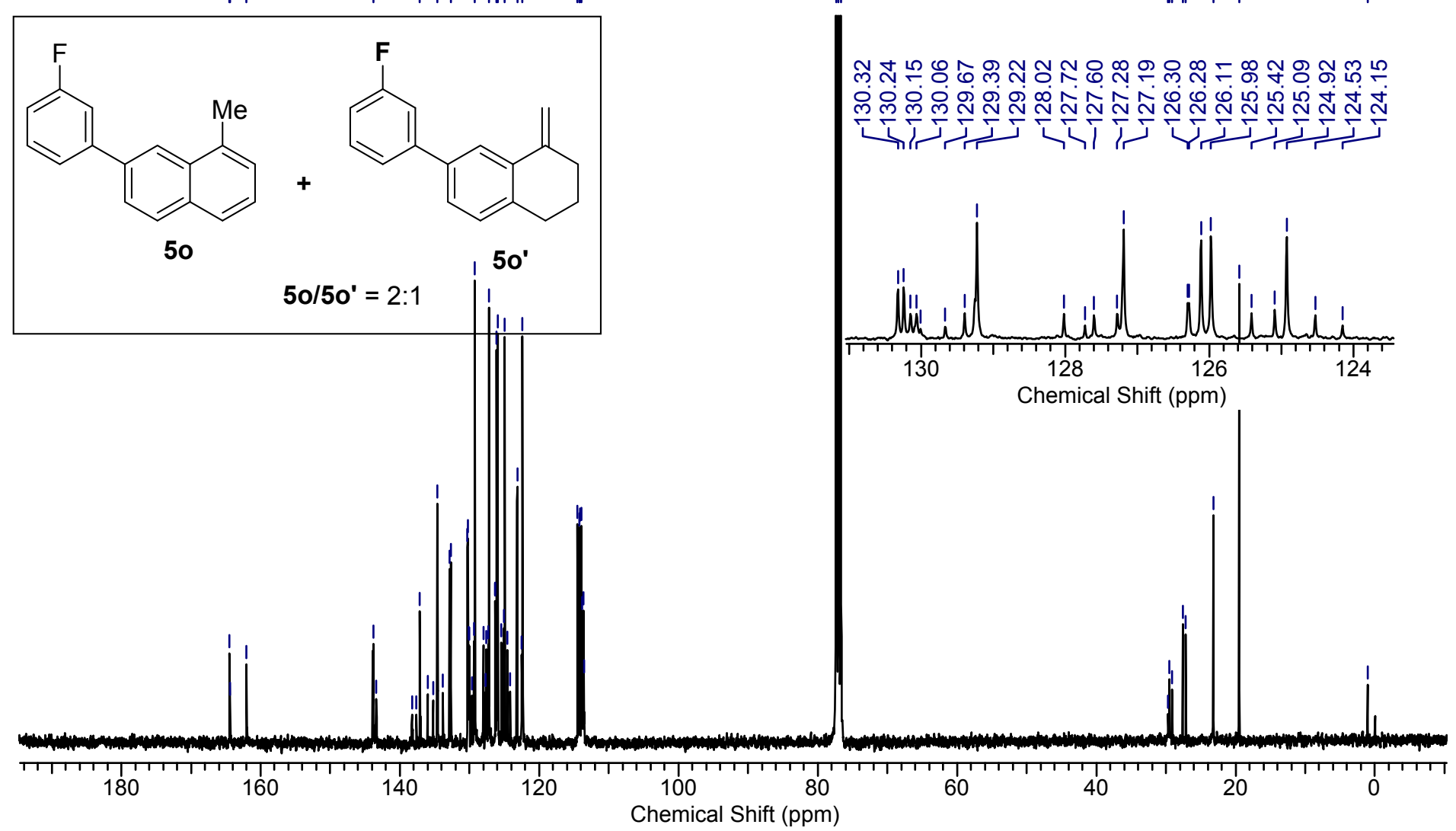

${ }^{13} \mathrm{C}\left\{{ }^{1} \mathrm{H}\right\}$ NMR of $50\left(101 \mathrm{MHz}, \mathrm{CDCl}_{3}\right)$ 


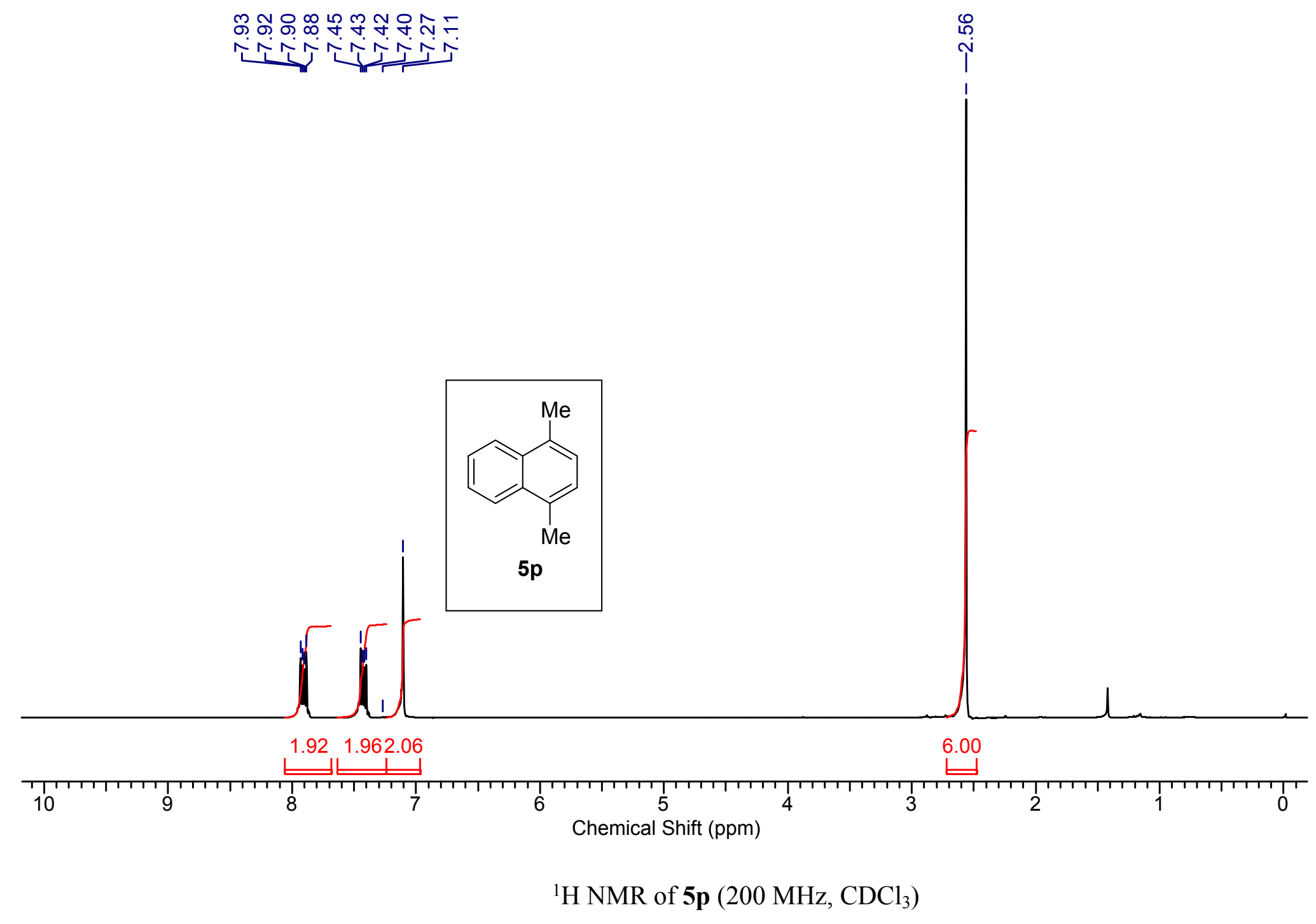




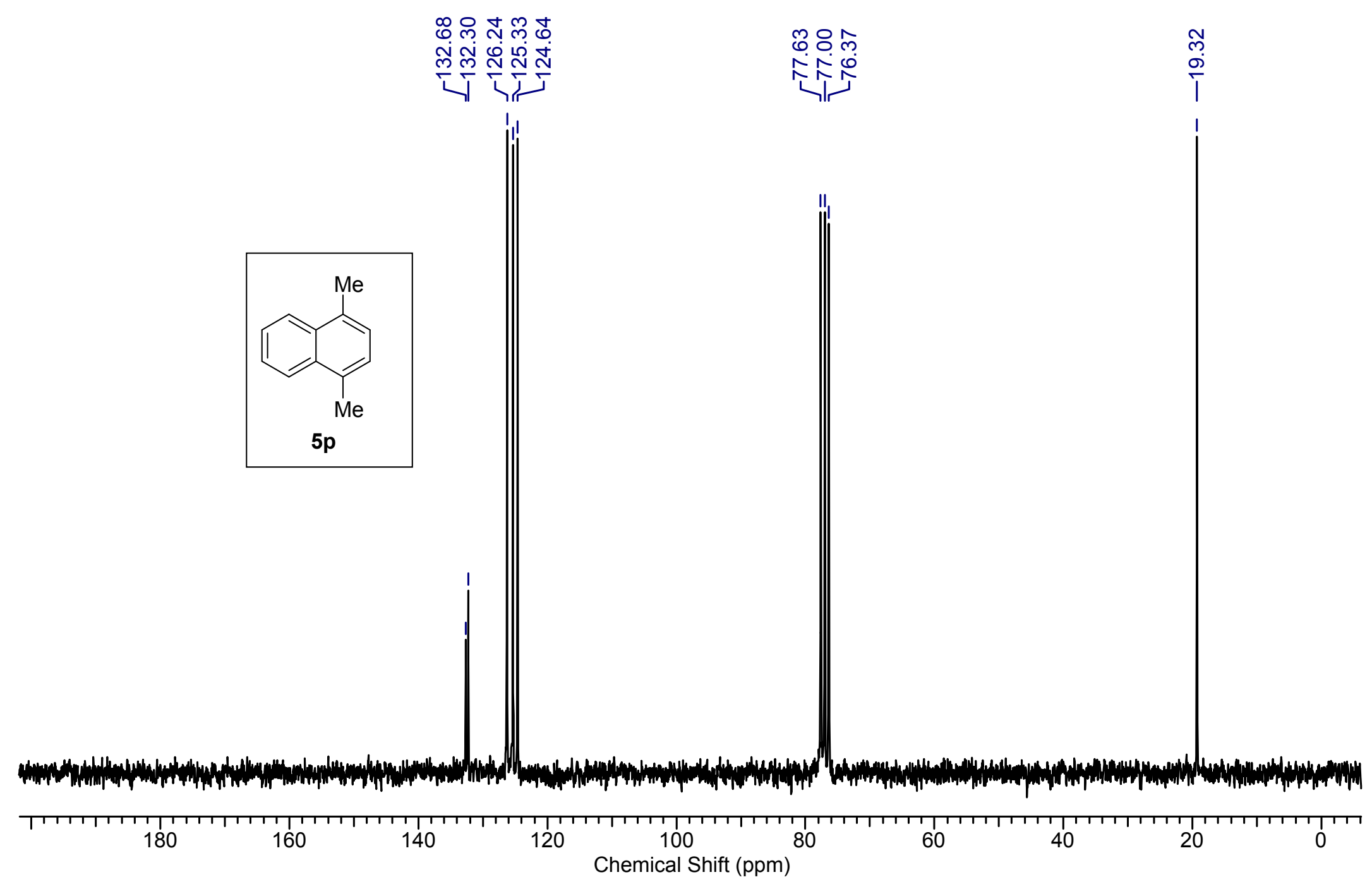

${ }^{13} \mathrm{C}\left\{{ }^{1} \mathrm{H}\right\} \mathrm{NMR}$ of $\mathbf{5 p}\left(50 \mathrm{MHz}, \mathrm{CDCl}_{3}\right)$ 